\title{
Spectral index-mass accretion rate correlation and evaluation of black hole masses in AGNs 3C 454.3 and M 87
}

\author{
Lev Titarchuk ${ }^{1,2,3}$, Elena Seifina ${ }^{3,4}$, Alexandre Chekhtman $^{5}$, and Indira Ocampo ${ }^{6}$ \\ 1 Dipartimento di Fisica, University di Ferrara, Via Saragat 1, 44122 Ferrara, Italy \\ e-mail: titarchuk@fe.infn.it \\ 2 Astro Space Center, Lebedev Physical Institute, Russian Academy of Science, Profsousnaya ul., 84/32, Moscow 117997, Russia \\ ${ }^{3}$ Lomonosov Moscow State University/Sternberg Astronomical Institute, Universitetsky Prospect 13, Moscow 119992, Russia \\ e-mail: seif@sai.msu.ru \\ 4 All-Russian Institute of Scientific and Technical Information, RAS, Usievich st. 20, Moscow 125190, Russia \\ 5 George Mason University, College of Science, 4400 University Drive, Fairfax, VA 22030, USA \\ e-mail: achekhtm@gmu.edu \\ ${ }^{6}$ Lomonosov Moscow State University/Physics Department, Vorobievy Hills, 1, Moscow 119234, Russia
}

Received 29 March 2019 / Accepted 31 October 2019

\begin{abstract}
We present the discovery of correlations between the X-ray spectral (photon) index and mass accretion rate observed in active galactic nuclei (AGNs) 3C 454.3 and M 87. We analyzed spectral transition episodes observed in these AGNs using Chandra, Swift, Suzaku, BeppoSAX, ASCA and RXTE data. We applied a scaling technique for a black hole (BH) mass evaluation which uses a correlation between the photon index and normalization of the seed (disk) component which is proportional to a mass accretion rate. We developed an analytical model that shows that the photon index of the $\mathrm{BH}$ emergent spectrum undergoes an evolution from lower to higher values depending on disk mass accretion rate. To estimate a BH mass in 3C 454.3 we consider extra-galactic SMBHs NGC 4051 and NGC 7469 as well as Galactic BHs Cygnus X-1 and GRO J1550-564 as reference sources for which distances, inclination angles are known and the $\mathrm{BH}$ masses are already evaluated. For M 87 on the other hand, we provide the $\mathrm{BH}$ mass scaling using extra-galactic sources (IMBHs: ESO 243-49 HLX-1 and M 101 ULX-1) and Galactic sources (stellar mass BHs: XTE J1550-564, 4U 1630-472, GRS 1915+105 and H 1743-322) as reference sources. Application of the scaling technique for the photon index-mass accretion rate correlation provides estimates of the $\mathrm{BH}$ masses in $3 \mathrm{C} 454.3$ and $\mathrm{M} 87$ to be about $3.4 \times 10^{9}$ and $5.6 \times 10^{7}$ solar masses, respectively. We also compared our scaling BH mass estimates with a recent BH mass estimate of $M_{87}=6.5 \times 10^{9} M_{\odot}$ made using the Event Horizon Telescope which gives an image at $1.3 \mathrm{~mm}$ and is based on the angular size of the "BH event horizon". Our BH mass estimate in M 87 is at least two orders of magnitude lower than that made by the EHT team.
\end{abstract}

Key words. accretion, accretion disks - black hole physics - galaxies: active

\section{Introduction}

Active galactic nuclei (AGNs) are ideal laboratories for studying the properties of matter in critical conditions. Especially interesting are radio-loud AGNs such as blazars and FR-I radio sources, which more often enter into active states and are accompanied by jet ejection events. Usually they are sources of multiwavelength radiation. It is also assumed that their energy is controlled by a supermassive black hole $(\mathrm{BH})$ at their center.

Blazars constitute a sub-class of radio-loud AGNs characterized by broadband nonthermal emission from radio to $\gamma$-rays. It is generally proposed that bipolar relativistic jets closely align to our line of sight. They exhibit rapid variability and a high degree of polarization. Blazars with only weak or entirely absent emission lines in the optical band are historically classified as BL Lacertae objects, while others are classified as flat-spectrum radio quasars (FSRQs, see Urry \& Padovani 1995).

Among the FSRQ class of blazars, 3C 454.3 (PKS 2251+158) is one of the brightest and most variable sources. This source is a well-known AGN and demonstrates significant variability in all energy bands Unwin et al. (1997): optical (Villata et al. 2009; Raiteri et al. 1998, 2011), radio (Volvach et al. 2008, 2010, 2011,
2013, 2017), X-rays (Raiteri et al. 2011; Abdo et al. 2010), and $\gamma$-rays (Bonning et al. 2009; Abdo et al. 2009).

In the optical range, the blazar 3C 454.3 shows a change of active states. For example, in 2006-2007 the source remained in a faint state (Raiteri et al. 2007). Then, from July 2007 the source began an active phase, which was detected several times in $\gamma$-rays by the AGILE satellite ${ }^{1}$. The source was monitored from the optical to the radio bands by the Whole Earth Blazar Telescope $^{2}$ (WEBT) and its GLAST-AGILE Support Program (GASP, Donnarumma et al. 2008). Vercellone et al. (2008, 2009, 2010), Raiteri et al. (2008a,b), Donnarumma et al. (2009), and Villata et al. (2009) published the results of these observations. GASP-WEBT (Global Aviation Safety Plan-Whole Earth Blazar Telescope) observations in 2009-2010 were performed in a number of observatories: Abastumani, Calar Alto data were acquired as part of the MAPCAT project ${ }^{3}$, Crimean, Galaxy View, Goddard (GRT- Gamma Ray Telescope), Kitt Peak [(MDM), MichiganDartmouth-MIT Observatory], Lowell (Perkins-Lowell Obser-

\footnotetext{
http://agile.iasf-roma.inaf.it/

http://www . oato.inaf.it/blazars/webt/

http://www.iaa.es/ iagudo/research/MAPCAT
} 
Table 1. Suzaku observations of 3C 454.3 and M 87 used in our analysis.

\begin{tabular}{llcccc}
\hline \hline Object & Number of set & Obs. ID & Start time (UT) & End time (UT) & MJD interval \\
\hline \multirow{2}{*}{ 3C 454.3 } & Sz1 & 703006010 & 2008 Nov. 22 09:18:32 & 2008 Nov. 23 16:31:19 & $54792.4-54793.7^{(1)}$ \\
& Sz2 & 705021010 & 2010 Nov. 25 01:11:14 & 2010 Nov. 26 00:20:19 & $55525.2-55526.8$ \\
& Sz3 & 904003010 & 2009 Dec. 9 02:40:43 & 2009 Dec. 9 23:31:24 & $54439.2-54439.8$ \\
& Sz4 & 902002010 & 2007 Dec. 5 04:57:55 & 2007 Dec. 6 02:40:14 & $55174.1-55175.4$ \\
\hline M87 & Sz & 801038010 & 2006 Nov. 29 22:02:65 & 2006 Dec. 2 03:02:24 & $54068.9-54071.1$ \\
\hline
\end{tabular}

References. ${ }^{(1)}$ Abdo et al. (2010).

vatory), Lulin, New Mexico Skies, Roque de los Muchachos (KVA-Kungliga Vetenskapsacademien), Sabadell, San Pedro Martir, St. Petersburg, Talmassons, Teide (BRT-the Bradford Robotic Telescope), Tijarafe, and Valle d'Aosta. Optical observations were also carried out by the UVOT instrument onboard the Swift satellite (Raiteri et al. 2011). This optical object with a magnitude of $V=16.1^{m}$ was also identified as a radio source and classified as a highly polarized quasar with a redshift of $z=0.859$ (Jackson \& Browne 1991).

A multi-wavelength study of the flaring behavior of $3 \mathrm{C} 454.3$ during 2005-2008 was carried out by Jorstad et al. (2010). These latter authors discussed the activity of this object in terms of a core-jet with a knot structure and suggested that the emergence of a superluminal knot from the core relates to a series of optical and high-energy outbursts and that the millimeter-wave core lies at the end of the jet acceleration and collimation zone.

Suzaku observations of 3C 454.3 in November 2008 analyzed by Abdo et al. (2010) confirmed the earlier suggestions by Raiteri et al. $(2011,2008 b)$ that there is a soft X-ray excess, which becomes stronger when the $\gamma$ - radiation in this source gets fainter. These authors interpret this excess as either a contribution of the high-energy tail of the synchrotron component or bulk-Compton radiation produced as a result of Comptonization of the disk UV photons due to the relativistic jet plasma.

Despite the 3C 454.3 study based on multi-wave observations, the question of the mass of the central engine in $3 \mathrm{C} 454.3$ still remains open. On the other hand, there is a problem with its extended structures, which are clearly visible on the radio images. We should also emphasize its strong variability which can be interpreted in the framework of the binary system model (see Volvach et al. 2017 and references there).

The mass of the super-massive black hole (SMBH) centered in $3 \mathrm{C} 454.3$ is estimated to be within a the range of $M=(0.5-1.5) \times 10^{9} M_{\odot}$ (Woo \& Urry 2002; Liu et al. 2006; Sbarrato et al. 2012). Gu et al. (2001) concluded that the quasar contains a binary system of BHs with masses of $0.5-4 \times$ $10^{9} M_{\odot}$, and Eddington luminosity that ranges from $6 \times 10^{46}$ to $5 \times 10^{47} \mathrm{erg} \mathrm{s}^{-1}$ (Khangulyan et al. 2013). The flow velocity of the relativistic jet probably ranges between $0.97 c$ and $0.999 c$ (Jorstad et al. 2005; Hovatta et al. 2009; Raiteri et al. 2011) and the angle to our line of sight is between $1^{\circ}$ and $6^{\circ}$ (Raiteri et al. 2011; Zamaninasab et al. 2013). Gupta et al. (2017) using optical data from the Steward observatory produced a more precise estimate of the $\mathrm{BH}$ mass of the central source using the broad Mg II line width and the continuum luminosity at $3000 \AA$ : $M_{\mathrm{BH}}=2.3 \pm 0.5 \times 10^{9} M_{\odot}$ (see also Vestergaard \& Osmer 2009).

It is known that $3 \mathrm{C} 454.3$ is an active source accompanied by jet ejection events that are associated with a sharp increase in radio emission. The jet is also detected in X-rays. It is important to note that the most prominent extragalactic X-ray jet is well observed in AGN M 87 [E0-type (Hubble 1926), FR-I class], where several bright components (blobs) of this one-sided jet can be separately observed. In particular, the blob HST-1 is located less than $1^{\prime \prime}$ from the nucleus of M 87, and is the closest of its kind to that position. It is interesting that the HST-1 flux sometimes becomes significantly higher than that of a nucleus component. Therefore, it is also important to separate the jet emission from the radiation of the nucleus.

In this paper we present our analysis of available Chandra, Swift, Suzaku, ASCA, BeppoSAX and RXTE observations of 3C 454.3 and M 87 and also reexamine previous conclusions on the $\mathrm{BH}$ nature of these AGNs. Furthermore, we find further indications to supermassive BHs in $3 \mathrm{C} 454.3$ and M 87. In Sect. 2 we present the list of observations used in our data analysis, and in Sect. 3 we provide details of the X-ray spectral analysis. We discuss the evolution of the X-ray spectral properties during the spectral state transitions and present the results of the scaling analysis to estimate a BH mass for these AGNs in Sect. 4. We present our final conclusions on the results in Sect. 5.

\section{Observations and data reduction}

Along with the long-term RXTE observations (1996-2010) described in Sect. 2.6, 3C 454.3 was also observed by Suzaku (2007-2010, see Sect. 2.1), Swift (2005-2010, see Sect. 2.4), and Chandra (2002, 2004, see Sect. 2.5). On the other hand, M 87 was observed by RXTE (1997-1998 and 2010), which is described in Sect. 2.6, BeppoSAX (1996; Sect. 2.3), Chandra (1996; Sect. 2.5), Suzaku (2006, see Sect. 2.1), and ASCA (1993, see Sect. 2.2). We extracted these data from the HEASARC archives and found that they cover a wide range of X-ray luminosities for both sources. We should recognize that the well-exposed Suzaku data are preferable for the determination of low-energy photoelectric absorption. Therefore, we start our study using Suzaku data of 3C 454.3. We also apply up-to-date SMART optical/near-infrared light curves that are available online ${ }^{4}$.

\subsection{Suzaku data}

Suzaku observed 3C 454.5 throughout the time period from 2008 to 2010 and investigated M 87 in 2006. Table 1 summarizes the start time, end time, and the MJD interval for each of these observations. One can see a description of the Suzaku experiment in Mitsuda et al. (2007). For observations obtained using a focal X-ray CCD camera (XIS, X-ray Imaging Spectrometer, Koyama et al. 2007, which is sensitive over the $0.3-12 \mathrm{keV}$ range, we used software of the Suzaku data processing pipeline (ver. 2.2.11.22). We carried out the data reduction and analysis following the standard procedure using the latest HEASOFT software package (version 6.25) and following the

4 WWW.astro.yale.edu/smarts/glast/home.php 
Table 2. Best-fit parameters of the Suzaku and Chandra spectra of 3C 454.3 in the $0.3-10 \mathrm{keV}$ range using the following five models: tbabs*power, tbabs*bbody, tbabs*(bbody+power), tbabs*bmc and tbabs*(bmc+power).

\begin{tabular}{|c|c|c|c|c|c|c|c|}
\hline & Parameter & 703006010 & 705021010 & 904003010 & 902002010 & 3127 & 4843 \\
\hline \multicolumn{8}{|c|}{ Model } \\
\hline tbabs & $N_{\mathrm{H}}\left(\times 10^{21} \mathrm{~cm}^{-2}\right)$ & $2.8 \pm 0.7$ & $3.28 \pm 0.06$ & $3.1 \pm 0.06$ & $3.2 \pm 0.4$ & $0.5 \pm 0.1$ & $0.8 \pm 0.4$ \\
\hline \multirow[t]{3}{*}{ Power-law } & $\Gamma_{\text {pow }}$ & $1.46 \pm 0.01$ & $1.48 \pm 0.01$ & $1.54 \pm 0.01$ & $1.56 \pm 0.01$ & $0.79 \pm 0.03$ & $1.29 \pm 0.02$ \\
\hline & $N_{\text {pow }}(\dagger)$ & $32.5 \pm 0.9$ & $130 \pm 2$ & $139 \pm 2$ & $139.6 \pm 0.1$ & $6.24 \pm 0.04$ & $12.72 \pm 0.03$ \\
\hline & $\chi_{\text {red }}^{2}$ (d.o.f.) & $1.23(1058)$ & $1.24(1797)$ & $1.06(1449)$ & $2.00(1643)$ & $1.28(282)$ & $1.36(441)$ \\
\hline tbabs & $N_{\mathrm{H}}\left(\times 10^{21} \mathrm{~cm}^{-2}\right)$ & $0.09 \pm 0.01$ & $0.08 \pm 0.03$ & $0.06 \pm 0.01$ & $0.08 \pm 0.2$ & $0.03 \pm 0.01$ & $0.01 \pm 0.01$ \\
\hline \multirow[t]{3}{*}{ Bbody } & $T_{\mathrm{BB}}(\mathrm{keV})$ & $1.00 \pm 0.01$ & $1.04 \pm 0.01$ & $1.10 \pm 0.01$ & $0.83 \pm 0.01$ & $1.09 \pm 0.02$ & $0.78 \pm 0.01$ \\
\hline & $N_{\mathrm{BB}}(\dagger)$ & $2.1 \pm 0.3$ & $8.68 \pm 0.08$ & $8.62 \pm 0.09$ & $7.57 \pm 0.05$ & $0.93 \pm 0.05$ & $0.9 \pm 0.1$ \\
\hline & $\chi_{\text {red }}^{2}$ (d.o.f.) & $4.76(1058)$ & $13.2(1797)$ & $6.96(1449)$ & $13.08(1643)$ & $2.59(282)$ & $6.7(441)$ \\
\hline tbabs & $N_{\mathrm{H}}\left(\times 10^{21} \mathrm{~cm}^{-2}\right)$ & $5.5 \pm 0.2$ & $5.4 \pm 0.1$ & $5.3 \pm 0.4$ & $5.8 \pm 0.6$ & $0.85 \pm 0.02$ & $0.9 \pm 0.3$ \\
\hline \multirow[t]{2}{*}{ Bbody } & $T_{\mathrm{BB}}(\mathrm{keV})$ & $2.46 \pm 0.09$ & $2.75 \pm 0.08$ & $2.16 \pm 0.05$ & $2.04 \pm 0.3$ & $1.9 \pm 0.6$ & $5.7 \pm 0.4$ \\
\hline & $N_{\mathrm{BB}}(\dagger)$ & $2.8 \pm 0.2$ & $10.6 \pm 0.5$ & $8.3 \pm 0.2$ & $8.58 \pm 0.2$ & $15 \pm 4$ & $170 \pm 10$ \\
\hline \multirow[t]{3}{*}{ Power-law } & $\Gamma_{\text {pow }}$ & $2.74 \pm 0.08$ & $2.43 \pm 0.05$ & $2.59 \pm 0.06$ & $2.85 \pm 0.05$ & $1.19 \pm 0.09$ & $1.44 \pm 0.05$ \\
\hline & $N_{\text {pow }}(\dagger)$ & $69 \pm 4$ & $240 \pm 8$ & $251 \pm 9$ & $284 \pm 8$ & $7.09 \pm 0.08$ & $0.13 \pm 0.05$ \\
\hline & $\chi_{\text {red }}^{2}$ (d.o.f.) & $0.95(1056)$ & $0.93(1795)$ & $0.86(1447)$ & $0.86(1641)$ & $1.18(280)$ & $1.27(439)$ \\
\hline tbabs & $N_{\mathrm{H}}\left(\times 10^{21} \mathrm{~cm}^{-2}\right)$ & $5.6 \pm 0.2$ & $7.4 \pm 0.5$ & $3.0 \pm 0.2$ & $3.9 \pm 0.2$ & $5.8 \pm 0.2$ & $5.0 \pm 0.1$ \\
\hline \multirow[t]{5}{*}{ bmc } & $\Gamma_{\mathrm{bmc}}$ & $1.51 \pm 0.03$ & $2.01 \pm 0.09$ & $1.99 \pm 0.02$ & $1.98 \pm 0.01$ & $1.11 \pm 0.03$ & $1.24 \pm 0.02$ \\
\hline & $T_{\mathrm{s}}(\mathrm{eV})$ & $180 \pm 50$ & $132 \pm 20$ & $120 \pm 9$ & $210 \pm 10$ & $260 \pm 20$ & $100 \pm 10$ \\
\hline & $\log A$ & $-0.14 \pm 0.01$ & $-0.35 \pm 0.01$ & $-0.80 \pm 0.01$ & $-0.41 \pm 0.04$ & $2 .{ }^{(\dagger \dagger)}$ & $2 .{ }^{(\dagger \dagger)}$ \\
\hline & $N_{\text {bmc }}(\dagger)$ & $2.51 \pm 0.09$ & $18.0 \pm 0.1$ & $20.3 \pm 0.1$ & $11.2 \pm 0.1$ & $1.1 \pm 0.1$ & $0.9 \pm 0.1$ \\
\hline & $\chi_{\text {red }}^{2}$ (d.o.f.) & $1.19(1056)$ & $1.06(1795)$ & $1.03(1447)$ & $1.02(1641)$ & $1.05(279)$ & $1.14(438)$ \\
\hline tbabs & $N_{\mathrm{H}}\left(\times 10^{21} \mathrm{~cm}^{-2}\right)$ & $5.6 \pm 0.2$ & $7.4 \pm 0.5$ & $3.0 \pm 0.2$ & $3.9 \pm 0.2$ & $0.2 \pm 0.1$ & $1.2 \pm 0.1$ \\
\hline \multirow[t]{4}{*}{ bmc } & $\Gamma_{\mathrm{bmc}}$ & $1.51 \pm 0.03$ & $2.01 \pm 0.09$ & $1.99 \pm 0.02$ & $1.98 \pm 0.01$ & $1.12 \pm 0.02$ & $1.36 \pm 0.06$ \\
\hline & $T_{\mathrm{s}}(\mathrm{eV})$ & $170 \pm 40$ & $130 \pm 10$ & $120 \pm 9$ & $200 \pm 10$ & $112 \pm 2$ & $104 \pm 2$ \\
\hline & $\log A$ & $-0.18 \pm 0.02$ & $-0.37 \pm 0.04$ & $-0.83 \pm 0.05$ & $-0.42 \pm 0.08$ & $0.22 \pm 0.09$ & $0.42 \pm 0.06$ \\
\hline & $N_{\mathrm{bmc}}(\dagger)$ & $2.51 \pm 0.09$ & $17 \pm 1$ & $20.2 \pm 0.3$ & $11.3 \pm 0.2$ & $0.76 \pm 0.09$ & $0.5 \pm 0.1$ \\
\hline \multirow[t]{3}{*}{ Power-law } & $\Gamma_{\text {pow }}$ & $2.83 \pm 0.07$ & $2.49 \pm 0.04$ & $2.61 \pm 0.02$ & $2.91 \pm 0.04$ & $-2.5 \pm 0.3$ & $-2.5 \pm 0.4$ \\
\hline & $N_{\text {pow }}(\dagger)$ & $70 \pm 3$ & $330 \pm 5$ & $139 \pm$ & $98 \pm 5$ & $0.01 \pm 0.01$ & $0.01 \pm 0.01$ \\
\hline & $\chi_{\text {red }}^{2}$ (d.o.f.) & $10.08(1054)$ & $1.02(1793)$ & $1.00(1445)$ & 1.03 (1639) & $1.09(278)$ & $1.2(437)$ \\
\hline
\end{tabular}

Notes. Errors are given at the $90 \%$ confidence level. ${ }^{(\dagger)}$ Normalization parameters of blackbody and bmc components are in units of $L_{35}^{\text {soft }} / d_{10}^{2} \mathrm{erg} \mathrm{s}^{-1} \mathrm{kpc}^{-2}$, where $L_{35}^{\text {soft }}$ is soft photon luminosity in units of $10^{35} \mathrm{erg} \mathrm{s}^{-1}, d_{10}$ is the distance to the source in units of $10 \mathrm{kpc}$. ${ }^{(\dagger \dagger)} \mathrm{When}$ parameter $\log (A) \geq 2$ we fixed this parameter at $\log (A)=2 . T_{\mathrm{BB}}$ and $T_{\mathrm{s}}$ are the temperatures of the blackbody and seed photon components, respectively (in $\mathrm{keV}$ and $\mathrm{eV}$ ). $\Gamma_{\text {pow }}$ and $\Gamma_{\mathrm{bmc}}$ are the indices of the power law and BMC, respectively.

Suzaku Data Reduction Guide ${ }^{5}$. The spectra of the sources were extracted using spatial regions centered on the nominal positions of $3 \mathrm{C} 454.3$ and $\mathrm{M} 87\left(\alpha=22^{\mathrm{h}} 53^{\mathrm{m}} 57 \mathrm{~s} 77, \delta=+16^{\circ} 08^{\prime} 53^{\prime \prime} 6\right.$, $\mathrm{J} 2000.0$ for $3 \mathrm{C} 454.3$ and $\alpha=12^{\mathrm{h}} 30^{\mathrm{m}} 49 \mathrm{~s} .42, \delta=+12^{\circ} 23^{\prime} 28^{\prime} .04$, J2000.0 for M 87), while a background was extracted from source-free regions which have a comparable size away from the source. The spectrum data were re-binned to provide at least 20 counts per spectral bin to validate the use of the $\chi^{2}$-statistic. We carried out spectral fitting applying XSPEC v12.10.1. The energy ranges around 1.75 and $2.23 \mathrm{keV}$ were not used for spectral fitting because of the known artificial structures in the XIS spectra around the Si and Au edges. Therefore, for spectral fits we chose the $0.3-10 \mathrm{keV}$ range for the XISs (excluding 1.75 and $2.23 \mathrm{keV}$ points).

The source count rate was variable by $40 \%$ for $3 \mathrm{C} 454.3$ and $60 \%$ for M 87 . We fitted the spectral data using a number of models (see Sect. 3.2.1) but the best-fits are obtained using the XSPEC BMC model (see Titarchuk et al. 1997) modified by absorption

\footnotetext{
5 http://heasarc.gsfc.nasa.gov/docs/suzaku/analysis/
}

by neutral gas of the solar composition (the XSPEC tbabs model). Using this model we found that the amplitude of X-ray flux variability changed by up to a factor of two. The results of the fits are given in Table 2 .

\subsection{ASCA data}

ASCA observed M 87 on June 7-8, 1993. Table 3 summarizes the start time, end time, and the MJD interval for this observation. A description of the ASCA experiment can be found in Tanaka et al. (1994). The solid imaging spectrometers (SIS) operated in Faint CCD-2 mode. The ASCA data were screened using the ftool ascascreen and the standard screening criteria. The spectrum for the source was extracted using spatial regions with a diameter of $3^{\prime}$ (for SISs) and 4' (for GISs) centered on the nominal position of M 87, while background was extracted from source-free regions of comparable size away from the source. The spectrum data were rebinned to provide at least 20 counts per spectral bin to validate the use of the $\chi^{2}$ statistic. The SIS and GIS data were fitted applying XSPEC in the 
Table 3. ASCA and BeppoSAX observations of M 87 used in our analysis.

\begin{tabular}{lcccc}
\hline \hline Satellite & Obs. ID & Start time (UT) & End time (UT) & MJD interval \\
\hline ASCA & 60033000 & 1993 Jun. 7 19:15:48 & 1993 Jun. 8 07:10:36 & 49145.8-49146.3 \\
BeppoSAX & 60010001 & 1996 Jul. 14 21:31:00 & 1996 Jul. 15 10:57:10 & 50278.8-50279.4 \\
\hline
\end{tabular}

Table 4. Best-fit parameters of the BeppoSAX and ASCA spectra of M 87 in the $0.3-10 \mathrm{keV}$ range.

\begin{tabular}{lcccccccccc}
\hline \hline ID & $\begin{array}{c}\alpha= \\
\Gamma-1\end{array}$ & $\begin{array}{c}T_{\mathrm{s}} \\
(\mathrm{eV})\end{array}$ & $\log (A)$ & $N_{\mathrm{bmc}}{ }^{(\dagger)}$ & $\begin{array}{c}E_{\mathrm{cut}} \\
(\mathrm{keV})\end{array}$ & $\begin{array}{c}E_{\text {fold }} \\
(\mathrm{keV})\end{array}$ & $\begin{array}{c}E_{\mathrm{Fe}} \\
(\mathrm{keV})\end{array}$ & $\begin{array}{c}\sigma_{\mathrm{Fe}} \\
(\mathrm{keV})\end{array}$ & $\begin{array}{c}N_{\mathrm{Fe}}{ }^{(\dagger)} \\
(\text { d.o.f. })\end{array}$ \\
\hline 6001000 & $1.9 \pm 0.1$ & $140 \pm 20$ & $0.72 \pm 0.6$ & $1.1 \pm 0.1$ & $3.6 \pm 0.1$ & $9.8 \pm 0.4$ & $6.5 \pm 0.1$ & $0.7 \pm 0.1$ & $0.10 \pm 0.01$ & $0.95(40)$ \\
60033000 & $1.4 \pm 0.1$ & $139 \pm 30$ & $2.00^{(\dagger \dagger)}$ & $0.59 \pm 0.08$ & $3.5 \pm 0.1$ & $9.9 \pm 0.6$ & $6.4 \pm 0.1$ & $0.6 \pm 0.1$ & $0.09 \pm 0.01$ & $0.95(40)$ \\
\hline
\end{tabular}

Notes. Errors are given at the $90 \%$ confidence level. The spectral model is tbabs*(bmc+N*Gauss); where $N_{\mathrm{H}}$ is fixed at a value $5.0 \times 10^{21} \mathrm{~cm}^{-2}$ (see Sect. 3.2.1). ${ }^{(\dagger)}$ For normalization parameter $N_{\mathrm{BMC}}=L_{37} / d_{10}^{2}$ where $L_{37}$ is the source luminosity in units of $10^{37} \mathrm{erg} \mathrm{s}^{-1}$ and $d_{10}$ is the distance to the source in units of $10 \mathrm{kpc} .{ }^{(\dagger)}$ When parameter $\log \left(A_{2}\right) \gg 1$, this parameter is fixed at 2.0 (see comments in the text).

Table 5. Swift observations of 3C 454.3 used in our analysis.

\begin{tabular}{llll}
\hline \hline Obs. ID & Start time (UT) & End time (UT) & MJD interval \\
\hline $00030024(001,002)$ & 2005 May 11 & 2005 May 19 & $53501-53509$ \\
$00031018(001,003-008)$ & 2007 Nov. 15 & 2007 Dec. 15 & $54419-54449$ \\
$00031216(001-011,013-016,018-027,029-044,046-063)$ & 2008 May 27 & 2008 Oct. 2 & $54613-54741$ \\
$00031493(003,004)$ & 2009 Sep. 18 & 2009 Sep. 19 & $55092-55093$ \\
$00035030(001,003,005-010,013-016,027-067)$ & 2005 Apr. 24 & 2009 Sep. 16 & $53484-55090$ \\
$00035030(113,114,175,178,180-189,190-193,195-204)$ & 2010 Nov. 3 & 2011 Jun. 17 & $55503-55729$ \\
$00090023(001-008)$ & 2008 Sep. 9 2009 Jun. 1 13 20:37:05 55056-55087 \\
$00090081(001,002)$ & 2009 Aug. 13 12:56:32 & 2009 Sep. 13 \\
\hline
\end{tabular}

energy ranges of $0.6-10 \mathrm{keV}$ and $0.8-10 \mathrm{keV}$, where the spectral responses are best known.

\subsection{BeppoSAX data}

We used BeppoSAX data of M 87 carried out on June 14-15, 1996. In Table 4 we show the log of the BeppoSAX observation analyzed in this paper. Generally, broadband energy spectra of M87 Lac were obtained combining data from three BeppoSAX narrow-field instruments (NFIs): the Low Energy Concentrator Spectrometer (LECS; Parmar et al. 1997) for the 0.3-4 keV range, the Medium Energy Concentrator Spectrometer (MECS; Boella et al. 1997) for the 1.8-10 keV range, and the Phoswich Detection System (PDS; Frontera et al. 1997) for the 15-200 keV range. The SAXDAS data analysis package is used for the data processing. We performed a spectral analysis for each of the instruments in a corresponding energy range within which a response matrix is well specified. The LECS data have been renormalized to match the MECS data. Relative normalizations of the NFIs were treated as free parameters in the model fits, except for the MECS normalization that was fixed at unity. While the source is bright and background is low and stable, we checked its uniform distribution across the detectors. Furthermore, we extracted a light curve from a source-free region far from source and stated that background was not varying during the whole observation. Additionally, spectra were rebinned in accordance with the energy resolution of the instruments using rebinning template files in GRPPHA of $\mathrm{XSPEC}^{6}$ to obtain a

\footnotetext{
6 http://heasarc.gsfc.nasa.gov/FTP/sax/cal/responses/ grouping
}

better signal-to-noise ratio. Systematic uncertainties of $1 \%$ were applied to these analyzed spectra.

\subsection{Swift data}

We used the Swift observation of 3C 454.3 and M 87 carried out from 2005 to 2011 (Table 5). The Swift/XRT data were taken in Photon-Counting (PC) and Windowed Timing (WT) modes. The PC mode is characterized by high sensitivity but can be affected by the photon pile-up effect when the count rate is higher than $\sim 0.5 \mathrm{cts} \mathrm{s}^{-1}$, while the WT mode does not suffer from a photon pile-up effect up to $\sim 200 \mathrm{~s}^{-1}$. Data were processed using the HEASOFT v6.14, the tool XRTPIPELINE v0.12.84, and the calibration files (CALDB version 4.1). The ancillary response files were created using XRTMKARF v0.6.0 and exposure maps generated by XRTEXPOMAP v0.2.7. We selected source events for which the grades are accumulated in the range $0-12$ and used default screening parameters to produce level 2 cleaned event files. To avoid photon pile-up effect during high-count-rate events, we extract the spectral data obtained in the PC mode in an annular region with inner and outer radii of 6 and 20 pixels, respectively (Vaughan et al. 2006). The background was estimated in a nearby source-free circular region of 50 pixels in radius. For the WT data mode we extracted spectra in a $40 \times 20$ pixel rectangular region centered on the nominal position of $3 \mathrm{C} 454.3$. The background was estimated in a nearby $50 \times 20$ pixel source-free rectangular region. Spectra were re-binned to include at least 20 photons in each energy channel in order to use $\chi^{2}$-statistics. We fitted the spectrum using the response file SWXPC0TO12S6_20010101v012.RMF. We also 
Table 6. Chandra observations of 3C 454.3 and M 87.

\begin{tabular}{lcccc}
\hline \hline Object & Obs. ID & Start time (UT) & MJD & Exposure time (s) \\
\hline \multirow{2}{*}{ 3C 454.3 } & $3127^{(1)}$ & $2002-11-0621: 26: 22$ & 52584 & 4930 \\
& $4843^{(1)}$ & $2004-10-2905: 01: 50$ & 53221 & 18261 \\
\hline M 87 & $11514^{(2),(3)}$ & $2010-04-1520: 32: 42$ & 55301 & 4528 \\
& $18783^{(2),(3)}$ & $2016-04-2008: 32: 11$ & 55301 & 36114 \\
& $21075^{(2),(3)}$ & $2018-04-2203: 31: 16$ & 58230 & 9132 \\
\hline
\end{tabular}

References. ${ }^{(1)}$ Gupta et al. (2017); ${ }^{(2)}$ Abramowski et al. (2012); ${ }^{(3)}$ Harris et al. (2009).

Table 7. RXTE observations of $3 \mathrm{C} 454.3$ and M 87.

\begin{tabular}{lcccc}
\hline \hline Object & Number of set & Dates, MJD & RXTE proposal ID & Dates UT \\
\hline \multirow{2}{*}{ 3C 454.3 } & $R 1$ & $50181-50358$ & $10360^{(1)}$ & Apr. 8-Oct. 29, 1996 \\
& $R 2$ & $50389-50802$ & $20346^{(1)}$ & Nov. 2, 1996-Dec. 20, 1997 \\
& $R 3$ & $50816-50986$ & $30264^{(1)}$ & Jan. 3-Jun. 22, 1998 \\
& $R 4$ & $54309-54636$ & $93150^{(1)}$ & July 28, 2007-June 19, 2008 \\
& $R 5$ & $55170-55182$ & $94150^{(1)}$ & Dec. 5-Dec. 17, 2009 \\
& $R 6$ & $55293-55297$ & $95149^{(1)}$ & Apr. 7-Apr. 11, 2010 \\
\hline M 87 & $R_{1}^{\mathrm{m} 87}$ & $50812-50846$ & $30216^{(2)}$ & Dec. 30, 1997-Feb. 2, 1998 \\
& $R_{2}^{\mathrm{m} 87}$ & $55296-55301$ & $95145^{(2)}$ & Apr. 10-Apr. 15, 2010 \\
\hline
\end{tabular}

References. ${ }^{(1)}$ Rivers et al. (2013); ${ }^{(2)}$ Jorstad et al. (2010).

used the online XRT data product generator ${ }^{7}$ to obtain the image, light curves, and spectra (including background and ancillary response files; Evans et al. 2007, 2009).

\subsection{Chandra data}

The object 3C 454.3 was also observed by Chandra in 2002 and 2004, and M 87 was extensively investigated by Chandra: here we apply observations of 2010, 2016, and 2018. The used log of Chandra observations is presented in Table 6. We extracted spectra from the ACIS-S detector via the standard pipeline CIAO v4.5 package and calibration database CALDB 2.27. In Fig. 3 we demonstrate the Chandra/ASIS $(0.3-10 \mathrm{keV})$ image of the 3C 454.3 field of view on November 6, 2002, with the exposure of $4929 \mathrm{~s}(\mathrm{MJD}=52584)$. We also identified intervals of high background level to exclude all high-background events. The Chandra spectra were produced and modeled over the $0.3-7.0 \mathrm{keV}$ energy range.

\subsection{RXTE data}

For our analysis, we also applied publicly available data of the RXTE (Bradt et al. 1993) from October 1996 to December 2009 (for 3C454.3) and from December 1997 to February 1998 as well as April 2010 (for M 87). These data consist of 160 observations related to the different spectral states of the source. For data processing we utilized standard tasks of the LHEASOFT/FTOOLS 6.25 software package. Spectral analysis was implemented using PCA Standard 2 mode data, collected in the $3-22 \mathrm{keV}$ range. The standard dead-time correction procedure has been applied to these data. The data are available through the GSFC public archive ${ }^{8}$. We modeled the RXTE spectra using XSPEC astrophysical fitting software and implemented

\footnotetext{
7 http://www.swift.ac.uk/user_objects/

8 http://heasarc.gsfc.nasa.gov
}

a systematic uncertainty of $0.5 \%$ to all analyzed spectra. In Table 7 we present a list of the groups of observations which covers the complete sample of the state evolution of the source. In Fig. 1 we show an evolution of ASM/RXTE count rate $(2-12 \mathrm{keV}$, top $)$, radio $(8 \mathrm{GHz}$, middle $)$, and $\gamma$-ray radiation $(0.1-$ $300 \mathrm{GeV}$, bottom) during 1996-2012 observations of 3C 454.3. Vertical arrows (at top of the panels) indicate temporal distribution of the RXTE (blue) and Suzaku (green) data sets listed in Tables 1-7.

\subsection{Fermi/LAT data}

$\gamma$-ray data observed with Fermi/LAT were obtained from MJD 54690 to $58446^{9}$. The $\gamma$-ray source 1FGL2253.9+1608 was positionally identified with 3C 454.3 (Sasada et al. 2010). Each point on the $\gamma$-ray light curve (Fig. 2) corresponds to a daily averaged photon flux integrated over energies from $100 \mathrm{MeV}$ to $300 \mathrm{GeV}$. This plot shows that there is no correlation between $\gamma$ and X-ray flashes in 3C 454.3 observed in 2010-2012 for two bands: $2-12 \mathrm{keV}$ (top) and $0.1-300 \mathrm{GeV}$ (bottom).

\section{Results}

\subsection{Images and light curves of $3 C 454.3$ and $M 87$}

For a deep image analysis, we used the Chandra images with sub-arcsecond-resolution data quality provided by ACISS onboard Chandra. The Chandra/ACIS-S $(0.2-8 \mathrm{keV})$ image obtained during observations of 3C 454.3 on November 6, 2002, (with exposure of $5 \mathrm{ks}$, ObsID = 3127) is shown in Fig. 3. For each observation, we extracted the source spectrum from a circular region of $1.25^{\prime \prime}$ radius centered on the source position of 3C $454.3\left(\alpha=22^{\mathrm{h}} 53^{\mathrm{m}} 57 \mathrm{~s} .7, \delta=16^{\circ} 08^{\prime} 53 ! 6, \mathrm{~J} 2000.0\right.$ ) (see details in Abdo et al. 2010) and $0.75^{\prime \prime}$ radius circular region

\footnotetext{
9 http://fermi.gsfc.nasa.gov/ssc/data/analysis/ scitools/extract_latdata.html
} 


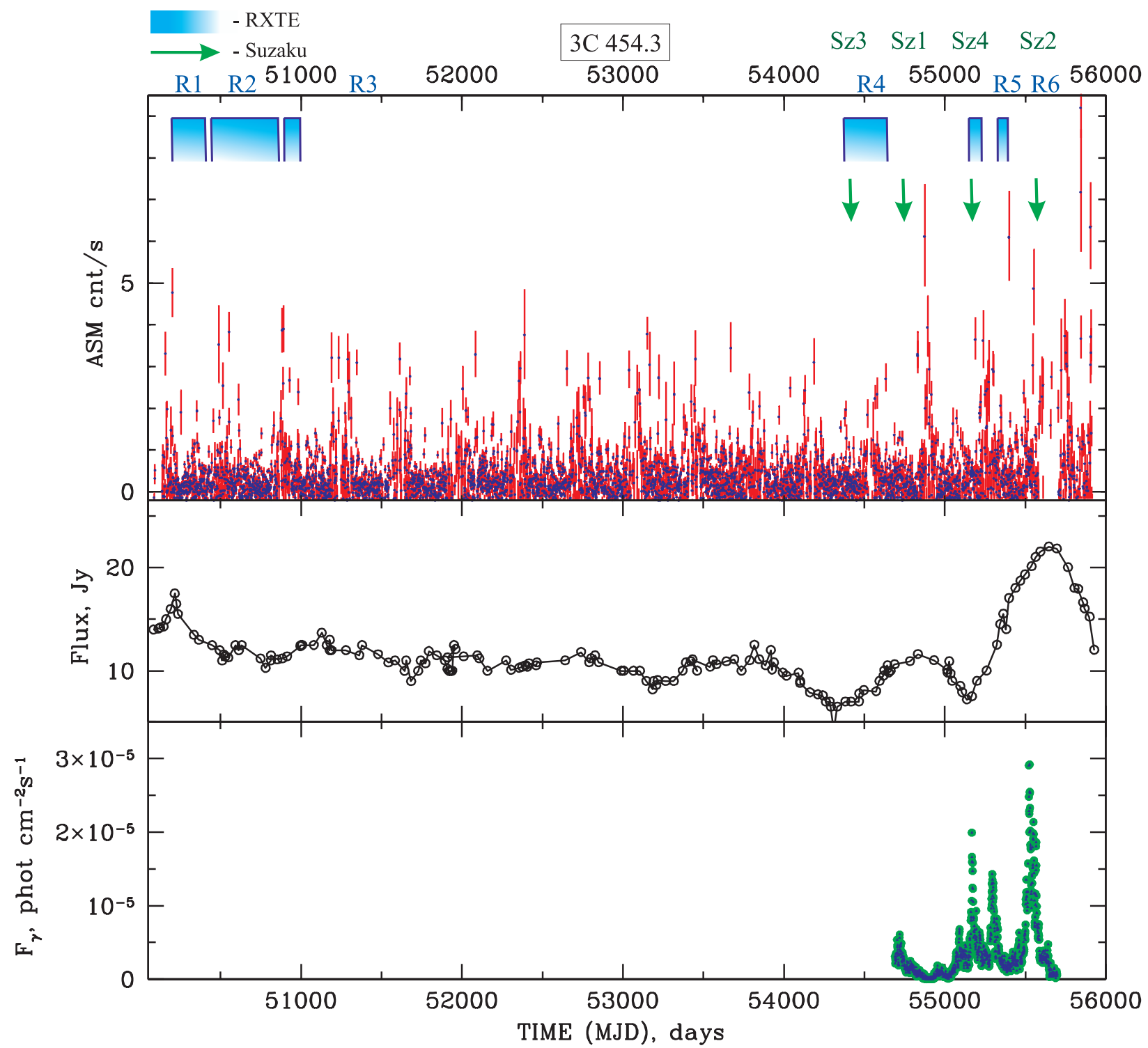

Fig. 1. Evolution of ASM/RXTE count rate $(2-12 \mathrm{keV}$, top $)$, radio $(8 \mathrm{GHz}$, middle) and $\gamma$-ray radiation $(0.1-300 \mathrm{GeV}$, bottom $)$ during $1996-2012$ observations of $3 \mathrm{C} 454.3$. Vertical arrows (at top of the panels) indicate temporal distribution of the RXTE (blue) and Suzaku (green) data sets listed in Tables 1-7.

centered on the source position of M $87\left(\alpha=12^{\mathrm{h}} 30^{\mathrm{m}} 49 \mathrm{~s} 4, \delta=\right.$ $12^{\circ} 23^{\prime} 28^{\prime \prime}$, J2000.0) (see details in Akiyama et al. 2019a). For comparison in Fig. 4 we show an image of the same area around $3 \mathrm{C} 454.3$ in the radio band $(15 \mathrm{GHz}, \mathrm{VLBA})$ at the time of our observations (see $R 2$ and Kellermann et al. 1998). It can be seen that even during a radio outburst with jet ejection, we observe a point source in X-rays. Swift data processing is described in our previous paper (Titarchuk \& Seifina 2016a).

The Chandra $(0.3-10 \mathrm{keV})$ image of the 3C 454.3 field of view is shown in Fig. 3. In the center we can see a bright pointlike X-ray source, which is well matched with the optical one $\left(\alpha=23^{\mathrm{h}} 53^{\mathrm{m}} 57.77, \delta=+16^{\circ} 08^{\prime} 52: 7, \mathrm{~J} 2000.0\right)$. This image was obtained during observations of 3C 454.3 between May 11, 2005, and June 17, 2011 (with exposure time of $277 \mathrm{ks}$ ). Figure 1 also demonstrates a very weak signature of the X-ray jet-like (elongated) structure and the minimal contamination by other point sources and diffuse emission around 3C 454.3.

On the other hand the jet-like structure in M 87 is strong and knotted. The knot, which is close to the nucleus of M 87 (so called as HST-1), is variable. Figure 5 shows a Chandra
X-ray image on April 14, 2017, with the strong HST-1 for $5 \mathrm{ks}$ exposure. Here, we indicate the nucleus, the flaring knot HST-1 (0.86" from the nucleus), knot D, and knot A.

Before proceeding to details of the spectral fitting we study the long-term behavior of 3C 454.3 and M 87, particularly in its active phases. We discuss a long-term one-day average X-ray light curve of 3C 454.3 detected by the RXTE ASM over the total lifetime of the mission (1996-2012, Fig. 1). The brightblue rectangles indicate the MJD intervals of RXTE observations used in our analysis. Vertical green arrows (top of the panel) indicate temporal distribution of the Suzaku data sets listed in Table 1. It is clear that 3C 454.3 became brighter, on average, in 2009-2010 $(R 5, R 6)$ in a soft X-ray band (1-12 keV) than in the 1996-1997 period (see $R 1, R 2$ ). Blue points show the source signal and red line indicates its error bars. The ASM monitoring observations are distributed more densely over time (1996-2012) than those of Suzaku (20072010, Abdo et al. 2010). However, it can be seen that the Suzaku observations cover the time interval with increased X-ray count-rate events. 


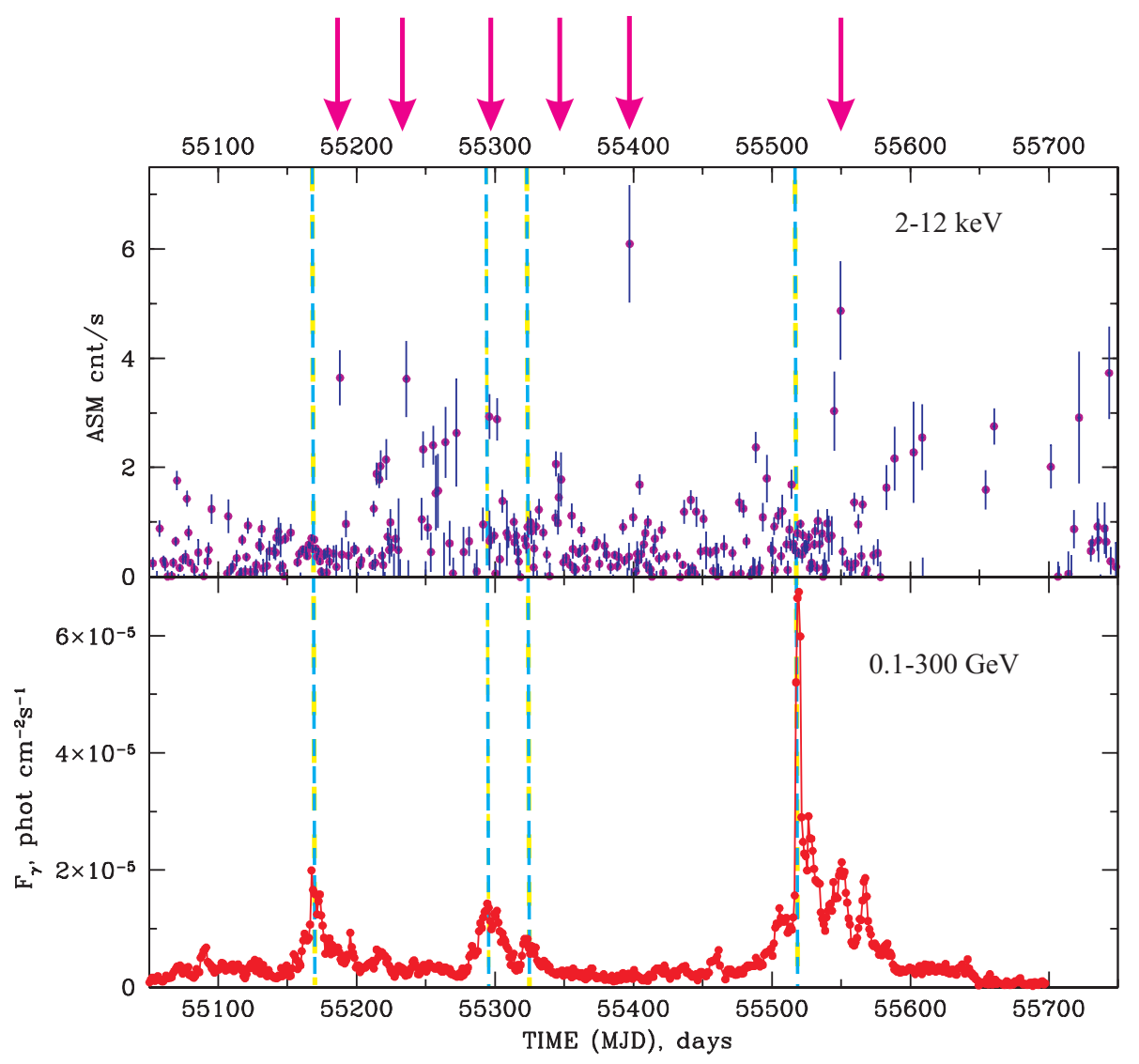

Fig. 2. Delays of peak-fluxes for outburst of 3C 454.3 observed in 2010-2012 for two bands: 2-12 keV (top) and 0.1-300 GeV (bottom). Vertical blue dashed lines mark the peak of $\gamma$-ray flux, while pink arrows (at top of the panels) indicate the peaks of X-ray bursts.
We also wish to bring attention to the synchronous light curves in radio $(8 \mathrm{GHz}$, middle panel in Fig. 1$)$ and $\gamma$-ray bands (0.1-300 GeV, bottom panel) during 1996-2012 observations of $3 \mathrm{C} 454.3$. (green) It can be seen here that although the radio flash of 3C 454.3 (middle panel) correlates with the amplification of the $\gamma$-ray radiation (bottom panel) from the source, the X-ray light curve of 3C 454.3 (top panel) shows a more frequent irregular variability, which does not correlate with global outburst in the radio and $\gamma$-ray ranges.

In Fig. 6 we display the evolution of ASM/RXTE count rate during 1996-2012 observations of M 87 (top). Vertical arrows (at the top) indicate temporal distribution of the RXTE (blue) and Suzaku (green) data sets listed in Tables 1-7. We also demonstrate the evolution of the X-ray intensity of the nucleus (green) and HST-1 (black) seen by Chandra during 2000-2008 observations of M 87 according to data taken from Harris et al. (2009) in the lower panel.

Regarding an issue of possible correlation between flares in the X-ray and $\gamma$-ray ranges (delay/lead), we did not find any correlation for the data of 3C 454.3 observed in 2010-2012 (Fig. 2) for two bands: $2-12 \mathrm{keV}$ (top) and $0.1-300 \mathrm{GeV}$ (bottom). In Fig. 2 we mark the peak of $\gamma$-ray flux by vertical blue dashed lines, while pink arrows (at top of the panels) indicate the peaks of X-ray bursts. Obviously, there is no correlation between $\gamma$ and X-ray flashes. Our conclusion is somewhat different from the conclusions of Volvach et al. (2017), but we may have used a different set of data.

\subsection{Spectral analysis}

We used different spectral models in order to test them using all available data of 3C 454.3 and M 87. We want to establish evolution between the low/hard state (LHS) and the interme- diate state (IS) using these spectral models. We investigate the Chandra, Susaku, Swift and RXTE spectra to test the following XSPEC spectral models: powerlaw, Bbody, BMC, and their possible combinations.

\subsubsection{Choice of spectral model}

As a first step, we present Suzaku and Chandra spectral data of $3 \mathrm{C} 454.3$ in the $0.3-10 \mathrm{keV}$ energy range. We establish that the thermal model (black body) fits the low-energy part well, while providing an excess emission for $E>3 \mathrm{keV}$ (e.g., for $S z 2$ and $S z 4$ spectrum, $\chi_{\text {red }}^{2}=13.2$ (1797 d.o.f.) and $\chi_{\text {red }}^{2}=13.08$ (1643), respectively, at the top part of Table 2). We find that the black body model indicates very low absorption (less than $0.9 \times 10^{20} \mathrm{~cm}^{-2}$ ) for all $S z 1-S z 4$ spectra, and moreover, that this model gives unacceptable fit quality, $\chi^{2}$, for all spectra of Swift data. However, we should note that the tbabs*power-law model provides better fits than the thermal one. No Susaku data can be fitted well by a single-component model. Indeed, a simple power-law model produces a soft excess below $0.6 \mathrm{keV}$. These significant positive residuals at low energies, less than $1.3 \mathrm{keV}$, suggest the presence of additional emission components in the spectrum. Therefore, we also tested a model consisting of blackbody and power-law components. The model parameters of this combined model are $N_{\mathrm{H}}=5 \times 10^{21} \mathrm{~cm}^{-2} ; k T_{\mathrm{bb}}=100-260 \mathrm{eV}$ and $\Gamma=1.1-2$ (see more in Table 2). The best fits of the Suzaku spectra were found using of the Bulk Motion Comptonization model (BMC XSPEC model, Titarchuk et al. 1997). We should emphasize that all Suzaku best-fit results were obtained using the same model for all spectral states. Therefore, further we applied the BMC model modified by the interstellar absorption to all our Swift and RXTE observations of 3C 454.3. 


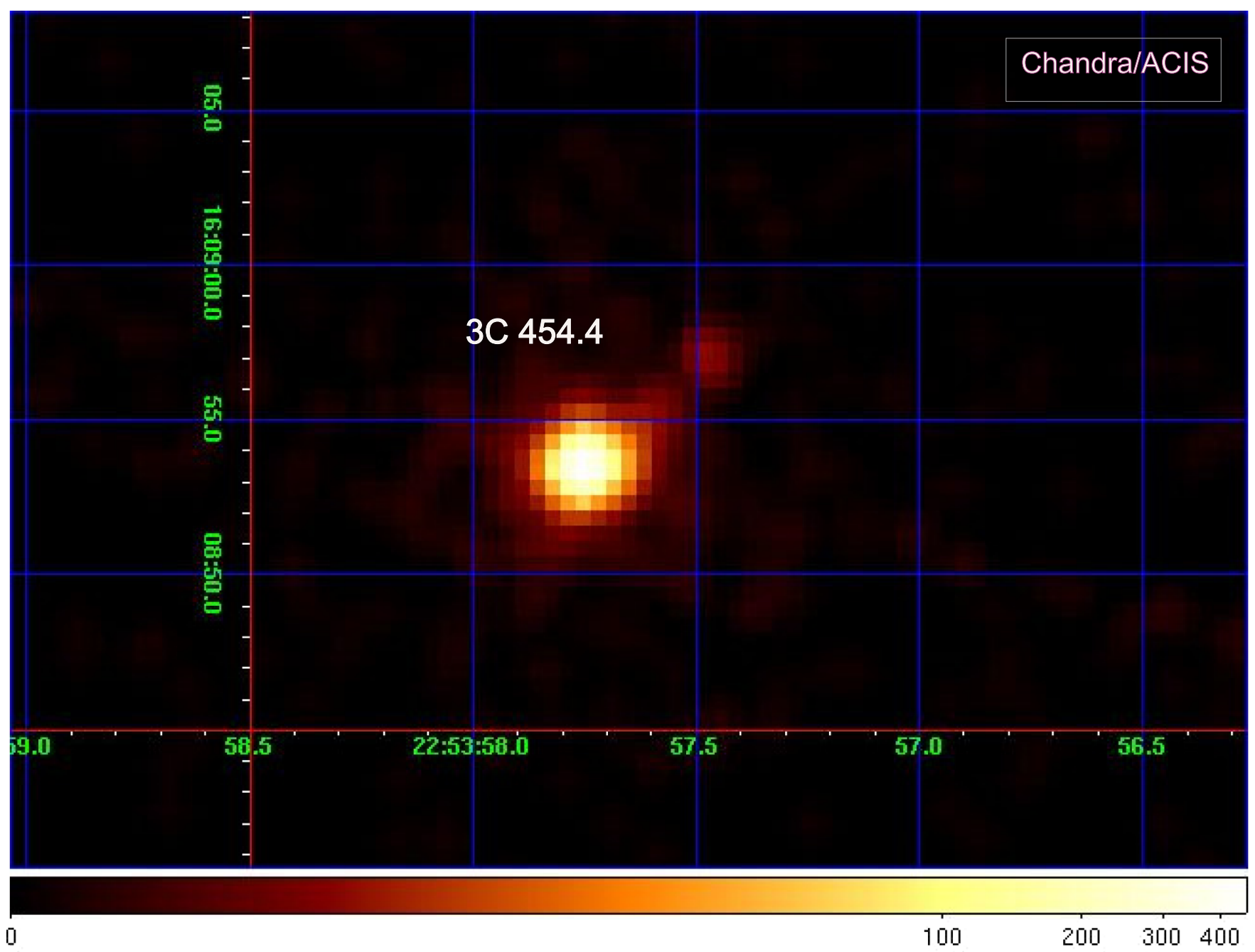

Fig. 3. Chandra/ASIS (0.3-10 keV) image of 3C 454.3 field of view on November 6, 2002 with the exposure of $4929 \mathrm{~s}(\mathrm{MJD}=52584)$.

A similar result concerning the choice of spectral model was obtained for M 87 using the Chandra spectra of the M 87 core (see Table 8). Therefore, further we applied the BMC model modified by the interstellar absorption to all our BeppoSAX, Suzaku, ASCA, Swift and RXTE observations of M 87. In Fig. 7 we show one $E F(E)$ spectral diagram for the IS using the BeppoSAX data. Interestingly, we found many emission features in the BeppoSAX spectrum and fitted the spectrum with the tbabs* (bmc $+N^{*}$ Gauss) model. However, these features may be associated with a remote shell. In fact, BeppoSAX data come from a vast area that includes both the nucleus and the jet and from the external parts of the AGN. Moreover, the spectra obtained using Chandra for a narrower region of the nucleus of M $87\left(<0.85^{\prime \prime}\right)$ no longer contain emission features (see Fig. 12).

The same conclusion was drawn for the XMM observations (Kennea et al. 2000). Therefore, we continued to work with the Chandra spectra in order to select a model for fitting the radiation of the nucleus of M87. We also proceeded with a powerlaw model; it fits the spectrum well, but the data are poorly matched by the $\chi$-square criterion $\left(\chi_{\text {red }}^{2}=0.62\right.$ for 174 d.o.f., ID $=18783$, see top of the Table 8). The thermal black body model also fits the spectrum well, however it poorly approximates the regions of $0.3-$ $1 \mathrm{keV}$ (possibly related to the underestimation of absorption) and the energy region above $7 \mathrm{keV}\left(\chi_{\text {red }}^{2}=1.5\right.$ for 176 d.o.f., ID $=21075)$. In this case, taking absorption into account unnec- essary. It is worth noting that the above models provide a soft excess below $0.3 \mathrm{keV}$. To eliminate this drawback, we used a combined model consisting of black body and power-law components. This latter model also fits the spectrum of the M 87 nucleus well, but this combined model is more phenomenological than the physical one. Therefore, in the subsequent step, we used the generic BMC model. This model describes the spectra of the M 87 nucleus well in all states (see bottom in the Table 8).

It is important to emphasize that the log-parabolic power-law models (Kardashev 1962; Massaro et al. 2004a,b, 2006) were used for analysis of the blazar spectra. Indeed, the log-parabolic model is one of the simplest ways to describe curved spectra when these show mild and nearly symmetric curvature around the maximum, instead of a sharp high-energy cut-off like that of an exponential. As it is described by Massaro et al. (2006) the log-parabolic model law has only one more parameter than a simple power-law. For example, one can see it applying Eq. (3) of Massaro et al. (2004a,b) who apply this formula for photon spectra. This appropriate formula is

$F(E)=K\left(E / E_{1}\right)^{-\left[a+b \lg \left(E / E_{1}\right)\right]}$,

in which the reference energy $E_{1}$ is fixed at $1 \mathrm{keV}$ and thus the spectrum is determined only by three parameters $K, a$ and $b$ and the energy dependent photon index is

$\Gamma(E)=a+b \lg \left(E / E_{1}\right)$. 


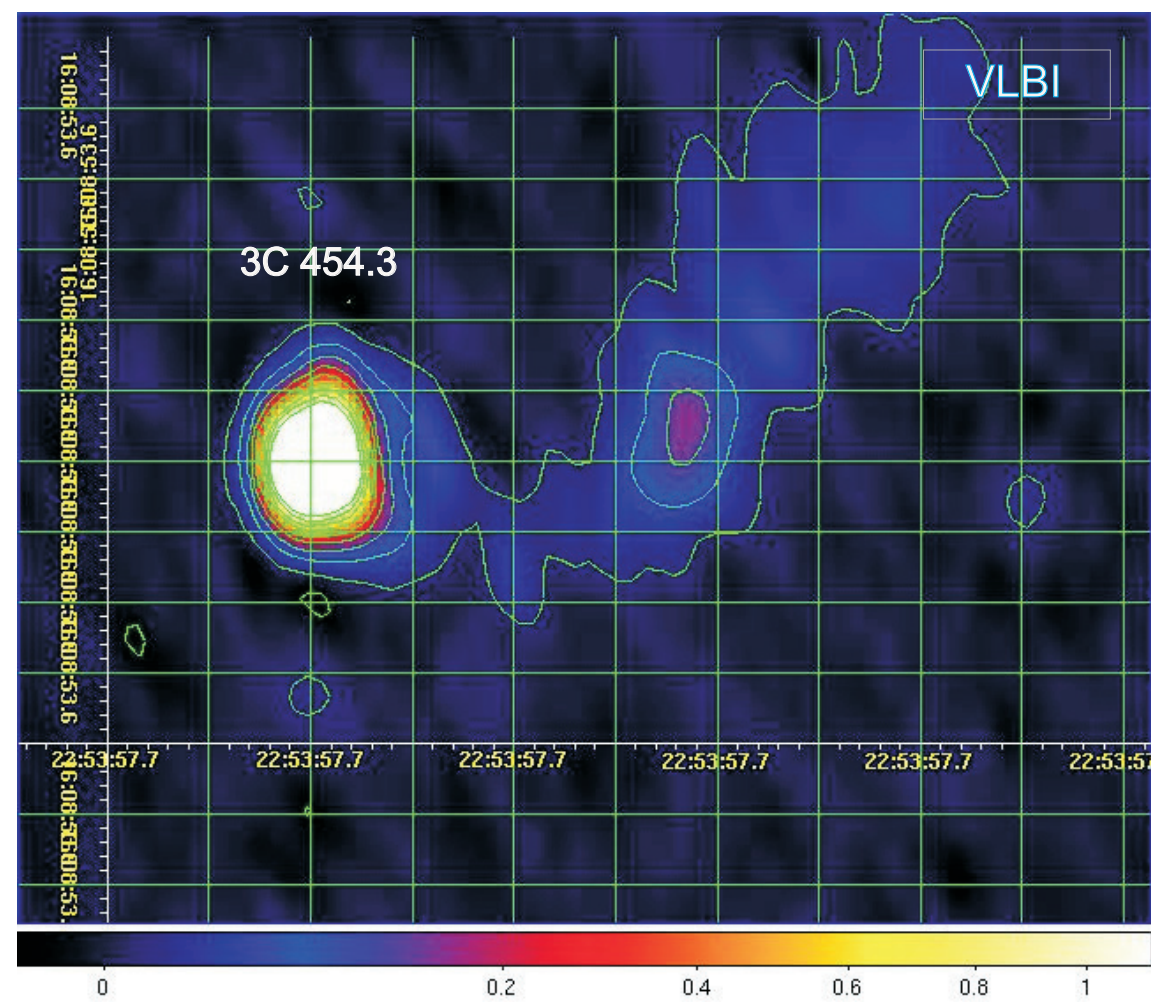

0

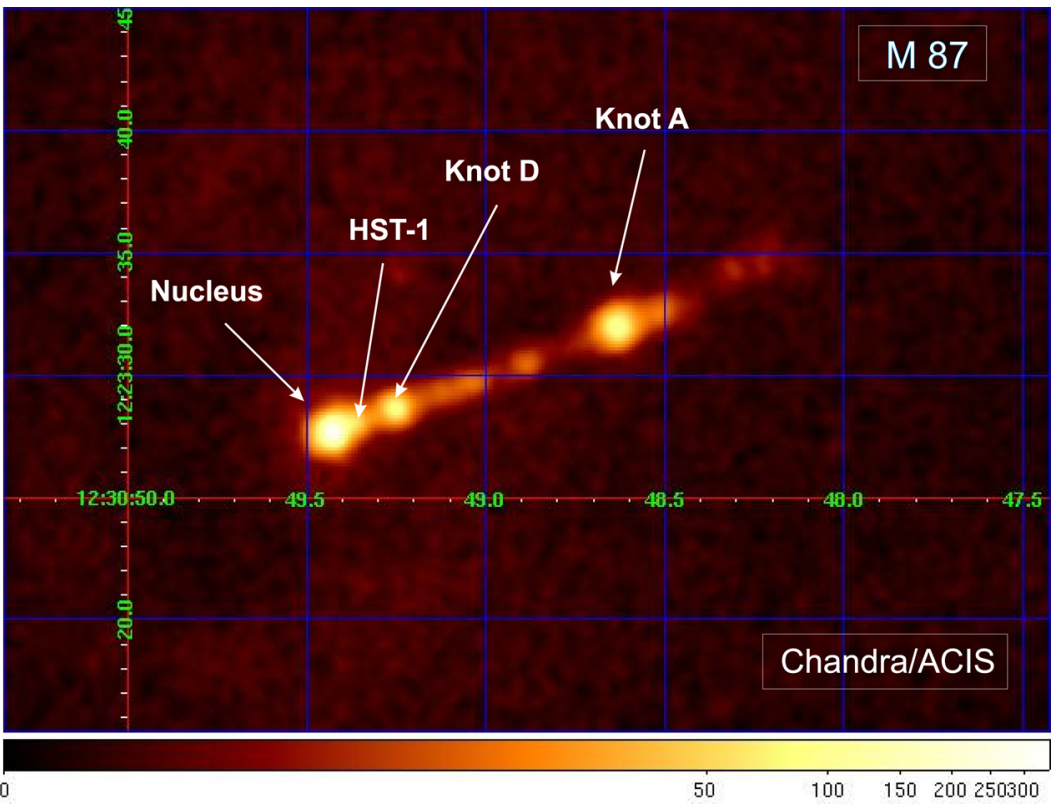

Fig. 4. Adaptively smoothed $V L B I$ image of radio emission of 3C 454.3 field detected on March 10, 1997. Contours correspond to nine logarithmic intervals in the range of $3 \times 10^{-3}-5 \%$ with respect to the brightest pixel.
Fig. 5. Chandra X-ray images on April 14, 2017, with strong HST -1 , with $5 \mathrm{ks}$ exposure. We indicate the nucleus, the flaring knot HST-1 (0.86" from the nucleus), knot D, and knot A.
However, it is easy to show that this log-parabolic model is approximated by a single power-law for large $E$ and frozen pivot $E$. In fact, if the index of this simplified broken powerlaw and log-parabolic model is too large then it leads to very high residuals at low-energies. This is a well-known effect of this simplification. The log-parabolic model often uses a $N_{\mathrm{H}}$ fixed at galactic values (Massaro et al. 2004a,b), and for sources where the internal absorption is not relevant. In fact, this is one of the motivations for not using this model in the context of the present analysis.

There are several advantages to using the BMC model with respect to other common approaches applied to studies of $\mathrm{X}$-ray spectra of accreting compact objects, including a broken power-law and the log-parabolic model. First, the BMC is by nature applicable to the general case where there is an energy gain through not only thermal Comptonization but also via dynamic (bulk) motion Comptonization (see Titarchuk et al. 1997; Laurent \& Titarchuk 1999; Shaposhnikov \& Titarchuk 2009, for details). Second, with respect to the log-parabolic model, the BMC spectral shape has an appropriate low-energy curvature, which is essential for a correct representation of the lower-energy part of the spectrum. Long-term experience with log-parabolic components shows that the model fit with this component is often inconsistent with the $N_{\mathrm{H}}$ column values and produces an unphysical component "conspiracy" with the highecut part. Specifically, when a multiplicative component highecut 


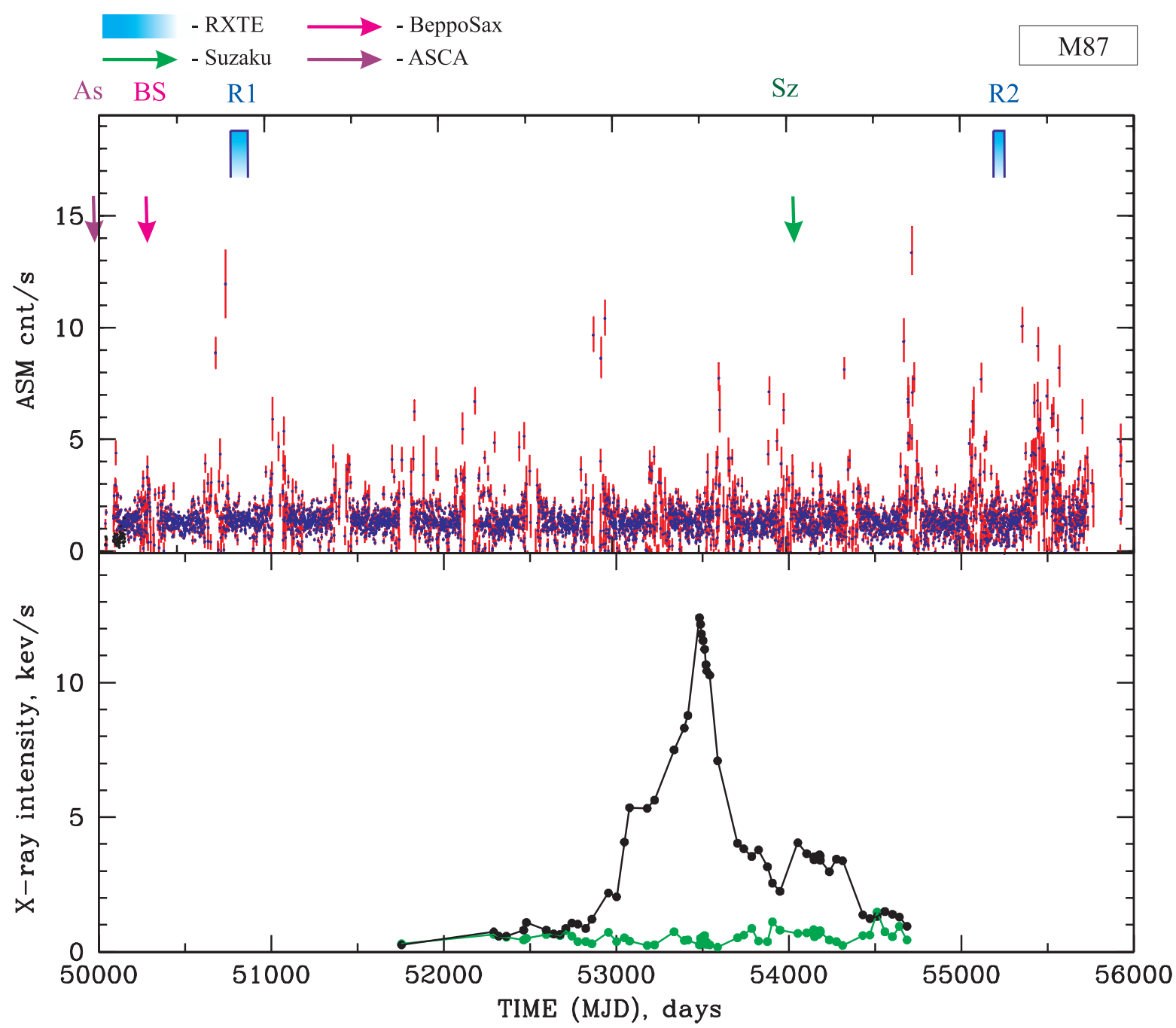

Fig. 6. Top: evolution of ASM/RXTE count rate during 1996-2012 observations of M 87. Vertical arrows (at the top of panel) indicate temporal distribution of the RXTE (blue) and Suzaku (green) data sets listed in Tables 1-7. Bottom: evolution of Chandra X-ray intensity of the nucleus (green) and HST-1 (black) during 2000-2008 observations of M 87 according to data taken from Harris et al. (2009).

is combined with the BMC, the cutoff energies $E_{\text {cut }}$ are in the expected range of $20-30 \mathrm{keV}$, while in a combination with the log-parabolic, $E_{\text {cut }}$ often goes below $10 \mathrm{keV}$, resulting in unreasonably low values of the photon index. Furthermore, implementation of the log-parabolic model makes it much harder or even impossible to correctly identify the spectral state of the source, which is an imminent task for our study. An even more important property of the BMC model is that it consistently calculates the normalization of the original "seed" component, which is expected to be an indicator of a correct mass accretion rate. We should point out that the Comptonized fraction is also properly evaluated by the BMC model.

Figure 8 shows the best-fit model of the spectrum of 3C 454.3 (top panel). The data are taken from Swift observations using the tbabs*bmc model $\left(\chi_{\text {red }}^{2}=1.02\right.$ for 770 degrees of freedom). The best-fit parameters are $\Gamma=1.63 \pm 0.02$ and $T_{\mathrm{s}}=280 \pm 20 \mathrm{eV}$. The data are denoted by black crosses, while the spectral model is presented by a red histogram. In the bottom panel we show the corresponding $\Delta \chi$ versus photon energy (in $\mathrm{keV}$ ). Using the Swift data we find that the seed temperature $k T_{\mathrm{s}}$ of the BMC model varies only slightly from 130 to $280 \mathrm{eV}$.

The spectral shape change during the spectral transition can be seen in Fig. 9, where two representative $E F(E)$ spec- tra are shown for the low/hard state (red, id =703006010) and for the intermediate one (black, id $=904003010$ ) of $3 \mathrm{C} 454.3$ detected by Suzaku. We also use the tbabs*bmc model to fit all RXTE data. In order to fit all of these spectra we use neutral column $N_{\mathrm{H}}$ fixed at $5.0 \times 10^{21} \mathrm{~cm}^{-2}$ obtained using Suzaku data (see bottom of Table 2). Figure 10 shows two $E F_{\mathrm{E}}$ spectral diagrams during the LHS (left panel) and the LHS (right panel) events in 3C 454.3; data were taken from Swift observation ID $=00031493003$ and RXTE observation ID $=20346-01-01-00$. Here, the adopted spectral model is seen to accurately describe the source spectra obtained onboard different spacecrafts for the same spectral state of 3C 454.3.

Figure 11 demonstrates two representative Chandra spectra for different states of 3C 454.3. Data are taken using observations ID $=4843$ (left panel, LHS) and ID $=3127$ (right panel, HSS) and extracted from the nuclear region (less than 125 arcsec circ) around the central source. Here, the data are shown by black crosses and the spectral model (tbabs*BMC) is displayed as a colored line. This figure shows the spectral change is typical to that observed in a BH (Galactic and extragalactic ones).

Figure 12 shows three representative spectra for different states of M 87. These Chandra energy spectra demonstrate an evolution of the source from the low/hard state to the softer states 
Table 8. Best-fit parameters of the Chandra spectra of M 87 in the $0.3-10 \mathrm{keV}$ range using the following four models: tbabs*power, tbabs*bbody, tbabs*(bbody+power) and tbabs*bmc.

\begin{tabular}{|c|c|c|c|c|}
\hline & Parameter & 11514 & 18783 & 21075 \\
\hline \multicolumn{5}{|c|}{ Model } \\
\hline tbabs & $N_{\mathrm{H}}\left(\times 10^{20} \mathrm{~cm}^{-2}\right)$ & $4.03 \pm 0.01$ & $5.4 \pm 0.6$ & $7.2 \pm 0.1$ \\
\hline \multirow[t]{3}{*}{ Power-law } & $\Gamma_{\text {pow }}$ & $1.98 \pm 0.06$ & $2.18 \pm 0.07$ & $1.96 \pm 0.07$ \\
\hline & $N_{\text {pow }}(\dagger)$ & $4.3 \pm 0.3$ & $1.9 \pm 0.2$ & $7.1 \pm 0.1$ \\
\hline & $\chi_{\text {red }}^{2}$ (d.o.f.) & $0.76(113)$ & $0.62(174)$ & 0.65 (176) \\
\hline tbabs & $N_{\mathrm{H}}\left(\times 10^{20} \mathrm{~cm}^{-2}\right)$ & $0.09 \pm 0.01$ & $0.08 \pm 0.03$ & $0.06 \pm 0.01$ \\
\hline \multirow[t]{3}{*}{ Bbody } & $T_{\mathrm{BB}}(\mathrm{keV})$ & $0.47 \pm$ & 0.50 & $0.62 \pm 0.02$ \\
\hline & $N_{\mathrm{BB}}^{(\dagger)}$ & $0.14 \pm 0.09$ & $0.07 \pm 0.01$ & $0.27 \pm 0.03$ \\
\hline & $\chi_{\text {red }}^{2}$ (d.o.f.) & $2.73(113)$ & $1.95(174)$ & 1.49 (176) \\
\hline tbabs & $N_{\mathrm{H}}\left(\times 10^{20} \mathrm{~cm}^{-2}\right)$ & $3.9 \pm 0.6$ & $3.3 \pm 0.1$ & $3.9 \pm 0.6$ \\
\hline \multirow{2}{*}{ Bbody } & $T_{\mathrm{BB}}(\mathrm{keV})$ & $0.45 \pm 0.09$ & $=0.03$ & $0.44 \pm 0.09$ \\
\hline & $N_{\mathrm{BB}}(\dagger)$ & $0.10 \pm 0.08$ & $0.02 \pm 0.01$ & $0.05 \pm 0.01$ \\
\hline \multirow[t]{3}{*}{ Power-law } & $\Gamma_{\text {pow }}$ & $1.96 \pm 0.09$ & $1.88 \pm 0.05$ & $1.83 \pm 0.09$ \\
\hline & $N_{\text {pow }}(\dagger)$ & $4.4 \pm 0.3$ & $1.26 \pm 0.6$ & $5.2 \pm 0.1$ \\
\hline & $\chi_{\text {red }}^{2}$ (d.o.f.) & $0.79(111)$ & $0.59(172)$ & $0.86(174)$ \\
\hline tbabs & $N_{\mathrm{H}}\left(\times 10^{20} \mathrm{~cm}^{-2}\right)$ & $1.0 \pm 0.1$ & $7.4 \pm 0.5$ & $1.0 \pm 0.2$ \\
\hline \multirow[t]{5}{*}{ bmc } & $\Gamma_{\mathrm{bmc}}$ & $1.94 \pm 0.07$ & $2.83 \pm 0.09$ & $2.09 \pm 0.07$ \\
\hline & $T_{\mathrm{s}}(\mathrm{e}$ & $136 \pm 7$ & $168 \pm 10$ & $220 \pm 9$ \\
\hline & & $1.08 \pm 0.02$ & $-2.29 \pm 0.07$ & $1.47 \pm 0.05$ \\
\hline & $N_{\text {bmc }}(\dagger)$ & $0.10 \pm 0.04$ & $0.50 \pm 0.04$ & $0.17 \pm 0.05$ \\
\hline & $\chi_{\text {red }}^{2}$ (d.o.f.) & $0.87(111)$ & $1.01(172)$ & $0.96(174)$ \\
\hline
\end{tabular}

Notes. Errors are given at the $90 \%$ confidence level. ${ }^{(\dagger)}$ Normalization parameters of blackbody and bmc components are in units of $L_{35}^{\text {soft }} / d_{10}^{2} \mathrm{erg} \mathrm{s}^{-1} \mathrm{kpc}^{-2}$, where $L_{35}^{\text {soft }}$ is soft photon luminosity in units of $10^{35} \mathrm{erg} \mathrm{s}^{-1}, d_{10}$ is the distance to the source in units of $10 \mathrm{kpc} . T_{\mathrm{BB}}$ and $T_{\mathrm{S}}$ are the temperatures of the blackbody and seed photon components, respectively (in keV and eV). $\Gamma_{\text {pow }}$ and $\Gamma_{\text {bmc }}$ are the indices of the power law and bmc, respectively.

which was observed in the particular dates of 2010, 2016 and 2018 (see Table 6).

We use the geometry for the X-ray spectral formation in 3C 454 and M 87 shown in Seifina et al. (2018a); see Fig. 7 there. Regarding this geometry and taking into account our X-ray spectral analysis, we see that that the soft (disk) photons illuminate the Compton cloud (CC) surrounding a $\mathrm{BH}$ hole, and matter from the $\mathrm{CC}$ is accreted onto the $\mathrm{BH}$ following a converging flow (the Bulk Comptonization region).

In Fig. 13 we present the evolution of the X-ray properties of $3 \mathrm{C} 454.3$. One can see that the photon indices change in the range from 1.3 to about 1.8 when the corresponding normalization slightly increases. These spectral evolution was observed by the RXTE during 1996-1997 outburst transition events $(R 1-R 2)$.

In addition, we present the evolution of the optical and X-ray properties of 3C 454.3 (Fig. 14): the optical light curve (in stellar magnitudes) of 3C 454.3 (top panel), the RXTE/ASM count rate, and the BMC normalization during the outburst event of 20092010 (R5-R6 sets). In the bottom panel, we see again the slight change of the photon index $\Gamma=\alpha+1$. The high X-ray flux (MJD 55550) is seen when the optical flux is low. At the same time, during the optical flash at $\mathrm{MJD}=55200$ we see correlation of the optical flux in all filters (top panel). It is important to conclude that the optical variability (e.g., MJD 55400-55550) is weakly related to X-ray variability. This could indicate that the origins of the optical and X-ray emissions are different.

Applying the Suzaku data (see black triangles in Fig. 15) we obtain that the photon index, $\Gamma$, monotonically increases from 1.5 to 2.0 when the normalization of the BMC component (or mass accretion rate) changes by a factor of 10 . We illustrate this index versus mass accretion rate behavior in Fig. 15 using RXTE, Swift, Suzaku, and Chandra observations (red squares, blue squares, black triangles, and green points, respectively).

\subsubsection{X-ray spectral modeling}

As a result of the model selection (see Sect. 3.2.1), we assume one model to fit all spectral data (Tables $1-7$ ). We briefly reiterate the physical picture described by the Comptonization model (see Titarchuk et al. 1997, ST09, and Appendix A), and its basic assumptions and parameters. The BMC Comptonization spectrum is the sum of the portion of the blackbody emission directly seen by the Earth observer [a fraction of $1 /(1+A)$ ] and a fraction of the blackbody, $f=A /(1+A)$, convolved with the up-scattering Green's function, $G\left(E, E_{0}\right)$ which is, in the BMC model, a broken power-law:

$$
F_{v}=N_{\mathrm{BMC}}\left[(1-f) * B B(E)+f * \int_{0}^{\infty} B B\left(E_{0}\right) G\left(E, E_{0}\right) \mathrm{d} E_{0}\right]
$$

It is worth emphasizing that this Green's function is characterized by the main parameter, the spectral index $\alpha=\Gamma-1$. As one can see, the BMC model has the parameters, $\alpha, A$, the seed blackbody temperature $T_{\mathrm{s}}$, and the blackbody normalization, which is proportional to the seed blackbody luminosity and inversely proportional to $D^{2}$ where $D$ is the distance to the source. We also apply a multiplicative Tbabs component characterized by an equivalent hydrogen column $N_{\mathrm{H}}$ in order to take into account absorption by neutral material. 


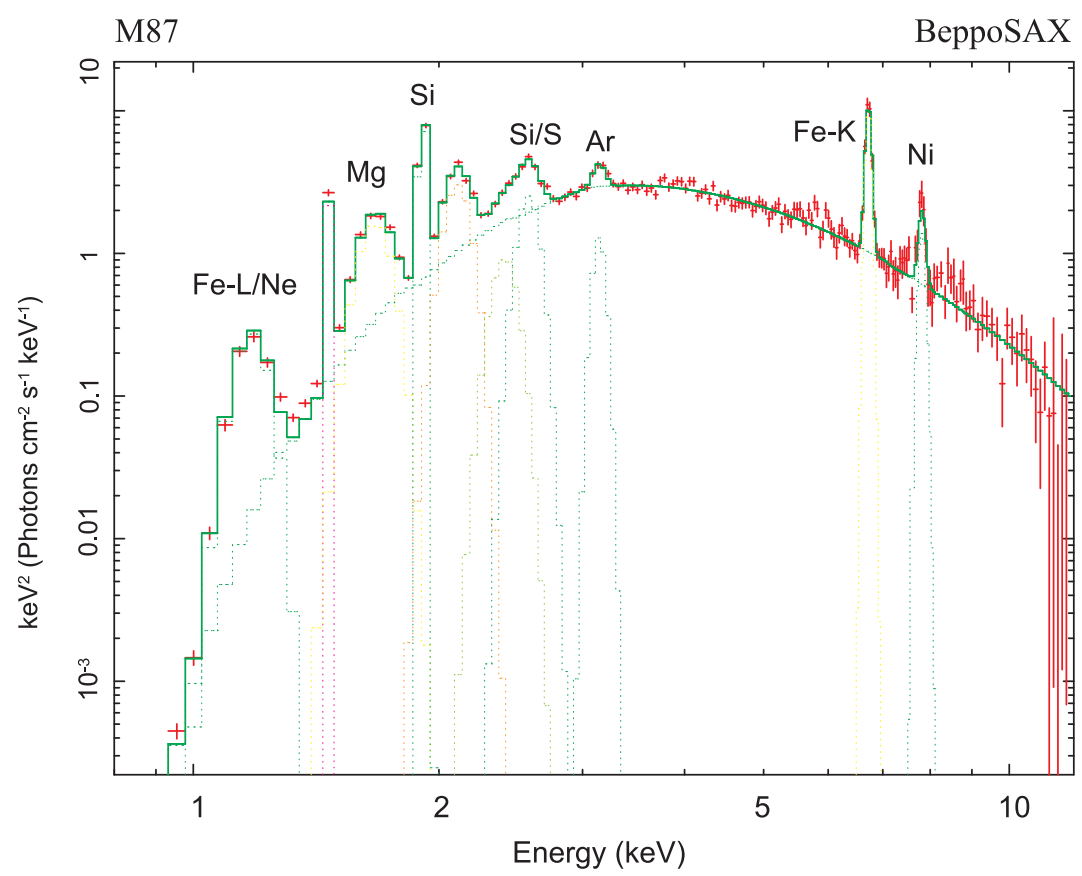

Fig. 7. Best-fit BeppoSAX spectrum of M 87 in the ISHSS state (ID $=60010001)$ using the tbabs*bmc $+N^{*}$ gauss model $\left[\chi_{\text {red }}^{2}=1.05(249\right.$ d.o.f. $\left.)\right]$. The best-fit parameters are $\Gamma=2.9 \pm 0.1$ and $T_{\mathrm{s}}=140 \pm 10 \mathrm{eV}$. (see more detalis in Table 4). The data are denoted by red crosses, while the spectral model is presented by a green histogram.

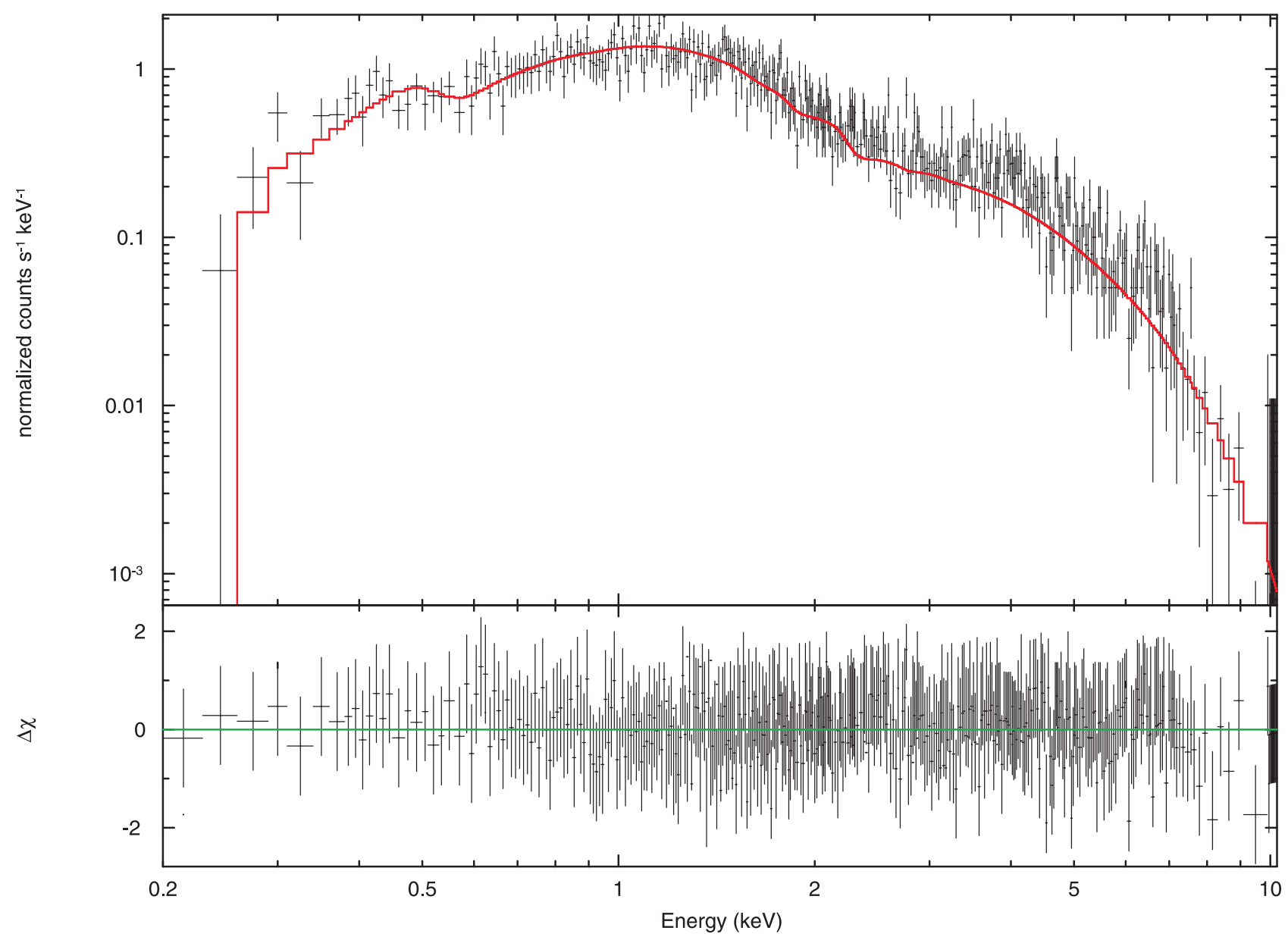

Fig. 8. Best-fit $S$ wift spectrum of $3 C 454.3$ for the IS using the tbabs*bmc model [ $\chi_{\text {red }}^{2}=1.02$ ( 770 d.o.f.)]. The best-fit parameters are $\Gamma=1.63 \pm 0.1$ and $T_{\mathrm{s}}=280 \pm 20 \mathrm{eV}$. The data are denoted by black crosses, while the spectral model is presented by a red histogram.

Figures 9 and 13 show the spectral evolution from the LHS to the IS. The BMC model successfully fits the 3C 454.3 spectra for all spectral states. The Swift spectrum obtained for 3C 454.3 in its IS using the BMC model is shown in Fig. 8. In Table 2 (at the bottom), we present the results of spectral fitting Suzaku data of 3C 454.3 using our main spectral model tbabs*bmc.

Applying the RXTE data (see Tables 7 and B.2), we find the LHS-IS transition that is related to the photon index evolution, 


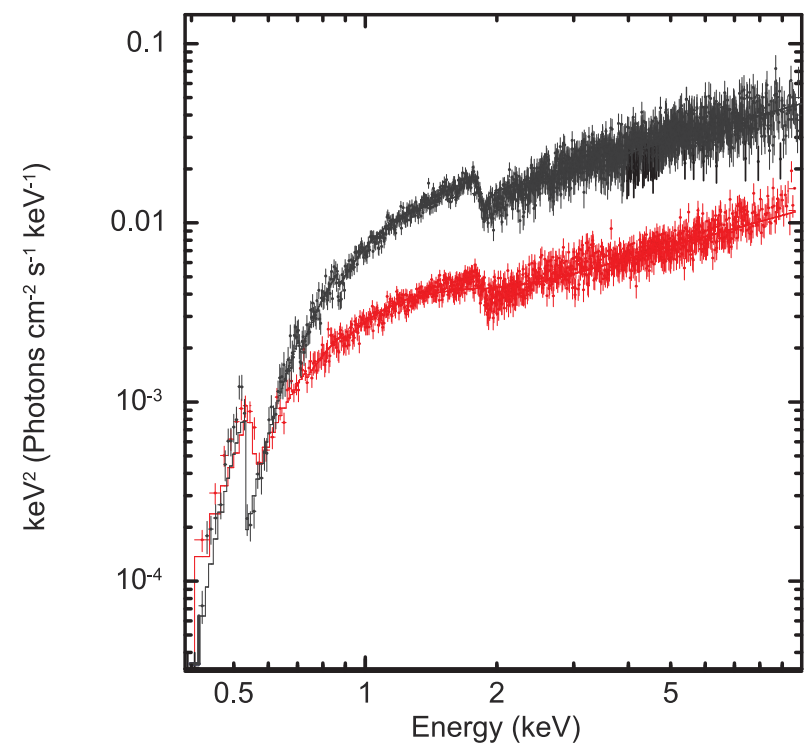

Fig. 9. Representative $E F(E)$ spectral diagrams for the low/hard state (red, id $=703006010$ ) and for the intermediate state (black, id $=904003010$ ) of 3C 454.3 obtained with Suzaku.

when $\Gamma$ changes from 1.2 to 2.1 with an increase of the seed photon normalization (proportional to the mass accretion rate). For the RXTE fits, we fix the seed photon temperature at $200 \mathrm{eV}$.

The BMC normalization, $N_{\mathrm{BMC}}$, varies by a factor of 100 , in the range of $0.1<N_{\mathrm{BMC}}<10 \times L_{35} / d_{10}^{2} \mathrm{erg} \mathrm{s}^{-1} \mathrm{kpc}^{-2}$. The Comptonized (illumination) fraction changes only slightly around $\log A \sim 1.5[f=A /(1+A)]$.

As discussed above, the spectral evolution of 3C 454.3 was previously investigated using X-ray data by many authors. In particular, Abdo et al. (2010) and Raiteri et al. (2011) studied the 2008 (Suzaku), 2008-2009 (Swift), and 2008-2010 (Swift) data sets, respectively (see also Tables 1 and 5), using single power law and absorbed power-law models, respectively. It is worth noting that Abdo et al. (2010) and Raiteri et al. (2011) fixed the hydrogen column to $N_{\mathrm{H}}=1.67 \times 10^{21} \mathrm{~cm}^{-2}$ and $N_{\mathrm{H}}=1.34 \times 10^{21} \mathrm{~cm}^{-2}$, respectively (applying the Chandra observations in 2005, see Villata et al. 2006) These qualitative models were used to establish the evolution of the spectral model parameters throughout state transitions during the outbursts.

We also found similar spectral behavior using our model and the full set of the RXTE observations. In particular, like in the aforementioned Guainazzi's and Rivers's et al. works, we also found that $3 \mathrm{C} 454.3$ demonstrates a change of the photon index $\Gamma$ between $\sim 1.2$ and 2.1 during the LHS-IS transition. Furthermore, we revealed that $\Gamma$ tends to saturate at 2.1 at high values of $N_{\text {bmc }}$. In other words we find that $\Gamma$ saturates at 2.1 when the mass accretion rate increases.

Our spectral model shows very good performance throughout all data sets. In Tables 2, B.1-B.2, and Figs. 8-11 and 13-14 we demonstrate the good performance of the BMC model in application to the Chandra, Swift, Suzaku, and RXTE data for which the reduced $\chi_{\text {red }}^{2}=\chi^{2} / N_{\text {d.o.f. }}\left(N_{\text {d.o.f. }}\right.$ is the number of degree of freedom) is less than or around 1 for all observations $\left(0.75<\chi_{\text {red }}^{2}<1.19\right)$.

We performed a similar simulation of the M 87 spectrum using the available observations. The RXTE data reveal a weak activity of the M 87 nucleus in X-rays. However, for 13 years there is a set of all states from the LHS to the HSS. In particular, M 87 spends most of its time in the HSS (1997-1998, $\Gamma \sim 3)$, although it can slowly reach LHS (2010, $\Gamma \sim 1.7-1.9)$. The data of two other satellites fill the gaps between these states. ASCA (1993) and Suzaku (2006) find the M 87 core in the IS $(\Gamma \sim 2.5)$. Finally, BeppoSAX (1996) points to the LHS $(\Gamma \sim 1.6)$.

In Table 8 we present the results of spectral fitting Chandra data of M 87 using our main spectral model tbabs*bmc. Using the RXTE data (see Table B.3) the LHS-IS transition is related when $\Gamma$ changes from 1.8 to 3 and the normalization of the seed photon increases. For the RXTE fits we fix the seed photon temperature at $200 \mathrm{eV}$. The BMC normalization, $N_{\text {BMC }}$, varies by a factor of five in the range of $0.33<N_{\text {BMC }}<$ $1.5 \times L_{37} / d_{10}^{2} \mathrm{erg} \mathrm{s}^{-1} \mathrm{kpc}^{-2}$.

As we have already discussed above, the spectral evolution of M 87 was previously investigated using X-ray data by many authors. In particular, Abdo et al. (2010) and Raiteri et al. (2011) studied data sets from 2008 (Suzaku) and 2008-2009 (Swift), and 2008-2010 (Swift), respectively (see also Tables 1 and 5), using a single power law and an absorbed power-law model, respectively. For their spectral analysis, Abdo et al. (2010) and Raiteri et al. (2011) used the hydrogen column fixed to $N_{\mathrm{H}}=1.67 \times 10^{21} \mathrm{~cm}^{-2}$ and $N_{\mathrm{H}}=1.34 \times 10^{21} \mathrm{~cm}^{-2}$, respectively, as determined by the Chandra observations in 2005 and Villata et al. (2006). These qualitative models describe the evolution of these spectral model parameters throughout the state transition during the outbursts.

We estimated a radius of the blackbody emission region, $R_{\mathrm{BB}}$, using a relation $L_{\mathrm{BB}}=4 \pi R_{\mathrm{BB}}^{2} \sigma T_{\mathrm{BB}}^{4}$, where $L_{\mathrm{BB}}$ is the seed blackbody luminosity and $\sigma$ is the Stefan-Boltzmann constant. With a distance $D$ to the source of $5 \times 10^{3} \mathrm{Mpc}$, we found $R_{\mathrm{BB}} \sim 1.6 \times 10^{12} \mathrm{~cm}$. Such a large black body region would only be expected around a supermassive black hole (SMBH) and therefore $3 \mathrm{C} 454.3$ is probably a SMBH source.

We also observed the photon index, $\Gamma$, versus the normalization of the X-ray spectrum (proportional to the mass accretion rate) in the case of $M 87$. In this case, $\Gamma$ increases from a value of 1.3 and saturates at 3 (see Fig. 16). The inclusion of high-quality observations using the Chandra data allows us to take into account the radiation of the nucleus only (without outer AGN parts and jet contaminations). In Fig. 15 we can see that Chandra observations for 3C 454.3 (green points) agree well with the general $\Gamma-$ Norm trend, while for M 87, Chandra observations (blue points) show a slightly lower normalization for the same photon indices (see Fig. 16) along with that for RXTE, Suzaku, and Swift points.

\section{Discussion}

The spectral data of 3C 454.3 and M 87 are accurately fitted by the BMC model for all analyzed LHS and IS spectra (see Figs. 8-10 and Tables 2, B.1 and B.2). Our results of spectral analysis are consistent with previous results of the spectral fitting by other authors using various X-ray observations of 3C 454.3 and $\mathrm{M} 87$.

In particular, Rivers et al. (2011) found a value for $\Gamma$ of about 1.5 on average using the RXTE data of 3C 454.3, while Giommi et al. (2006) obtained $\Gamma \sim 1.7$ using Swift observations of 3C 454.3. Chitnis et al. (2009) showed that Swift spectra of $3 \mathrm{C} 454.3$ can be fitted by an absorbed power-law model with $\Gamma$ of 1.3 using the Swift data. Rivers et al. (2013) demonstrated that soft X-ray emission can be described by an absorbed power law with $\Gamma \sim 1.6$ using the RXTE data. Raiteri et al. (2008a,b) showed that $\Gamma$ changes from 1.5 to 1.6 using the appropriate $X M M-N e w t o n$ observations. Raiteri et al. also found $\Gamma$ to vary from 1.38 to 1.85 , with an average value of $\Gamma \sim 1.6$ based on Swift data obtained in 2008-2010 (Raiteri et al. 2011). Susaku observations also demonstrated that $\Gamma=1.58 \pm 0.01$ for 2008 observations (our $S z 1$ state, 

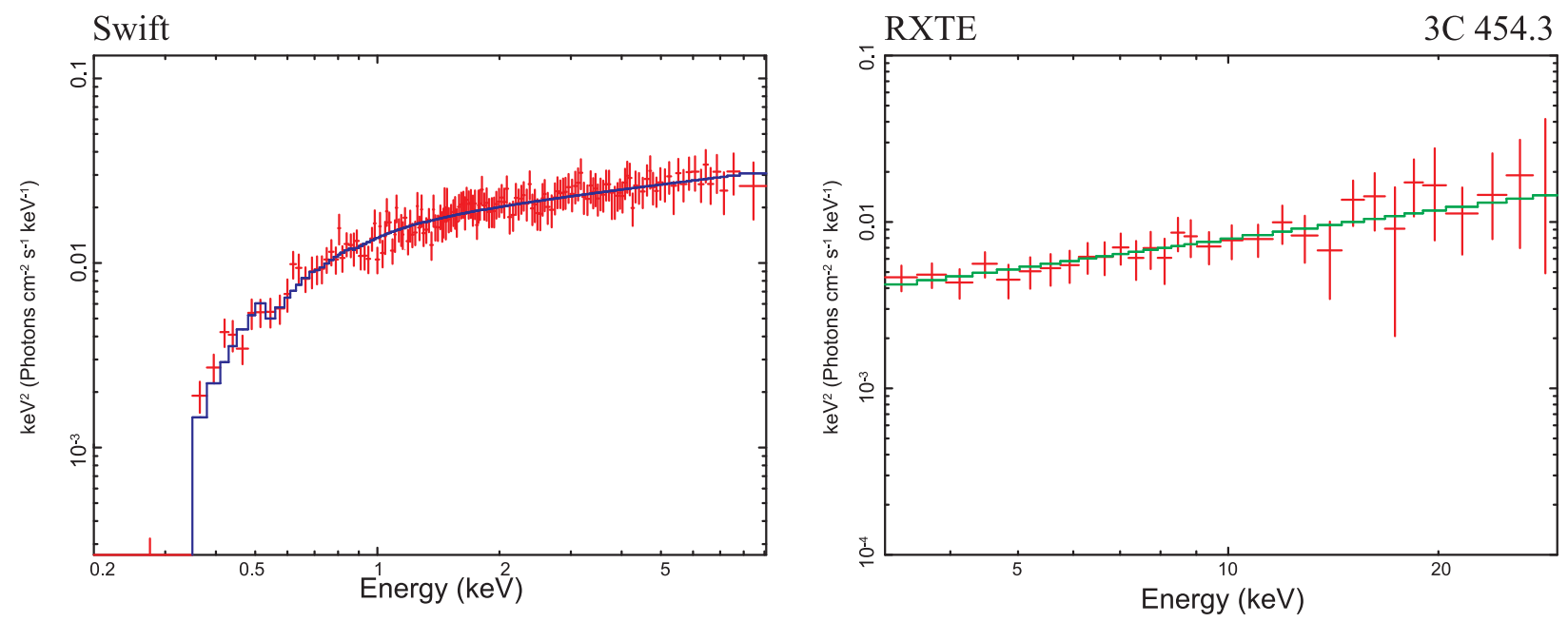

Fig. 10. Two $E F_{\mathrm{E}}$ spectral diagrams during the LHS (2009 September, 18, blue line, left panel) and the LHS (1996 November, 2 (green line, right panel)). Data taken from Swift observation 00031493003 (low/hard state) and from the RXTE observation, 20346-01-01-00 (R2 set, low/hard state).

$3 \mathrm{C} 454.3$

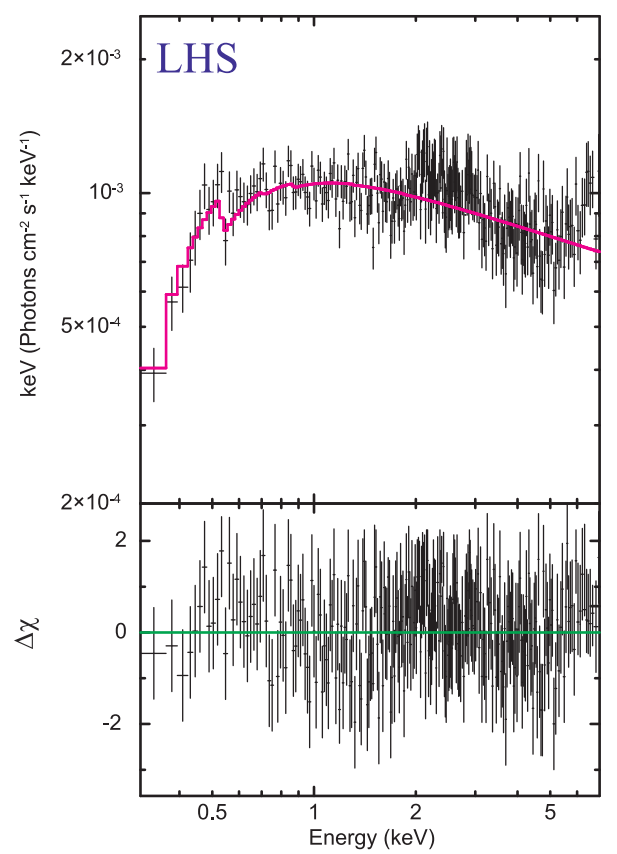

Chandra

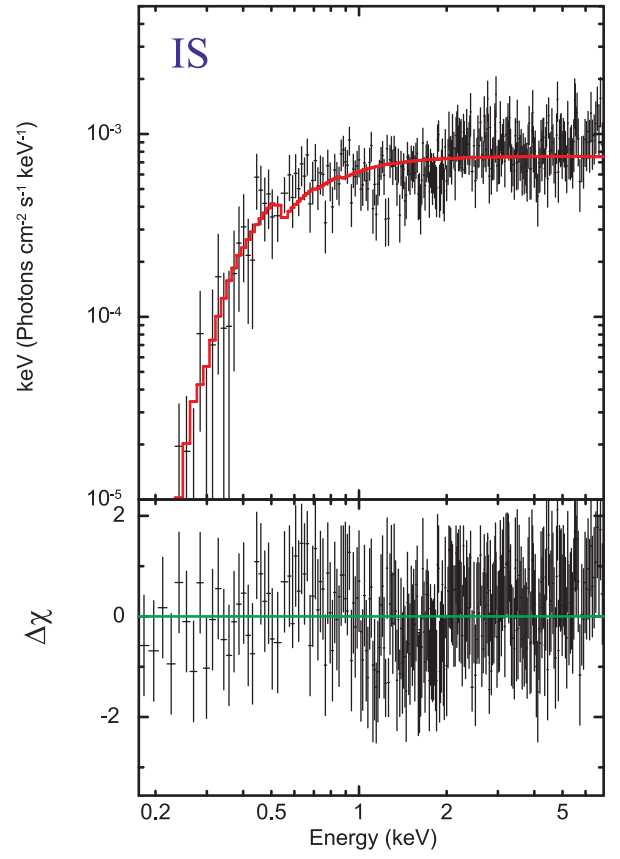

Fig. 11. Two representative $E F(E)$ spectral diagrams, for different states of 3C 454.3. Data are taken from Chandra observations ID $=4843$ (left panel, LHS) and ID $=3127$ (right panel, IS). The data are shown by black crosses and the spectral model (tbabs*BMC) is displayed as a colored line. see Table 2). The combined IBIS and Jem - X data of 3C 454.3 obtained onboard INTEGRAL gave $\Gamma=1.8$ (Pian et al. 2006). Finally, Pacciani et al. (2010) found that $\Gamma$ was very close to 1.7 using the RXTE data and $\Gamma=1.5-1.7$ using Swift/XRT observations of 3C 454.3.

For M 87, Wilson \& Yang (2002) described the spectrum of the nucleus obtained using Chandra by a simple power-law model and found that the values of the photon index vary from 2 to 3 , which is in good agreement with our results.

\subsection{Timing analysis}

We examined archival RXTE/ASM observations of M87 obtained from January 6, 1996, to December 28, 2011. The ASM light curve is presented in the top panel of Fig. 6. This light curve was analyzed using the powspec task from FTOOLS 6.26.1. We generated power density spectra (PDSs) in the $10^{-7}-10^{-3} \mathrm{~Hz}$ frequency range and subtracted the contribution due to Poissonian statistics for all PDSs. To model PDSs we used the QDP/PLT plotting package. The PDS continuum shape is usually characterized by band-limited noise shape, which is well presented by an empirical model $P_{\mathrm{X}} \sim\left(1.0+(x)^{2}\right)^{- \text {in }}$ (KING model in QDP/PLT plotter). The parameter in is a slope of PDS continuum. As a result, we find a statistically significant $(3 \sigma)$ feature at the frequency $v_{\max } \sim 5 \times 10^{-7} \mathrm{~Hz}$ (see Fig. 17) with a time bin of $\sim 100 \mathrm{ks}$. One can estimate an upper limit of a $\mathrm{BH}$ mass magnitude using this PDS feature as follows

$v_{\max } \sim V_{\mathrm{pl}} / L_{\mathrm{tr}}=10^{8} \frac{\left[V_{\mathrm{pl}} /\left(10^{8} \mathrm{~cm} \mathrm{~s}^{-1}\right)\right]}{L(\mathrm{~cm})} \mathrm{cm} \mathrm{s}^{-1}$,

where $L_{\mathrm{tr}}$ is the size of the Compton cloud (CC) in centimeters (or the transition layer) and $V_{\mathrm{pl}}$ is a plasma velocity in the $\mathrm{CC}$ of 
M87

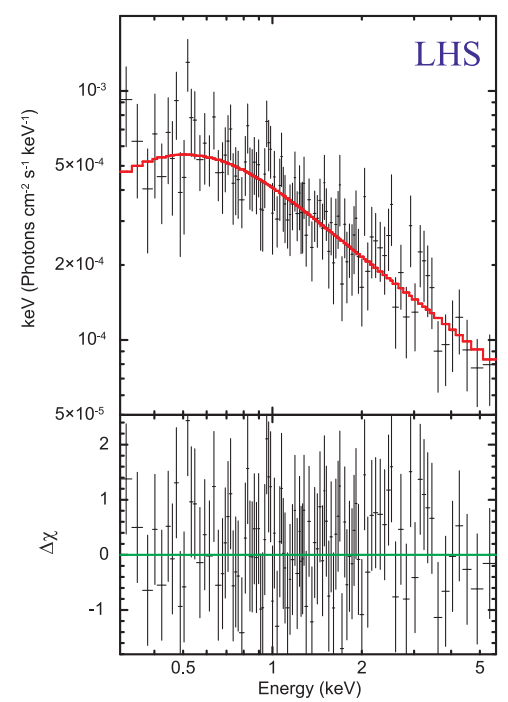

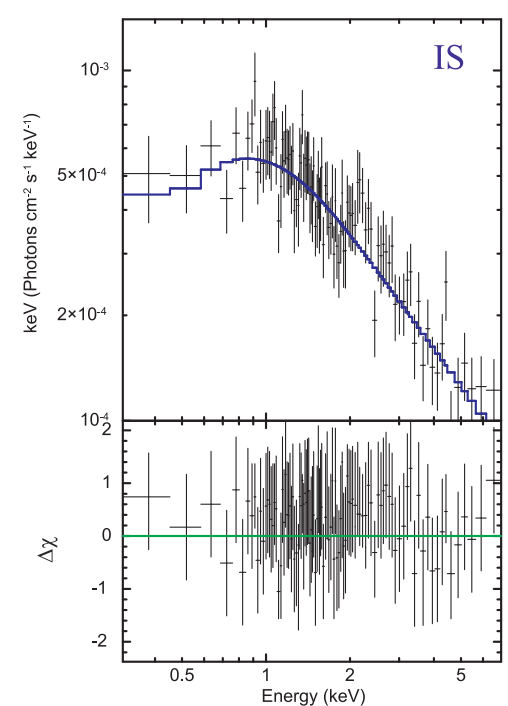

Chandra

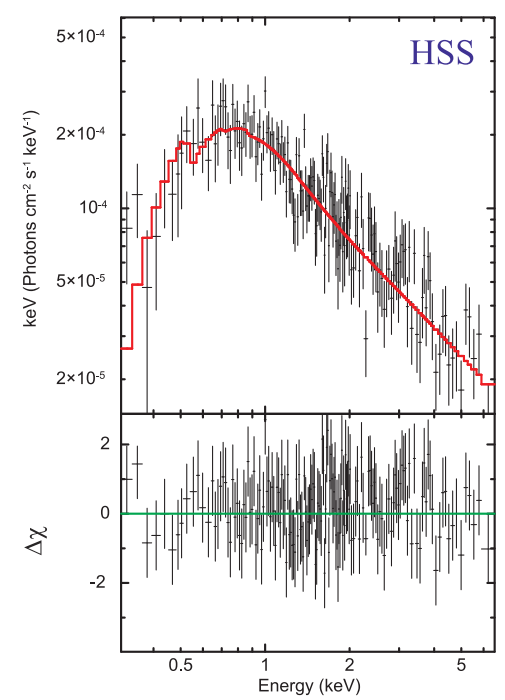

Fig. 12. Three representative energy spectra for different states of M 87. Data were taken from Chandra observations ID $=11514$ (left panel, LHS), $\mathrm{ID}=21075$ (central panel, IS), and ID = 18783 (right panel, HSS). The data are shown by black crosses and the spectral model (tbabs*BMC) is displayed as a colored line.

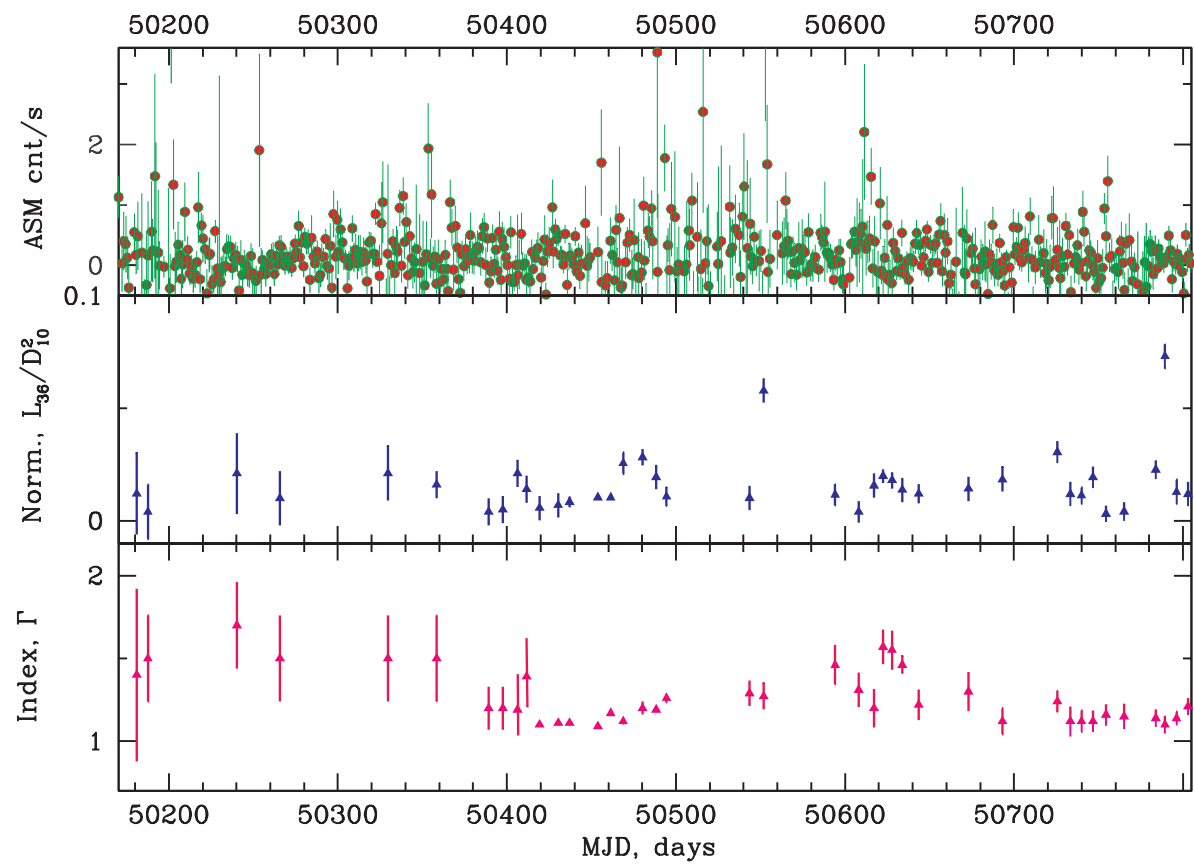

Fig. 13. Top: evolution of the RXTE/ASM count rate for 3C 454. Middle: BMC normalization for 3C 454 during 1996-1997 outburst events $(R 1-R 2$ sets). Bottom: evolution of the photon index $\Gamma=\alpha+1$. the order of $10^{8} \mathrm{~cm} \mathrm{~s}^{-1}$ related to a typical plasma temperature of the order of $10 \mathrm{keV}$ (see Shaposhnikov \& Titarchuk 2009). We use a value of the maximum of $v *$ PDS frequency which is $\sim 5 \times$ $10^{-7} \mathrm{~Hz}$ in order to estimate a $\mathrm{BH}$ mass in $\mathrm{M} 87$ :

$L_{\mathrm{tr}} \sim \frac{V_{\mathrm{pl}}}{v_{\max }} \sim 2 \times 10^{14} \frac{V_{\mathrm{pl}} / 10^{8} \mathrm{~cm} \mathrm{~s}^{-1}}{v_{\max } / 5 \times 10^{-7} \mathrm{~Hz}} \mathrm{~cm}$

One can easily compare this size $L_{\mathrm{tr}}$ with the Schwarzschild radius $R_{\mathrm{S}}$ and find that

$M_{87}<L / R_{\mathrm{S}, \odot} \sim 0.6 \times 10^{9}$ solar masses.

This BH estimate in M 87 is at least one order of a magnitude lower than that obtained by other methods for M 87 (see Akiyama et al. 2019b). In Sect. 4.3 we show that $m_{87}=M_{87} / M_{\odot}$ can be more precisely obtained using the scaling method.

\subsection{Saturation of the index as a signature of a $B H$}

We applied our analysis in order to find the evolution of $\Gamma$ in 3C 454.3 and M 87, and found that $\Gamma$ saturates with the mass accretion rate, $\dot{M}$. ST09 demonstrates this index saturation is an indication of the converging flow $(\mathrm{CF})$ into a $\mathrm{BH}$. In fact, the spectral index $\alpha \sim Y^{-1}$ is inverselly proportional to the Comptonization parameter, $Y$ which is a product of an average number of scatterings $N$ in the $\mathrm{CF}$ and an efficiency of the energy gain at any scattering in average $\eta$ (see Laurent \& Titarchuk 2007). But for the converging flow the efficient number of scattering, $N$ is proportional to the dimensionless mass accretion rate $\dot{m}$ (or the optical depth $\tau$, not like $\tau^{2}$ in the thermal plasma, see e.g. Rybick \& Lightman 1979) and the energy gain $\eta \propto \dot{m}^{-1}$. Thus one obtains that $\alpha$ saturates 


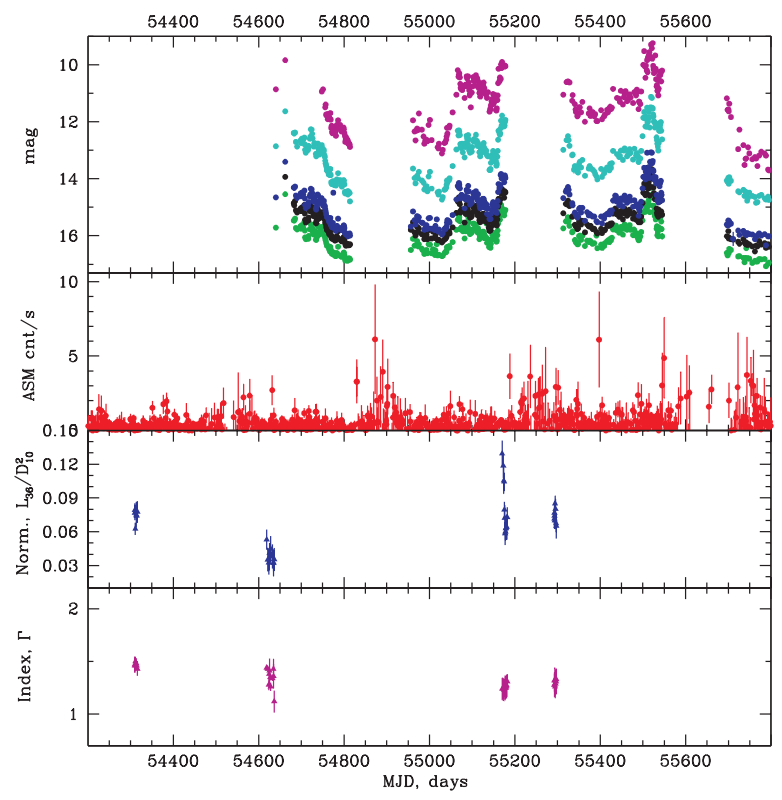

Fig. 14. Top: evolution of the optical flux (in stellar magnitudes) of 3C 454.3 in $B$-filter (green), $V$-filter (black), $R$-filter (blue), $J$-filter (bright blue), and $K$-filter (pink). Second row: RXTE/ASM count rate. Third row: BMC normalization during 2009-2010 outburst events (R5-R6 sets). Bottom: evolution of the photon index $\Gamma=\alpha+1$.

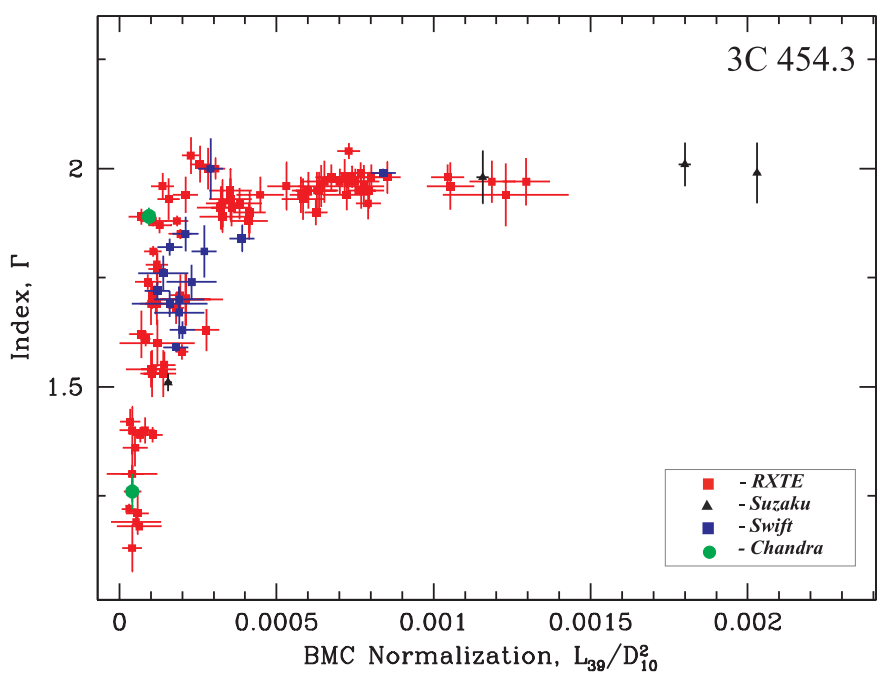

Fig. 15. 3C 454: correlations of photon index $\Gamma(=\alpha+1)$ vs. BMC normalization, $N_{\text {BMC }}$ (proportional to mass accretion rate) in units of $L_{39} / D_{10}^{2}$. Red and blue squares show RXTE and Swift observations, respectively, while blue triangles and green points correspond to Suzaku and Chandra observations, respectively.

when $\dot{m}$ increases. This is precisely what we see in Figs. 18 and 19. Another words, when the normalization parameter, proportional the mass accretion rate, $\dot{m}$ increases the photon index $\Gamma=\alpha+1$ saturates. Titarchuk \& Zannias (1998), hereafter TZ98, semi-analytically discovered this saturation effect. Later Laurent \& Titarchuk (1999, 2011; hereafter LT99 and LT11, respectively) confirmed it using Monte Carlo simulations.

It should be also noted that Titarchuk et al. (1998), hereafter TLM98, demonstrated that the innermost part of the accretion flow (the so-called transition layer) shrank when $\dot{M}$ increased.

Observations of many Galactic BHs (GBHs) and their X-ray spectral analysis (see ST09, Titarchuk \& Seifina 2009; Seifina \& Titarchuk 2010; Seifina et al. 2014; hereafter STS14)

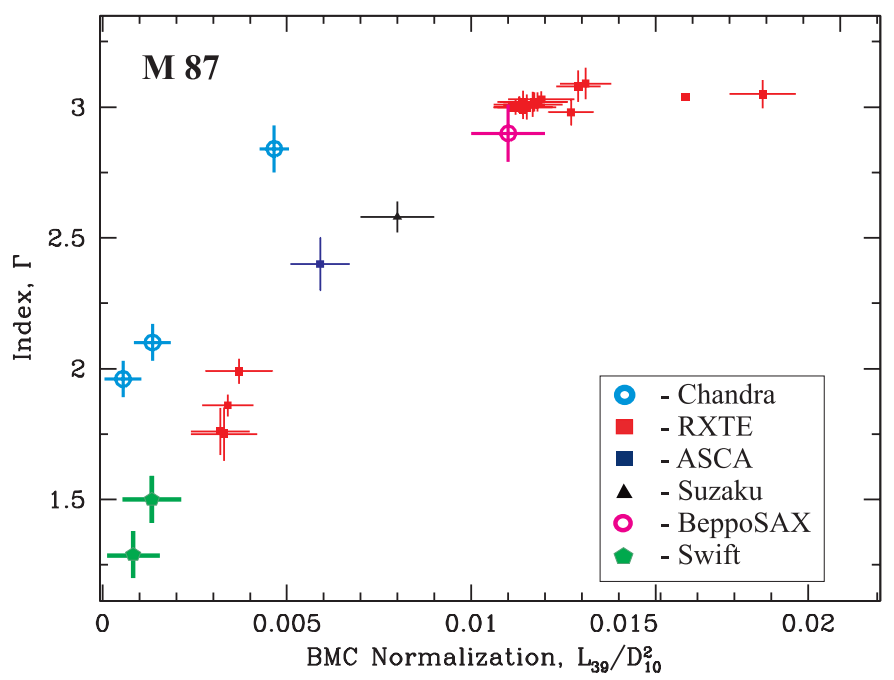

Fig. 16. $\mathrm{M} 87$ : correlations of photon index $\Gamma(=\alpha+1)$ vs. BMC normalization, $N_{\mathrm{BMC}}$ (proportional to mass accretion rate) in units of $L_{39} / D_{10}^{2}$. Red and blue squares show RXTE and ASCA observations, respectively, green points correspond to the Swift observations, black triangles correspond to Suzaku data, a pink circle denotes a point related to BeppoSAX observations, and blue circles show those related to Chandra observations.

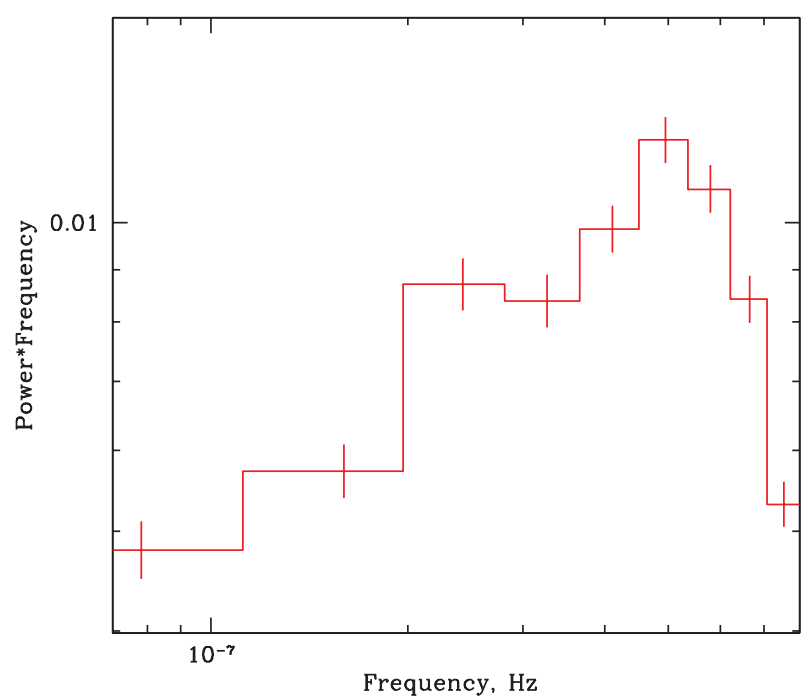

Fig. 17. Representative power spectrum of M 87. Data are taken from RXTE/ASM observations (1996-2011) with a time bin of $100 \mathrm{ks}$.

confirm this TZ98 prediction. For 3C 454.3, we also reveal that $\Gamma$ monotonically increases from 1.2 and then finally saturates at a value of 2.1 (see Fig. 15 for 3C 454.3). For M 87, the photon index increases from 1.3 and then saturates at 3 . The index- $\dot{M}$ correlations found in 3C 454.3 and M 87 allow us to estimate $\mathrm{BH}$ masses in these sources by scaling these correlations with those detected in a number of GBHs and extragalactic sources (see details below in Sect. 4.3 and in Appendix B).

\subsection{An estimate of $B H$ mass in $3 C 454.3$ and $M 87$}

A previous mass estimate of a central engine in 3C 454.3 was made using a spectroscopic method applied to multiwavelength data (see Gupta et al. 2017; Woo \& Urry 2002; Liu et al. 2006; Sbarrato et al. 2012; Gu et al. 2001). Particularly, Gupta et al. estimated a BH mass of 3C 454.3 (see also Vestergaard \& Osmer 2009) using optical emission (the broad 

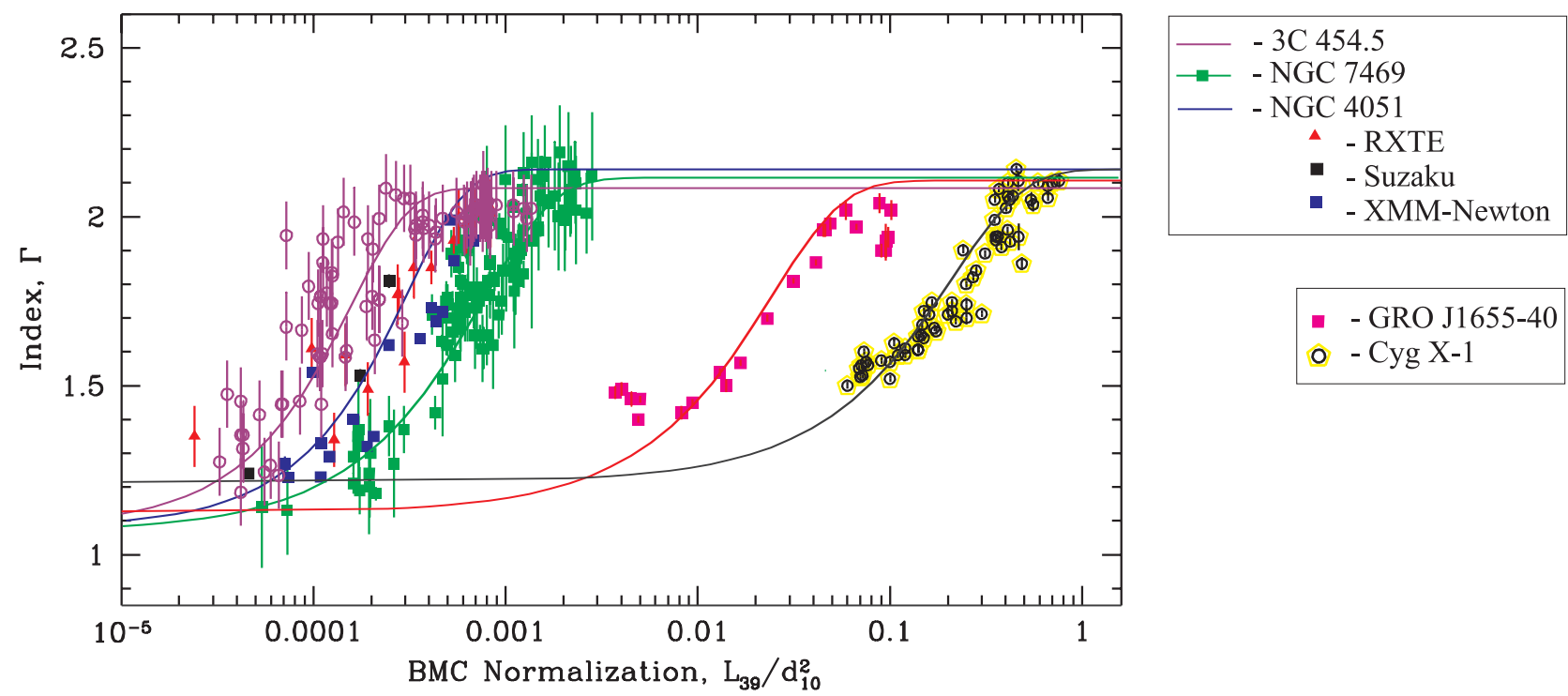

Fig. 18. Scaling of the photon index $\Gamma$ vs. the normalization $N_{\mathrm{BMC}}$ for $3 \mathrm{C} 454.3$ (violet line - target source) as well as NGC 4051 , NGC 7469, GRO J1655-40, and Cygnus X-1 (reference sources). Red triangles stand for RXTE, black squares - Suzaku and blue squares for XMM-Newton data for NGC 4051, while green squares mark RXTE data for NGC 7469. Pink squares and yellow-black circles correspond to RXTE data for GRO J1655-40 and Cygnus X-1, respectively.

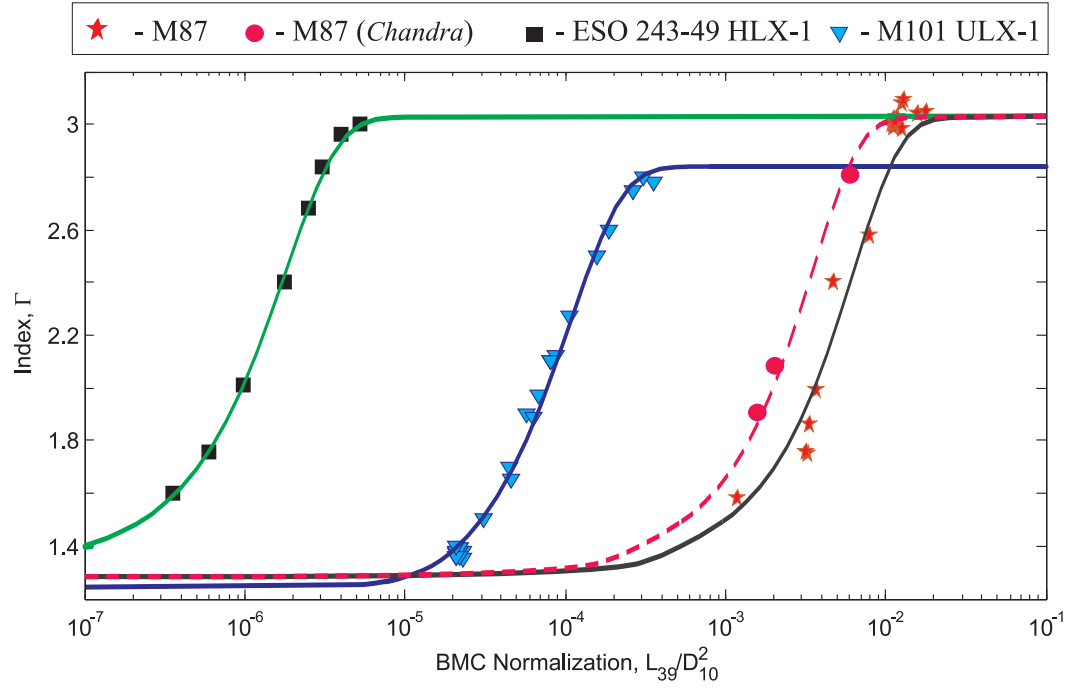

Fig. 19. Scaling of the photon index $\Gamma$ vs. the normalization $N_{\mathrm{BMC}}$ for M 87 (black line - target source) using Galactic sources: XTEJ1550-564, 4U 1630-472 and H 1743-322 (reference sources). Pink crosses stand for RXTE data of 4U 1630-472, green circles mark H 1743-322 data, and blue squares indicate XTE J1550564 data. Pink dashed line indicates the $\Gamma$-Norm correlation corrected for a nucleus emission from M 87 using Chandra data (pink points). For the scaling of a BH mass of M 87 we used this corrected $\Gamma$-Norm track (see details in the text).
Mg II line width and the continuum luminosity at $3000 \AA$ ). We estimated a $\mathrm{BH}$ mass, $M_{\mathrm{BH}}$, in 3C 454.3 applying X-ray data. In order to estimate $M_{\mathrm{BH}}$ we chose two Galactic sources (GRO J1655-40, Cygnus X-1 (see ST09)) and an extragalactic source (NGC 4051; Seifina et al. 2014, hereafter SCT18) as the reference sources, whose $\mathrm{BH}$ masses and distances are well established now.

A BH mass for GRO J1655-40 was estimated using dynamical methods. For a BH mass estimate of 3C 454.3 we also used the BMC normalizations, $N_{\mathrm{BMC}}$ of these reference sources. As a result, we scale the index versus $N_{\mathrm{BMC}}$ correlations for the target and reference sources, NGC 4051 and NGC 7469 (see Fig. 18). The value of the index saturation is almost the same, $\Gamma \sim 2$ for all these target and reference sources. We apply the correlations found in these four reference sources to make a comprehensive cross-check of a BH mass estimate for 3C 454.3.

For M87, a $\mathrm{BH}$ mass estimate turns out to be highly dependent on the accuracy of the distance to it. The distance to M87 has been estimated using several independent techniques which include measurement of the luminosity of plan- etary nebulae, comparison with nearby galaxies for which a distance is estimated using standard candles such as cepheid variables, and the linear size distribution of globular clusters (SBF method). This yields a distance of $16.6 \pm 2.3 \mathrm{Mpc}$ (Blakeslee et al. 2009). Furthermore, the tip of the red-giant branch method (Bird et al. 2010) using individually resolved red giant stars (TRGB method) yields a distance of $16.7 \pm 0.9 \mathrm{Mpc}$ (Bird et al. 2010). It should be noted that the SBF measurements by Blakeslee et al. (2009) used the HST ACS-VCS data, while the TRGB measurements were based on data from the Next Generation Virgo Cluster Survey (NGVCS) obtained using groundbased adaptive-optics with the Canada French Hawaii Telescope (Cantiello et al. 2018).

These SBF and TRGB measurements are consistent with each other, and their weighted averages yield a distance of $16.4 \pm 0.5 \mathrm{Mpc}$ (Bird et al. 2010). Using this distance and the modeling of surface brightness and stellar velocity dispersion at optical wavelengths (Gebhardt \& Thomas 2009; Gebhardt et al. 2011), a BH mass for M 87 of $6.2_{-0.6}^{+1.1} \times 10^{9} M_{\odot}$ was inferred (Akiyama et al. 2019b). On the other hand, mass measurements 


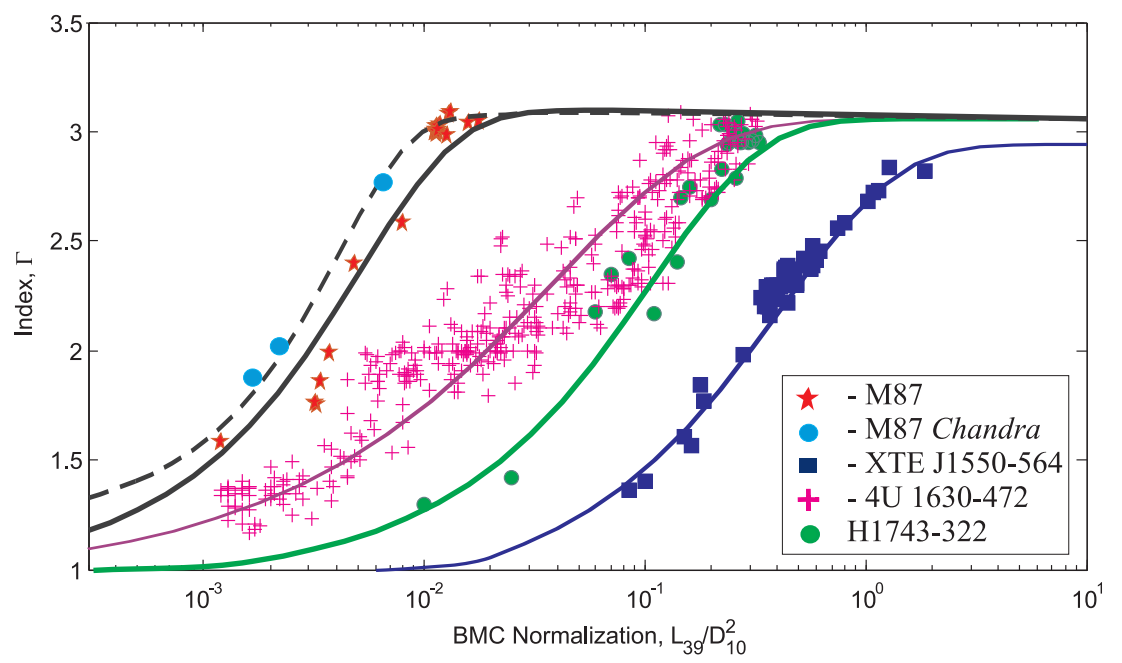

Fig. 20. Scaling of the photon index $\Gamma$ vs. the normalization $N_{\text {BMC }}$ for M87 (black line - target source) using extragalactic sources: ESO 243-49 HLX-1 and M 101 ULX-1 (reference sources). Black squares stand for RXTE data of ESO 243-49 HLX-1 and blue triangles indicate M 101 ULX-1 data. The black dashed line indicates the $\Gamma$-Norm correlation corrected for a nucleus emission from M87 using Chandra data (bright blue points). For the scaling of $\mathrm{BH}$ mass of M87 we used this corrected $\Gamma$-normalization track.

Table 9. Parameterizations for reference and target sources.

\begin{tabular}{lccccc}
\hline \hline Reference source & $\mathcal{A}$ & $\mathcal{B}$ & $\mathcal{D}$ & $x_{\text {tr }}$ & $\beta$ \\
\hline GRO J1655-40 & $2.03 \pm 0.02$ & $0.45 \pm 0.03$ & 1.0 & $0.07 \pm 0.02$ & $1.9 \pm 0.2$ \\
Cyg X-1 & $2.09 \pm 0.01$ & $0.52 \pm 0.02$ & 1.0 & $0.4 \pm 0.1$ & $3.5 \pm 0.1$ \\
NGC 4051 & $2.05 \pm 0.07$ & $0.61 \pm 0.08$ & 1.0 & {$[9.54 \pm 0.2] \times 10^{-4}$} & $0.52 \pm 0.09$ \\
NGC 7469 & $2.04 \pm 0.06$ & $0.62 \pm 0.03$ & 1.0 & $1.25 \pm 0.04$ & $0.62 \pm 0.04$ \\
XTE J1550-564 RISE 1998 & $2.84 \pm 0.08$ & $1.8 \pm 0.3$ & 1.0 & $0.132 \pm 0.004$ & $0.61 \pm 0.02$ \\
H 1743-322 RISE 2003 & $2.97 \pm 0.07$ & $1.27 \pm 0.08$ & 1.0 & $0.053 \pm 0.001$ & $0.62 \pm 0.04$ \\
4U 1630-472 & $2.88 \pm 0.06$ & $1.29 \pm 0.07$ & 1.0 & $0.045 \pm 0.002$ & $0.64 \pm 0.03$ \\
M 101 ULX-1 & $2.88 \pm 0.06$ & $1.29 \pm 0.07$ & 1.0 & {$[4.2 \pm 0.2] \times 10^{-4}$} & $0.61 \pm 0.03$ \\
ESO 243-49 HLX-1 & $3.00 \pm 0.04$ & $1.27 \pm 0.05$ & 1.0 & $4.25 \pm 0.03$ & $0.62 \pm 0.05$ \\
\hline Target source & & & & & \\
\hline 3C 454.3 & $2.01 \pm 0.06$ & $0.60 \pm 0.03$ & 1.0 & {$[1.2 \pm 0.2] \times 10^{-4}$} & $0.60 \pm 0.04$ \\
M 87 & $3.02 \pm 0.07$ & $0.52 \pm 0.02$ & 1.0 & {$[0.8 \pm 0.1] \times 10^{-4}$} & $0.50 \pm 0.03$ \\
\hline
\end{tabular}

modeling the kinematic structure of the gas disk (Harms et al. 1994; Macchetto et al. 1997) give us a BH mass of $3.5_{-0.3}^{+0.9} \times$ $10^{9} M_{\odot}$ (Walsh et al. 2013). Therefore, a wide range of SMBH mass estimates exist from $(3.5 \pm 0.8) \times 10^{9} M_{\odot}$ (Walsh et al. $2013)$ to $(6.6 \pm 0.4) \times 10^{9} M_{\odot}$ (Walsh et al. 2013). This agrees with the $\mathrm{BH}$ mass estimate of $7.22_{-0.40}^{+0.34} \times 10^{9} M_{\odot}$ obtained by Oldham \& Auger (2016). In April 2019, the Event Horizon Telescope (EHT) observations released measurements and estimates of a BH mass as $\left(6.5 \pm 0.2_{\text {stat }} \pm 0.7_{\text {sys }}\right) \times 10^{9} M_{\odot}$ (Akiyama et al. 2019b), which is also consistent with the above estimates.

However, it is desirable to use an identification for the core of M 87 that is as independent as possible, as well as a BH mass estimate that uses an alternative to the above-mentioned methods, such as the scaling technique (ST09).

The BH mass scaling process is described in detail in Appendix B. This method can be used to (i) search for such pairs of black holes for which the $\Gamma$ increases with $\dot{M}$ (the BMC normalization, $N_{\mathrm{BMC}}$ ) along the $\Gamma-N_{\mathrm{BMC}}$ track and those for which the saturation level $\Gamma_{\text {sat }}$ is the same, and to (ii) calculate two scaling coefficients which allows determination of the $\mathrm{BH}$ mass of a target.

As can be seen from Figs. 18-20, the correlations of the target sources (3C 454.3 and M 87) and the reference sources are characterized by similar shapes and index saturation levels. Consequently, this method gives us a reliable scaling of these correlations with that of $3 \mathrm{C} 454.3$ and M 87. In order to implement the scaling technique we introduce an analytical approximation of the $\Gamma-N_{\text {bmc }}$ correlation, fitted by Eq. (B.6).

As a result of fitting the observed correlation by this function $F(x)$ (see Eq. (B.6)) we obtained a set of the best-fit parameters $\mathcal{A}, \mathcal{B}, \mathcal{D}, N_{\text {tr }}$, and $\beta$ (see Table 9). The meaning of these parameters is described in detail in our previous papers (Titarchuk \& Seifina 2016b, hereafter TS16; STU18; SCT18). This function $F(x)$ is widely used for a description of the correlation of $\Gamma$ versus $N_{\text {bmc }}$ (Sobolewska \& Papadakis 2009; ST09; Seifina \& Titarchuk 2010; STS14; Giacche et al. 2014; Titarchuk \& Seifina 2016b, 2017; Seifina et al. 2017, 2018b,a).

In order to implement this BH mass determination for the target source one should rely on the same shape of the $\Gamma-N_{\mathrm{BMC}}$ correlations for this target source and those for the reference sources. Accordingly, the only difference in values of $N_{\mathrm{bmc}}$ for these three sources is in the ratio of the $\mathrm{BH}$ mass to the squared distance, $M_{\mathrm{BH}} / d^{2}$. As one can see from Fig. 18 the index saturation value, $\mathcal{A}$, is approximately the same for the target and reference sources (see also the second column in Table 9). The shape of the correlations for 3C 454.3 (violet line) and Cyg X-1 (black line) are similar and the only difference between these correlations is in the BMC normalization values (proportional to $M_{\mathrm{BH}} / d^{2}$ ratio).

To estimate a $\mathrm{BH}$ mass, $M_{\mathrm{t}}$, of $3 \mathrm{C} 454.3$ and M 87 (target sources) one should slide the reference source correlation along the $N_{\mathrm{BMC}}-$ axis to that of the target source (see Figs. 18-20):

$m_{\mathrm{t}}=m_{\mathrm{r}} \frac{N_{\mathrm{t}}}{N_{\mathrm{r}}}\left(\frac{d_{\mathrm{t}}}{d_{\mathrm{r}}}\right)^{2} f_{\mathrm{G}}=m_{\mathrm{r}} \frac{N_{\mathrm{t}}}{N_{\mathrm{r}}}\left(\frac{z_{\mathrm{t}}}{z_{\mathrm{r}}}\right)^{2} f_{\mathrm{G}}$, 
Table 10. Black hole masses and distances.

\begin{tabular}{|c|c|c|c|c|c|}
\hline Source & $M_{\text {dyn }}\left(M_{\odot}\right)$ & $i_{\text {orb }}(\mathrm{deg})$ & $d(\mathrm{kpc})$ & $M_{\text {lum }}\left(M_{\odot}\right)$ & $M_{\text {scal }}\left(M_{\odot}\right)$ \\
\hline GRO J1655-40 & $6.3 \pm 0.3^{(1),(2)}$ & $70 \pm 1^{(1),(2)}$ & $3.2 \pm 0.2^{(3)}$ & $\ldots$ & \\
\hline Cyg X-1 & $6.8-13.3^{(4),(5)}$ & $35 \pm 5^{(4),(5)}$ & $2.5 \pm 0.3^{(4),(5)}$ & $\ldots$ & $7.9 \pm 1.0$ \\
\hline NGC $4051^{(6),(7),(8),(9),(10)}$ & $\ldots$ & $\ldots$ & $\sim 10 \times 10^{3}$ & $\ldots$ & $\geq 6 \times 10^{5}$ \\
\hline NGC $7469^{(11),(12),(13)}$ & $\ldots$ & $\ldots$ & $\sim 70 \times 10^{3}$ & $\ldots$ & $\geq 3 \times 10^{6}$ \\
\hline XTE J1550-564 (14),(15),(16) & $9.5 \pm 1.1$ & $72 \pm 5$ & $\sim 6$ & $\cdots$ & $10.7 \pm 1.5^{(a)}$ \\
\hline H $1743-322^{(17)}$ & $\ldots$ & $75 \pm 3$ & $8.5 \pm 0.8$ & $\ldots$ & $13.3 \pm 3.2^{(a)}$ \\
\hline GRS $1915+105^{(18)}$ & $\ldots$ & $60-70$ & $8.5 \pm 0.8$ & $\ldots$ & $13.3 \pm 3.2^{(a)}$ \\
\hline $4 \mathrm{U} 1630-47^{(19)}$ & $\ldots$ & $\leq 70$ & $\sim 10-11$ & $\ldots$ & $9.5 \pm 1.1$ \\
\hline M 101 ULX-1 (20),(21),(22),(23),(24) & $3-1000$ & $60^{(25)}$ & $\begin{array}{l}(6.4 \pm 0.5) \times 10^{3} \\
(7.4 \pm 0.6) \times 10^{3}\end{array}$ & $\ldots$ & $\geq 3.2 \times 10^{4}, \geq 4.3 \times 10^{4}$ \\
\hline ESO 243-49 HLX-1 (26),(27) & $\ldots$ & $70^{(28)}$ & $\sim 95 \times 10^{3}$ & $8 \pm 4 \times 10^{4}$ & $\geq 7.2 \times 10^{4}$ \\
\hline $3 \mathrm{C} 454.3^{(29)}$ & $\ldots$ & $\leq 4^{(29)}$ & $\sim 30 \times 10^{5}$ & $\ldots$ & $\sim 3.4 \times 10^{9}$ \\
\hline M $87^{(30),(31)}$ & $\ldots$ & $\leq 20^{(31)}$ & $(16.8 \pm 0.8) \times 10^{3}$ & $6.5 \pm 0.7 \times 10^{9}$ & $\sim 5.6 \times 10^{7}$ \\
\hline
\end{tabular}

Notes. ${ }^{(a)}$ Scaling value found by ST09.

References. (1) Greene et al. (2001); (2) Hjellming (1995); (3) Jonker \& Nelemans (2004); (4) Herrero et al. (1995); (5) Ninkov et al. (1987); (6) McHardy et al. (2004); (7) Haba et al. (2008); (8) Pounds \& King (2013); (9) Lobban et al. (2011); (10) Terashima et al. (2009); (11) Peterson et al. (2004); (12) Peterson et al. (2014); (13) Shapovalova et al. (2017); (14) Orosz et al. (2002); (15) Sànchez-Fernàndez et al. (1999); (16) Sobczak et al. (1999); (17) Petri (2008); (18) Mirabel \& Rodrigues (1994); (19) King \& Lasota (2014); (20) STS14; (21) Shappee \& Stanek (2011); (22) Mukai et al. (2005); (23) Kelson et al. (1996); (24) TS15; (25) Liu et al. (2013); (26) Copperwheat et al. (2007); (27) Farrell et al. (2009); (28) Soria (2013); (29) Gupta et al. (2017); (30) Akiyama et al. (2019a); (31) Davis et al. (2011).

where $m_{\mathrm{t}}=M_{\mathrm{t}} / M_{\odot}, m_{\mathrm{r}}=M_{\mathrm{r}} / M_{\odot}$ are the dimensionless $\mathrm{BH}$ masses with respect that of the sun, and $z_{\mathrm{r}}, z_{\mathrm{t}}$ are redshifts of the reference and target sources, correspondingly.

In Fig. 18 we demonstrate the $\Gamma-N_{\text {bmc }}$ correlation for 3C 454.3 (violet points) obtained using the RXTE spectra along with the correlations for the two Galactic reference sources (GRO J1655-40 (pink), Cygnus X-1 (black)) and two extragalactic reference source (NGC 4051 (green line) and NGC 7469 (blue line)), which are similar to the correlation found for the target source.

For M87, we performed scaling with both galactic and extragalactic sources. Specifically, Fig. 19 presents $\Gamma$ versus normalization, $N_{\mathrm{BMC}}$ for M 87 (black line - target source) along with $\Gamma$-Norm correlations for extragalactic sources, ESO 243-49 HLX-1 and M 101 ULX-1 (reference sources). It can be seen that the Chandra points go to the left with respect of other points, perhaps due to the fact that we applied a more compact angular region around the nucleus of M 87 for the Chandra spectra. Figure 20 shows the photon index $\Gamma$ versus the normalization $N_{\mathrm{BMC}}$ for M 87 along with $\Gamma-N_{\text {BMC }}$ correlations for four Galactic reference sources: XTE J1550-564, 4U 1630-472 and H 1743-322. The black line indicates the $\Gamma-N_{\mathrm{BMC}}$ correlation corrected for a nucleus emission from M 87 using the Chandra data. For an estimate of a BH mass in $\mathrm{M} 87$ we use this corrected $\Gamma-N_{\mathrm{BMC}}$ track. The $\mathrm{BH}$ masses and distances for each of these target-reference pairs are shown in Table 10.

We apply values of $m_{\mathrm{r}}, m_{\mathrm{t}}, d_{\mathrm{r}}, d_{\mathrm{t}}$, and $\cos (i)$ (see Table 10) and then we calculate the $\mathrm{BH}$ mass for $3 \mathrm{C} 454.3$ using the bestfit value of $N_{\mathrm{t}}^{3 \mathrm{c}}=(1.2 \pm 0.1) \times 10^{-4}$ taken at the beginning of the index saturation (see Fig. 18) and measured in units of $L_{39} / d_{10}^{2} \mathrm{erg} \mathrm{s}^{-1} \mathrm{kpc}^{-2}$ (see Table 9 for values of the parameters of function $F\left(N_{\mathrm{t}}\right)(\mathrm{Eq} .(1))$.

For M 87, the best-fit value is $N_{\mathrm{t}}^{\mathrm{m} 87}=(0.8 \pm 0.1) \times 10^{-4}$. Using $d_{\mathrm{r}}, m_{\mathrm{r}}, N_{\mathrm{r}}$ (see ST09) we found that $C_{0} \sim 2.0,2.0,1.9$, and 1.83 for NGC 7469, NGC 4051, GRO J1555-40, and Cyg X-1, respectively (for $3 \mathrm{C} 454.3$ case). Similarly, we obtained that $C_{0} \sim 1.9,2.0,2.1$, and 2.03 for XTE J1550-564, H 1743-322, GRS $1915+105$ and 4U 1630-472, respectively.
We find that $M_{3 \mathrm{C}} \geq 3.4 \times 10^{9} M_{\odot}\left(M_{3 \mathrm{C}}=M_{\mathrm{t}}\right)$ assuming $d_{3 \mathrm{C}} \sim$ $3 \times 10^{3}$ Mpc (Gupta et al. 2017; Wright 2006). To determine the distance to $3 \mathrm{C} 454.3$ we used the formula

$d_{3 \mathrm{c}}=z_{3 \mathrm{c}} c / H_{0}$,

where the redshift $z_{3 \mathrm{c}}=0.859$ for $3 \mathrm{C} 454.3$ (see Wright 2006), $c$ is the speed of light, and $H_{0}=70.8 \pm 1.6 \mathrm{~km} \mathrm{~s}^{-1} \mathrm{Mpc}^{-1}$ is the Hubble constant. For M 87 case, we obtain $M_{87} \geq 5.6 \times 10^{7} M_{\odot}$ $\left(M_{87}=M_{\mathrm{t}}\right)$ assuming $d_{\mathrm{m} 87} \sim 16.8 \mathrm{Mpc}$ (Akiyama et al. 2019b).

It is worth noting that for M 87 , we take into account the correction (dashed line) of the $\Gamma$-Normalization of the track. This correction for the jet contamination is important in the case of M 87. In fact, for a reliable BH mass estimate we need to use the nucleus emission to scale the $\Gamma-N_{\mathrm{BMC}}$ correlations. In addition, we selected reference sources with a well-known geometric factor (i.e., the angles at which we see the source). This allowed us to obtain a more accurate estimate of the $\mathrm{BH}$ mass and not just its lower limit. It should also be emphasized that the BH mass value in M 87 turned out to be two orders of magnitude smaller than that estimated by standard methods (see e.g., Akiyama et al. 2019b). We summarize all these results in Table 10.

In addition, we tested the possible contribution from the broad-line region (BLR). To disentangle this contribution we investigated the low-energy part of the Chandra spectrum of 3C 454.3 (which was calculated for an angular region that captures only the core) and compared it with the Suzaku spectrum of 3C 454.3 (which captures both the core region and the jet region and the BLR zone). As a result, we find that few photons come from the jet and BLR zone in this range. Therefore, the contribution of BLR is small.

Celotti et al. (2007) argued that blazar spectra are contributed via the bulk Compton process by a cold, relativistic shell of plasma moving (and accelerating) along the jet of a blazar, scattering external photons emitted by the accretion disk and reprocessed in the BLR zone. However, the bulk Comptonization of disk photons provide a spectral component contributing in the far-ultraviolet band, and are therefore currently unobservable. 
On the other hand, the bulk Comptonization of broad-line photons may yield a significant feature in the soft X-ray band. Such a feature is rather transient however, and only dominates over the nonthermal continuum when: (i) the dissipation occurs close to, or within, the BLR; and (ii) other competing processes, like the synchrotron self-Compton emission, yield a negligible flux in the X-ray band. The presence of a bulk Compton component may account for the X-ray properties of high-redshift blazars that show a flattening (and possibly a hump) in the soft X-rays, previously interpreted as due to intrinsic absorption.

We also tested Suzaku and Chandra data and concluded that the spectra of $3 \mathrm{C} 454.3$ are even less influenced by jet. In fact, the Chandra spectra focused on a compact area around the nucleus of 3C 454.3 (without the jet contamination), while Suzaku spectra come from some wider zone (nucleus plus jet and outer parts of the AGN). We analyzed and compared these Suzaku and Chandra spectra. As a result, we concluded that the jet contribution $(\mathrm{SSC}+\mathrm{CE})$ is too low for the observations used in the paper.

The obtained $\mathrm{BH}$ mass estimate is in agreement with a high bolometric luminosity for $3 \mathrm{C} 454.3$ and the $k T_{\mathrm{s}}$ value which is in the range of $100-260 \mathrm{eV}$ using the Suzaku and Chandra spectra. For example, Shakura \& Sunyaev (1973) and Novikov \& Thorne (1973) provide an effective temperature of the accretion material, $T_{\text {eff }} \sim T_{\mathrm{s}} \propto M_{\mathrm{BH}}^{-1 / 4}$.

It is also important to emphasize that our $\mathrm{BH}$ mass estimate in $3 \mathrm{C} 454.3$ is consistent with a SMBH mass of $(0.5-1.5) \times$ $10^{9} M_{\odot}$ (Gupta et al. 2017; Woo \& Urry 2002; Liu et al. 2006; Sbarrato et al. 2012; Gu et al. 2001). The derived BH mass is the lower limit estimate only because the photon index versus the normalization has the uncertainty with geometrical factor $f_{\mathrm{G}}=\left(\cos i_{\mathrm{d}}\right)_{\mathrm{r}} /\left(\cos i_{\mathrm{d}}\right)_{\mathrm{t}}$. Generally, the photon index versus the QPO frequency correlation enables us to obtain the precise $\mathrm{BH}$ mass (see ST09). Although our BH mass estimate is only the lower limit of that, it significantly constrains the $\mathrm{BH}$ mass for 3C 454.3 (see Table 10).

We derived the bolometric luminosity using the normalization of the BMC model and found it to be between $2 \times 10^{46} \mathrm{erg} \mathrm{s}^{-1}$ and $4 \times 10^{47} \mathrm{erg} \mathrm{s}^{-1}$ (assuming isotropic radiation). Observations of many Galactic BHs (GBHs) and their X-ray spectral analysis (see ST09, Titarchuk \& Seifina 2009; Seifina \& Titarchuk 2010 and STS14, SCT18, STU18) confirm the TZ98 prediction that the spectral (photon) index saturates with the mass accretion rate, the latter being related to the Eddington ratio. This value is not so far from the Eddington limit for the obtained BH mass and assumed source distance (see Table 10).

The resulting mass of the central $\mathrm{BH}$ in $3 \mathrm{C} 454.3$ is in agreement with previous estimates of a BH mass Gupta et al. (2017), Woo \& Urry (2002), Liu et al. (2006), Sbarrato et al. (2012), $\mathrm{Gu}$ et al. (2001). At the same time its high value raises the question of the origin of such SMBHs and their high variability. The answer to this question can be given by a timing analysis using the longest interval of observations. There are indications of a duality of the central object (Volvach et al. 2017), which may partly manifest itself in the form of a high variability of its radiation and; updated scenarios and justification for feeding such a system of two BHs are however needed. Further observations of 3C 454.3 in a different range of frequencies could shed light on these and other problems.

\section{Conclusions}

We found the spectral state transitions observed in 3C 454.3 and M 87 using the full set of Suzaku (2007-2010), Swift (20052009), RXTE (1996-2010), Chandra, ASCA, and BeppoSAX data and demonstrated a validity of the spectral BMC model for fitting the observed spectra for all these sets of observations, independently of the spectral state of the sources.

We investigated the X-ray flaring properties of 3C 454.3 and M 87 and revealed spectral state transitions during the outbursts using the index-normalization (proportional to $\dot{M}$ ) correlation observed in 3C 454.3 and M 87, which were similar to those in Galactic BHs as well as in a number of extragalactic BH sources. In particular, we find that $3 \mathrm{C} 454.3$ follows the $\Gamma-\dot{M}$ correlation previously obtained for extragalactic SMBH sources, NGC 4051 (SCT18), NGC 7469 (STU18), Galactic BHs GROJ1655-40, and Cyg X-1 (ST09) for which we take into account the particular values of the $M_{\mathrm{BH}} / d^{2}$ ratio (see Fig. 18). We find that M 87 follows the $\Gamma-\dot{M}$ correlation previously obtained for extragalactic IMBHs, ESO 243-49 HLX-1 (ST16a) and M 101 ULX-1 (TS16b), and Galactic BHs, XTEJ1550-564, 4U 1630-472, GRS 1915+105, and H 1743-322 (ST09) which we use as reference sources, for which we take into account the particular values of the $M_{\mathrm{BH}} / d^{2}$ ratio (see Figs. 19 and 20).

The photon index $\Gamma$ of the $3 \mathrm{C} 454.3$ spectra is in the range of $\Gamma=1.2-2.1$, while the photon index $\Gamma$ of the M 87 spectra varies in the range of $\Gamma=1.3-3.1$. We tested a possible effect of the presence of the jet for 3C 454.3 and M 87 on our results using Chandra observations. We conclude that the jet contribution in the spectrum is relatively small in the case of $3 \mathrm{C} 454.3$, but can contribute to the M 87 spectra. Therefore, to estimate the $\mathrm{BH}$ mass for $\mathrm{M} 87$, it was crucial to separate the radiation of the nucleus and the jet.

We also find that the peak bolometric luminosity for 3C 454.3 is about $2 \times 10^{46} \mathrm{erg} \mathrm{s}^{-1}$. We use the observed index-mass accretion rate correlation to estimate $M_{\mathrm{BH}}$ in 3C 454.3. This scaling method was successfully applied to find BH masses of Galactic (e.g. ST09, STF13) and extragalactic BHs (TS16; Sobolewska \& Papadakis 2009; Giacche et al. 2014; Titarchuk \& Seifina 2017; Seifina et al. 2017, 2018b,a). Applying the scaling technique to the X-ray data for Suzaku, Swift, Chandra, ASCA, BeppoSAX, and RXTE observations of $3 \mathrm{C} 454.3$ and $\mathrm{M} 87$ allows us to estimate $M_{\mathrm{BH}}$ for these particular sources. We find values of $M_{\mathrm{BH}} \sim 3.4 \times 10^{9} M_{\odot}$ (for $3 \mathrm{C} 454.3)$ and $M_{\mathrm{BH}} \sim 5.6 \times 10^{7} M_{\odot}($ for M 87$)$.

It must also be emphasized that the mass of the $\mathrm{BH}$ in $\mathrm{M} 87$ estimated in this paper $\left(M_{\mathrm{BH}}^{\mathrm{m} 87} \sim 5.6 \times 10^{7} M_{\odot}\right)$ turned out to be two orders of magnitude smaller than that estimated by standard methods (Gebhardt \& Thomas 2009; Gebhardt et al. 2011; Akiyama et al. 2019b) that indicate a $\mathrm{BH}$ mass for $\mathrm{M} 87$ of $\geq 6 \times 10^{9} M_{\odot}$. This discrepancy in BH mass estimates for M 87 poses a problem to observers and theorists. However, the power spectrum lead us to lower the BH mass in M 87 (see Sect. 4.1).

Our BH mass estimate for $3 \mathrm{C} 454.3$ on the other hand is in agreement with the previous $\mathrm{BH}$ mass evaluations of $0.5-4.5 \times$ $10^{9} M_{\odot}$ derived using different $\mathrm{BH}$ mass estimate methods and multiwavelength observations (Gupta et al. 2017; Woo \& Urry 2002; Liu et al. 2006; Sbarrato et al. 2012; Gu et al. 2001). Combining all these estimates with the inferred low temperatures of the seed disk photons $k T_{\mathrm{s}}$ we establish that the compact object of $3 \mathrm{C} 454.3$ is likely to be a SMBH with at least $M_{\mathrm{BH}}^{3 \mathrm{c}}>$ $3.4 \times 10^{9} M_{\odot}$.

Acknowledgements. This research was performed using data supplied by the UK Swift Science Data Centre at the University of Leicester. We also used the data from the GLAST-AGILE Support program at www.oato.inaf.it/ blazars/webt/. This paper has made use of up-to-date SMART optical/nearinfrared light curves that are available at www.astro.yale.edu/smarts/ glast/home.php. We also acknowledge the deep analysis of the paper by the referee. We thank Lubov Ugolkova for useful discussion on the subject of the presented paper. 


\section{References}

Abdo, A. A., Ackermann, M., Ajello, M., et al. 2009, ApJ, 699, 817 Abdo, A. A., Ackermann, M., Ajello, M., et al. 2010, ApJ, 716, 835 Abramowski, A., Acero, F., Aharonian, F., et al. 2012, ApJ, 746, 151

Akiyama, K., Tazaki, F., Moriyama, K., et al. 2019a, Astrophysics Source Code Library [record ascl:1904.005]

Akiyama, K., Alberdi, A., Alef, W., et al. 2019b, ApJ, 875, L6

Bird, S., Harris, W. E., Blakeslee, J. P., \& Flynn, C. 2010, A\&A, 524, A71

Blakeslee, J. P., Jordan, A., Mei, S., et al. 2009, ApJ, 694, 556

Boella, G., Chiappetti, L., Conti, G., et al. 1997, A\&AS, 122, 327

Bonning, E. W., Bailyn, C., Urry, C. M., et al. 2009, ApJ, 697, L81

Bradt, H. V., Rothschild, R. E., \& Swank, J. H. 1993, A\&AS, 97, 533

Cantiello, M.,Blakeslee, J. P., Ferrarese, L., et al. 2018, ApJ, 856, 126

Chitnis, V. R., Pendharkar, J. K., Bose, D., et al. 2009, ApJ, 698, 1207

Celotti, A., Ghisellini, G., \& Fabian, A. C. 2007, MNRAS, 375, 417

Copperwheat, Ch., Cropper, M., Soria, R., \& Wu, K. 2007, MNRAS, 376, 1407

Davis, S.W., Narayan, R., Zhu, Y., et al. 2011, ApJ, 734, 111

Donnarumma, I., Pucella, G., Vittorini, V., et al. 2008, ATel, 1625, 1

Donnarumma, I., Pucella, G., Vittorini, V., et al. 2009, ApJ, 707, 1115

Evans, P. A., Beardmore, A. P., Page, K. L., et al. 2007, A\&A, 469, 379

Evans, P. A., Beardmore, A. P., Page, K. L., et al. 2009, MNRAS, 397, 1177

Farrell, S. A., Webb, N. A., Barret, D., Godet, O., \& Rodrigues, J. M. 2009, Nature, 460, 73

Frontera, F., Costa, E., dal Fiume, D., et al. 1997, Proc. SPIE, 3114, 206

Gebhardt, K., \& Thomas, J. 2009, ApJ, 700, 1690

Gebhardt, K., Adams, J., Richstone, D., et al. 2011, ApJ, 729, 119

Giacche, S., Gili, R., \& Titarchuk, L. 2014, A\&A, 562, A44

Giommi, P., Blustin, A. J., Capalbi, M., et al. 2006, A\&A, 456, 911

Greene, J., Bailyn, C. D., \& Orosz, J. A. 2001, ApJ, 554, 1290

Gu, M., Cao, X., \& Jiang, D. R. 2001, MNRAS, 327, 1111

Gupta, A. C., Mangalam, A., Wiita, P. J., et al. 2017, MNRAS, 472, 788

Haba, Y., Liebmann, A. C., \& Fukumura, K. 2008, PASJ, 60, 1257

Harms, R. J., Ford, H., Tsvetanov, Z. I., et al. 1994, ApJ, 435, L35

Herrero, J., Kudritzki, R. P., Gabler, R., et al. 1995, A\&A, 297, 556

Harris, D. E., Cheung, C. C., Stawarz, L., Biretta, J. A., \& Perlman, E. S. 2009, ApJ, 699, 305

Hjellming, R. M., Rupen, M. P. 1995, Nature, 375, 464

Hovatta, T., Valtaoja, E., Tornikoski, M., \& Lahteenmaki, A. 2009, A\&A, 494, 527

Hubble, E. P. 1926, ApJ, 64, 321

Jackson, N., \& Browne, I. W. A. 1991, MNRAS, 250, 422

Jonker, P. G., \& Nelemans, G. 2004, MNRAS, 354, 355

Jorstad, S. G., Marscher, A. P., Mattox, J. R., et al. 2005, AJ, 130, 1418

Jorstad, S., Marscher, A., Larionov, V., et al. 2010, ApJ, 715, 362

Kardashev, N. 1962, Sov. Astron., 6, 317

King, A., \& Lasota, J.-P. 2014, MNRAS, 444, L30

Kelson, D. D., Illingworth, G. D., Saha, A., et al. 1996, ApJ, 463, 26

Koyama, K., Tsunemi, H., Dotani, T., et al. 2007, PASJ, 59, 23

Kellermann, K. I., Vermeulen, R. C., Zensus, J. A., et al. 1998, AJ, 115, 1295

Kennea, J., Boehringer, H., Belsole, E., et al. 2000, in American Astronomical Society, 197th AAS Meeting, id.79.03, BAAS, 32, 1532

Khangulyan, D. V., Barkov, M. V., Bosch-Ramon, V., Aharonian, F. A., \& Dorodnitsyn, A. V. 2013, ApJ, 774, 113

Laurent, P., \& Titarchuk, L. 1999, ApJ, 511, 289

Laurent, P., \& Titarchuk, L. 2007, ApJ, 656, 1056

Laurent, P., \& Titarchuk, L. 2011, ApJ, 727, 34

Liu, Y., Jiang, D. R., \& Gu, M. F. 2006, ApJ, 637, 669

Liu, J., Bregman, J. N., Bai, Y., et al. 2013, Nature, 503, 500

Lobban, A. P., Reeves, J. N., \& Miller, L. 2011, MNRAS, 414, 1965

Macchetto, F., Marconi, A., Axon, D. J., et al. 1997, ApJ, 489, 579

Massaro, E., Perri, M., Giommi, P., \& Nesci, R. 2004a, A\&A, 413, 489

Massaro, E., Perri, M., Giommi, P., et al. 2004b, A\&A, 422, 103

Massaro, E., Tramacere, A., Perri, M., Giommi, P., \& Tosti, G. 2006, A\&A, 448, 861

McHardy, I. M., Papadakis, I. E., Uttley, P., et al. 2004, MNRAS, 348, 783

Mirabel, I. F., \& Rodrigues, L. F. 1994, Nature, 371, 46

Mitsuda, K., Bautz, M., Inoue, H., et al. 2007, PASJ, 59, S1

Mukai, K., Still, M., Corbet, R., Kuntz, K., \& Barnard, R. 2005, ApJ, 634, 1085

Ninkov, Z., Walker, G. A. H., \& Yang, S. 1987, ApJ, 321, 425

Novikov, I. D., \&amp;amp; Thorne, K. S. 1973, in Black holes (Les astres occlus), eds. C. DeWitt, \& B. DeWitt (NY: Gordon and Breach), 343
Oldham, L. J., \& Auger, M. W. 2016, MNRAS, 457, 421

Orosz, J. A., Groot, P. J., van der Klis, M., et al. 2002, ApJ, 568, 84

Pacciani, L., Vittorini, V., Tavani, M., et al. 2010, ApJ, 716, L170

Parmar, A. N., Williams, O. R., Kuulkers, E., Angelini, L., \& White, N. E. 1997, A\&A, 319, 855

Pounds, K. A., \& King, A. R. 2013, MNRAS, 433, 1369

Peterson, B. M., Ferrarese, L., Gilbert, K. M., et al. 2004, ApJ, 613, 682

Peterson, B. M., Grier, C. J., Horne, K., et al. 2014, ApJ, 795, 149

Petri, J. 2008, Ap\&SS, 318, 181

Pian, E., Foschini, L., Beckmann, V., et al. 2006, A\&A, 449, 21

Raiteri, C. M., Villata, M., Lanteri, L., Cavallone, M., \& Sobrito, G. 1998, A\&AS, 130, 495

Raiteri, C. M., Villata, M., Larionov, V. M., et al. 2007, A\&A, 473, 819

Raiteri, C. M., Villata, M., Chen, W. P., et al. 2008a, A\&A, 485, L17

Raiteri, C. M., Villata, M., Larionov, V. M., et al. 2008b, A\&A, 491, 755

Raiteri, C. M., Villata, M., Aller, M. F., et al. 2011, A\&A, 534, A87

Rivers, E., Markowitz, A., \& Rothschild, R. E. 2011, ApJS, 193, 3

Rivers, E., Markowitz, A., \& Rothschild, R. 2013, ApJ, 772, 114

Rybick, G. B., \& Lightman, A. P. 1979, Radiative Processes in Astrophysics (John Wiley \& Sons, Inc)

Sànchez-Fernàndez, C., Castro-Tirado, A. J., Duerbeck, H. W., et al. 1999, A\&A, 348, L9

Sasada, M., Uemura, M., Fukazawa, Y., et al. 2010, PASJ, 62, 645

Sbarrato, T., Ghisellini, G., Maraschi, L., \& Colpi, M. 2012, MNRAS, 421, 1764

Seifina, E., \& Titarchuk, L. 2010, ApJ, 722, 586 (ST10)

Seifina, E., Titarchuk, L., \& Shaposhnikov, N. 2014, ApJ, 789, 57

Seifina, E., Titarchuk, L., \& Virgilli, E. 2017, A\&A, 607, A38

Seifina, E., Titarchuk, L., \& Ugolkova, L. 2018a, A\&A, 619, A21

Seifina, E., Chekhtman, A., \& Titarchuk, L. 2018b, A\&A, 613, A48

Shakura, N. I., \& Sunyaev, R. A. 1973, A\&A, 24, 337

Shaposhnikov, N., \& Titarchuk, L. 2009, ApJ, 699, 453

Shaposhnikov, N., Swank, J., Shrader, C. R., Rupen, M., et al. 2007, ApJ, 655, 434

Shapovalova, A. I., Popovic, L. C., Chavushyan, V. H., et al. 2017, MNRAS, 466,4759

Shappee, B., \& Stanek, K. Z. 2011, ApJ, 733, 124

Shrader, Ch., \& Titarchuk, L. 1998, ApJ, 499, L31

Sobczak, G. J., McClintock, J. E., Remillard, R. A., \& Bailyn, C. D. 1999, ApJ, 520,776

Sobolewska, M. A., \& Papadakis, I. E. 2009, MNRAS, 399, 1997

Soria, R. 2013, MNRAS, 428, 1944

Tanaka, Y., Inoue, H., \& Holt, S. S. 1994, PASJ, 46, L37

Terashima, Y., Gallo, L. C., \& Inoue, H. 2009, PASJ, 61, S299

Titarchuk, L., \& Seifina, E. 2009, ApJ, 706, 1463

Titarchuk, L., \& Seifina, E. 2016a, A\&A, 595, A110

Titarchuk, L., \& Seifina, E. 2016b, A\&A, 585, A94

Titarchuk, L., \& Seifina, E. 2017, A\&A, 602, A113 (TS17)

Titarchuk, L., \& Zannias, T. 1998, ApJ, 499, 315

Titarchuk, L., Mastichiadis, A., \& Kylafis, N. D. 1997, ApJ, 487, 834

Titarchuk, L., Lapidus, I. I., \& Muslimov, A. 1998, ApJ, 499, 315

Unwin, S. C., Wehrle, A. E., Lobanov, A. P., et al. 1997, ApJ, 480, 596

Urry, C. M., \& Padovani, P. 1995, PASP, 107, 803

Vercellone, S., Chen, A. W., Giuliani, A., et al. 2008, ApJ, 676, L13

Vercellone, S., Chen, A. W., Vittorini, V., et al. 2009, ApJ, 690, 1018

Vercellone, S., D'Ammando, F., Vittorini, V., et al. 2010, ApJ, 712, 405

Vestergaard, M., \& Osmer, P. S. 2009, ApJ, 699, 800

Vaughan, S., Goad, M. R., Beardmore, A. P., et al. 2006, ApJ, 63, 920

Villata, M., Raiteri, C. M., Balonek, T. J., et al. 2006, A\&A, 453, 817

Villata, M., Raiteri, C. M., Gurwell, M. A., et al. 2009, A\&A, 504, L9

Volvach, A. E., Volvach, L. N., Larionov, M. G., et al. 2008, Astron. Rep., 52, 867

Volvach, A. E., Volvach, L. N., Kutkin, A. M., et al. 2010, Astron. Rep., 54, 28

Volvach, A. E., Volvach, L. N., Kutkin, A. M., et al. 2011, Astron. Rep., 55, 608

Volvach, A. E., Kutkin, A. M., Volvach, L. N., et al. 2013, Astron. Rep., 57, 34

Volvach, A. E., Larionov, M. G., Volvach, L. N., Larionov, G. M., et al. 2017, Astron. Rep., 61, 954

Walsh, J. L., Barth, A. J., Ho, L. C., \& Sarzi, M. 2013, ApJ, 770, 86

Wilson, A. S., \& Yang, Y. 2002, ApJ, 568, 133

Woo, J.-H., \& Urry, C. M. 2002, ApJ, 579, 530

Wright, E. L. 2006, PASP, 118, 1711

Zamaninasab, M., Savolainen, T., Clausen-Brown, E., et al. 2013, MNRAS, 436, 3341 


\section{Appendix A: Comptonized spectrum using the BMC model}

The BMC model describes the outgoing spectrum as a convolution of the input "seed" blackbody-like spectrum, whose normalization is $N_{\mathrm{BMC}}$ and color temperature is $k T$, with the Comptonization Green's function. It was realized a long time ago (see e.g., Titarchuk et al. 1997; Shrader \& Titarchuk (1998); Laurent \& Titarchuk 1999, hereafter LT99) that one should compute the expected spectral energy distribution for an accreting $\mathrm{BH}$ and neutron star binaries in terms of only four model parameters: the disk color temperature, a geometric factor related to the illumination of the $\mathrm{BH}$ site by the disk, a spectral index related to the efficiency of the Comptonization (thermal or bulk-motion upscattering), and the normalization (the so-called BMC model). In this case the shape of the emergent spectrum is described by Eq. (3). If we want take into account the recoil effect we should introduce one more parameter $E_{\mathrm{cf}}$ which characterizes the highenergy cutoff energy (see the COMPTT or COMPTB models). The BMC analytical model was successfully checked by LT99 and Laurent \& Titarchuk (2011) using the Monte Carlo simulations. These latter authors demonstrated that the core of the spectrum, until the recoil high-energy cut-off, is exactly described by the BMC model.

\section{Appendix B: The $\Gamma$-normalization correlation and details of the $\mathrm{BH}$ mass estimates}

The evaluation method of a black holes mass is based on a comparative analysis of similar $\Gamma-N_{\mathrm{BMC}}$ tracks by scaling their characteristics. It was shown in Titarchuk et al. (1998) and LT99 that the photon index $\Gamma$ monotonically increases with an increase in the accretion rate (which is proportional to the normalization parameter in the Compotonization model; e.g. COMPTT, COMPTB, BMC) and saturates, i.e., reaches a constant level, with large values of the mass accretion rate $\dot{M}$. The steepness for the $\Gamma-N_{\mathrm{BMC}}$ track and the saturation level of $\Gamma_{\text {sat }}$ may be different for different black holes. The comparative analysis in the scaling problem is therefore simplified as searching for such pairs of black holes for which the rate of $\Gamma$ increases with $\dot{M}$ $\left(N_{\mathrm{BMC}}\right)$ along the $\Gamma-N_{\mathrm{BMC}}$ track and the saturation level $\Gamma_{\text {sat }}$ is the same. It is assumed that for each $\mathrm{BH}$ from such a pair, distances are known. In addition, the mass of one of the black holes of this pair is known. The scaling process itself consists in calculating two scaling coefficients and then with their help the mass of the desired $\mathrm{BH}$ is determined.

We now briefly reiterate the main points of the scaling method. Shaposhnikov et al. (2007, hereafter ST07), apply an inverse proportionality of a frequency of quasi-periodic oscillations (QPO) on BH mass in order to estimate the latter. ST07 also present theoretical arguments in terms of the transition layer model for the inverse proportionality of QPO frequencies on BH mass. Therefore, a first scaling law claims that

$s_{v}=\frac{v_{\mathrm{r}}}{v_{\mathrm{t}}}=\frac{M_{\mathrm{t}}}{M_{\mathrm{r}}}$

where subscript $r$ and $t$ subscripts denote reference and target sources.

The second scaling law, which we use as a basis for our scaling technique, is the detected intensity dependence of the source luminosity and distance:

$$
\frac{F_{\mathrm{r}}}{F_{\mathrm{t}}}=\frac{L_{\mathrm{r}}}{L_{\mathrm{t}}} \frac{d_{\mathrm{t}}^{2}}{d_{\mathrm{r}}^{2}} .
$$

Here, $F$ stands for the source flux detected by an observer on Earth, $L$ is the source luminosity, and $d$ is a source distance. The luminosity $L$ can be represented as

$L=\frac{G M_{\mathrm{BH}}}{R_{*}} \dot{M} \eta \sim \frac{G M_{\mathrm{BH}}}{R_{\mathrm{S}}} \dot{M} \eta \sim \dot{M} \eta=M_{\mathrm{BH}} \dot{m} \eta$,

where $R_{*}$ is the effective radius where energy release occurs, $\eta$ is the efficiency of gravitation energy conversion into radiation power, $\dot{M}$ is the accretion rate, and $\dot{m}$ is its dimensionless ana$\log$ normalized by the Eddington luminosity. Both $\dot{m}$ and $\eta$ are considered to be the same for two different sources and for the same spectral state, which lead us to $L_{\mathrm{r}} / L_{\mathrm{t}}=M_{\mathrm{r}} / M_{\mathrm{t}}=1 / s_{v}$. In our analysis of energy spectra from BHs we determine normalization of seed photon radiation supplied by an accretion flow (disk) prior to Comptonization. The ratio of this normalization for the two sources in the same spectral state can be written as

$s_{\mathrm{N}}=\frac{N_{\mathrm{r}}}{N_{\mathrm{t}}}=\frac{L_{\mathrm{r}}}{L_{\mathrm{t}}} \frac{d_{\mathrm{t}}^{2}}{d_{\mathrm{r}}^{2}} f_{\mathrm{G}}$

Here, $f_{\mathrm{G}}$ is a geometry factor that comes due to the fact that the accretion disk which produces thermal input for Comptonization has a plane geometry. Therefore, in the case of radiation coming directly from the disk it would have the value $f_{\mathrm{G}}=\left(\cos i_{\mathrm{d}}\right)_{\mathrm{r}} /\left(\cos i_{\mathrm{d}}\right)_{\mathrm{t}}$, where $i_{\mathrm{d}}$ is the inclination angle of the disk. When information on a (target and reference) source inclination is available we can use the values $i_{\mathrm{d}, \mathrm{r}}$ and $i_{\mathrm{d}, \mathrm{t}}$.

We are now ready to write down the final equations of our scaling analysis. When $s_{\mathrm{N}}$ are measured directly using observations, the mass of the target source can be calculated as

$m_{\mathrm{t}}=m_{\mathrm{r}} \frac{d_{\mathrm{t}}^{2}}{d_{\mathrm{r}}^{2}} \frac{f_{\mathrm{G}}}{s_{\mathrm{N}}}$.

This equation allows estimation of a ratio $m_{\mathrm{t}}$ using values of $m_{\mathrm{r}}, s_{\mathrm{N}}, f_{\mathrm{G}}, d_{\mathrm{t}}$ and $d_{\mathrm{r}}$.

Using Eq. (B.5), the problem of BH mass and distance measurements for a target source is reduced to the determination of scaling coefficients $s_{\mathrm{N}}$ with respect to the data for a reference source. This can be obtained by a technique similar to that adopted by ST07. Specifically, after scalable state transition episodes are identified for two sources, the correlation pattern for a reference transition is parameterized in terms of the analytical function (see also ST09),

$F(x)=\mathcal{A}-(\mathcal{D} \cdot \mathcal{B}) \ln \left\{\exp \left[\left(1.0-\left(x / x_{\text {tr }}\right)^{\beta}\right) / \mathcal{D}\right]+1\right\}$,

where $x=N_{\mathrm{bmc}}$.

After rearrangement, the $\mathrm{BH}$ mass $M_{\mathrm{t}}$ of a target source can be evaluated using the formula (see TS16)

$m_{\mathrm{t}}=C_{0} N_{\mathrm{t}} d_{\mathrm{t}}^{2} f_{\mathrm{G}}$,

where $C_{0}=\left(1 / d_{\mathrm{r}}^{2}\right)\left(m_{\mathrm{r}} / N_{\mathrm{r}}\right)$ is the scaling coefficient for the reference source, $\mathrm{BH}$ masses $m_{\mathrm{t}}$ and $m_{\mathrm{r}}$ are in solar units, and $d_{\mathrm{r}}$ is the distance to a particular reference source measured in kiloparsecs. 
Table B.1. Best-fit parameters of spectral analysis of RXTE observations of 3C 454.3 ( $R 1-R 2$ sets) in the 3-50 keV energy range.

\begin{tabular}{|c|c|c|c|c|c|}
\hline Observational ID & $\begin{array}{l}\text { MJD } \\
\text { (day) }\end{array}$ & $\alpha=\Gamma-1$ & $\begin{array}{l}k T_{\mathrm{s}} \\
(\mathrm{keV})\end{array}$ & $\begin{array}{l}N_{\mathrm{BMC}}{ }^{(\dagger)} \\
\left(L_{35} / d_{10}^{2}\right)\end{array}$ & $\chi_{\text {red }}^{2}$ (d.o.f.) \\
\hline $10360-01-23-00$ & 50358.56 & $0.63 \pm 0.09$ & $0.5 \pm 0.2$ & $1.6 \pm 0.1$ & $0.75(49)$ \\
\hline $10360-01-36-00$ & 50265.65 & $0.54 \pm 0.09$ & $0.5 \pm 0.2$ & $1.0 \pm 0.2$ & $0.87(47)$ \\
\hline $10360-01-27-00$ & 50329.59 & $0.7 \pm 0.1$ & $0.5 \pm 0.2$ & $2.1 \pm 0.2$ & $0.95(47)$ \\
\hline $10360-01-40-00$ & 50240.21 & $0.76 \pm 0.09$ & $0.7 \pm 0.2$ & $2.1 \pm 0.3$ & $0.87(49)$ \\
\hline $10360-01-47-00$ & 50187.57 & $0.3 \pm 0.2$ & $0.5 \pm 0.2$ & $0.4 \pm 0.1$ & $0.85(54)$ \\
\hline $10360-01-48-00$ & 50181.11 & $0.6 \pm 0.2$ & $0.4 \pm 0.4$ & $1.2 \pm 0.3$ & $0.94(54)$ \\
\hline 20346-01-01-00 & 50389.18 & $0.4 \pm 0.2$ & $0.2 \pm 0.1$ & $0.4 \pm 0.1$ & $0.85(54)$ \\
\hline 20346-01-02-00 & 50397.65 & $0.36 \pm 0.07$ & $0.2 \pm 0.1$ & $0.5 \pm 0.1$ & $0.97(54)$ \\
\hline $20346-01-03-00$ & 50406.32 & $0.94 \pm 0.07$ & $0.6 \pm 0.4$ & $2.1 \pm 0.1$ & $0.89(54)$ \\
\hline 20346-01-04-00 & 50411.66 & $0.53 \pm 0.09$ & $0.8 \pm 0.6$ & $1.4 \pm 0.1$ & $0.76(54)$ \\
\hline 20346-01-05-00 & 50419.32 & $0.21 \pm 0.10$ & $0.10 \pm 0.01$ & $0.57 \pm 0.09$ & $1.05(54)$ \\
\hline 20346-01-07-00 & 50430.41 & $0.62 \pm 0.09$ & $0.11 \pm 0.01$ & $0.69 \pm 0.09$ & $1.12(54)$ \\
\hline 20346-01-08-00 & 50437.14 & $0.61 \pm 0.03$ & $0.11 \pm 0.01$ & $0.83 \pm 0.04$ & $0.83(54)$ \\
\hline 20346-01-10-00 & 50453.87 & $0.71 \pm 0.05$ & $0.09 \pm 0.01$ & $1.04 \pm 0.02$ & $0.82(54)$ \\
\hline 20346-01-11-00 & 50461.42 & $0.71 \pm 0.05$ & $0.17 \pm 0.01$ & $1.04 \pm 0.02$ & $0.82(54)$ \\
\hline $20346-01-12-00$ & 50468.77 & $1.01 \pm 0.07$ & $0.12 \pm 0.01$ & $2.56 \pm 0.08$ & $0.95(54)$ \\
\hline 20346-01-14-00 & 50480.36 & $1.00 \pm 0.08$ & $0.20 \pm 0.03$ & $2.82 \pm 0.06$ & $0.93(54)$ \\
\hline $20346-01-15-00$ & 50488.36 & $0.85 \pm 0.02$ & $0.19 \pm 0.01$ & $1.94 \pm 0.09$ & $0.93(54)$ \\
\hline 20346-01-16-00 & 50494.49 & $0.81 \pm 0.02$ & $0.26 \pm 0.02$ & $1.07 \pm 0.07$ & $1.12(54)$ \\
\hline 20346-01-17-00 & 50543.51 & $0.69 \pm 0.08$ & $0.29 \pm 0.06$ & $1.01 \pm 0.09$ & $0.73(54)$ \\
\hline $20346-01-18-00$ & 50551.81 & $0.94 \pm 0.07$ & $0.27 \pm 0.06$ & $5.78 \pm 0.09$ & $0.98(54)$ \\
\hline 20346-01-24-00 & 50594.23 & $0.69 \pm 0.04$ & $0.46 \pm 0.09$ & $1.16 \pm 0.08$ & $0.95(54)$ \\
\hline $20346-01-26-00$ & 50608.24 & $0.13 \pm 0.09$ & $0.31 \pm 0.08$ & $0.40 \pm 0.08$ & $1.00(54)$ \\
\hline 20346-01-27-00 & 50617.22 & $0.93 \pm 0.08$ & $0.20 \pm 0.09$ & $1.56 \pm 0.09$ & $0.73(54)$ \\
\hline $20346-01-28-00$ & 50622.50 & $0.58 \pm 0.03$ & $0.57 \pm 0.08$ & $1.99 \pm 0.05$ & $0.92(54)$ \\
\hline 20346-01-29-00 & 50627.83 & $0.68 \pm 0.06$ & $0.55 \pm 0.09$ & $1.80 \pm 0.06$ & $0.87(54)$ \\
\hline 20346-01-30-00 & 50633.97 & $0.96 \pm 0.05$ & $0.46 \pm 0.04$ & $1.37 \pm 0.09$ & $0.92(54)$ \\
\hline 20346-01-31-00 & 50643.58 & $0.77 \pm 0.06$ & $0.22 \pm 0.07$ & $1.20 \pm 0.07$ & $0.87(54)$ \\
\hline $20346-01-35-00$ & 50673.14 & $0.55 \pm 0.05$ & $0.30 \pm 0.09$ & $1.42 \pm 0.09$ & $0.87(54)$ \\
\hline 20346-01-38-00 & 50693.06 & $0.88 \pm 0.02$ & $0.12 \pm 0.06$ & $1.83 \pm 0.09$ & $0.79(54)$ \\
\hline 20346-01-43-00 & 50725.75 & $1.00 \pm 0.04$ & $0.24 \pm 0.05$ & $3.05 \pm 0.08$ & $0.90(54)$ \\
\hline 20346-01-44-00 & 50733.43 & $0.78 \pm 0.06$ & $0.12 \pm 0.07$ & $1.19 \pm 0.09$ & $0.99(54)$ \\
\hline $20346-01-45-00$ & 50739.89 & $0.72 \pm 0.02$ & $0.12 \pm 0.05$ & $1.12 \pm 0.06$ & $0.95(54)$ \\
\hline $20346-01-46-00$ & 50746.76 & $0.71 \pm 0.08$ & $0.12 \pm 0.05$ & $1.93 \pm 0.08$ & $0.91(54)$ \\
\hline 20346-01-47-00 & 50754.55 & $0.22 \pm 0.02$ & $0.16 \pm 0.05$ & $0.31 \pm 0.06$ & $1.03(54)$ \\
\hline $20346-01-48-00$ & 50765.24 & $0.26 \pm 0.04$ & $0.15 \pm 0.06$ & $0.41 \pm 0.07$ & $1.05(54)$ \\
\hline 20346-01-51-00 & 50783.86 & $1.03 \pm 0.07$ & $0.14 \pm 0.04$ & $2.27 \pm 0.07$ & $0.96(54)$ \\
\hline $20346-01-52-00$ & 50789.39 & $1.04 \pm 0.03$ & $0.10 \pm 0.04$ & $7.30 \pm 0.09$ & $0.97(54)$ \\
\hline $20346-01-53-00$ & 50796.13 & $0.87 \pm 0.03$ & $0.14 \pm 0.03$ & $1.28 \pm 0.09$ & $0.86(54)$ \\
\hline 20346-01-54-00 & 50802.97 & $0.69 \pm 0.08$ & $0.21 \pm 0.04$ & $1.19 \pm 0.09$ & $0.99(54)$ \\
\hline
\end{tabular}

Notes. Parameter errors correspond to $90 \%$ confidence level. The spectral model is tbabs*bmc; where $N_{\mathrm{H}}$ is fixed at a value $5.0 \times 10^{22} \mathrm{~cm}^{-2}(\mathrm{see}$ Sect. 3.2.1); parameter $\log (A)$ is low variable around 1.5. ${ }^{(\dagger)}$ For normalization parameter $N_{\mathrm{BMC}}=L_{35} / d_{10}^{2}$ (see Eqs. (3) and (B.2)) where $L_{35}$ is the source luminosity in units of $10^{35} \mathrm{erg} \mathrm{s}^{-1}$ and $d_{10}$ is the distance to the source in units of $10 \mathrm{kpc}$. 
Table B.2. Best-fit parameters of spectral analysis of RXTE observations of 3C 454.3 ( $R 3-R 5$ sets) in 3-50 keV energy range.

\begin{tabular}{|c|c|c|c|c|c|c|c|}
\hline Observational ID & $\begin{array}{l}\text { MJD } \\
\text { (day) }\end{array}$ & $\alpha=\Gamma-1$ & $\begin{array}{c}k T_{\mathrm{s}} \\
(\mathrm{keV})\end{array}$ & $\log (A)^{(\dagger)}$ & $\begin{array}{l}N_{\mathrm{BMC}}(\dagger \dagger) \\
\left(L_{35} / d_{10}^{2}\right)\end{array}$ & $\chi_{\text {red }}^{2}$ (d.o.f.) & $F_{1} / F_{2}(\dagger+\dagger)$ \\
\hline $30264-01-02-00$ & 50816.05 & $0.94 \pm 0.12$ & $0.38 \pm 0.05$ & $-0.20 \pm 0.04$ & 12.30 .5 & $1.14(46)$ & $35.87 / 23.97$ \\
\hline $30264-01-03-00$ & 50825.97 & $0.53 \pm 0.09$ & $0.42 \pm 0.08$ & $2.00^{(\dagger)}$ & 1.040 .07 & $1.18(48)$ & $9.26 / 8.28$ \\
\hline 30264-01-04-00 & 50831.97 & $0.53 \pm 0.03$ & $0.42 \pm 0.09$ & $2.00^{(\dagger)}$ & 1.030 .09 & $1.16(48)$ & $9.26 / 8.28$ \\
\hline $30264-01-05-00$ & 50839.97 & $0.54 \pm 0.02$ & $0.25 \pm 0.04$ & $2.00^{(\dagger)}$ & 1.060 .09 & $0.95(48)$ & $11.8 / 10.4$ \\
\hline $30264-01-06-00$ & 50846.97 & $0.39 \pm 0.03$ & $0.19 \pm 0.08$ & $2.00^{(\dagger)}$ & 1.050 .08 & $0.95(48)$ & $11.8 / 10.4$ \\
\hline $30264-01-08-00$ & 50860.97 & $0.39 \pm 0.03$ & $0.18 \pm 0.05$ & $2.00^{(\dagger)}$ & 0.660 .05 & $0.98(48)$ & $9.7 / 9.8$ \\
\hline 30264-01-09-00 & 50911.97 & $0.40 \pm 0.05$ & $0.19 \pm 0.07$ & $2.00^{(\dagger)}$ & 0.810 .08 & $0.96(41)$ & $11.87 / 12.0$ \\
\hline $30264-01-10-00$ & 50916.97 & $0.39 \pm 0.01$ & $0.18 \pm 0.03$ & $2.00^{(\dagger)}$ & 0.650 .06 & $0.96(41)$ & $11.86 / 12.1$ \\
\hline $30264-01-12-00$ & 50932.97 & $0.74 \pm 0.03$ & $0.13 \pm 0.02$ & $2.00^{(\dagger)}$ & 0.900 .10 & $0.74(49)$ & $10.05 / 8.45$ \\
\hline $30264-01-13-00$ & 50939.97 & $0.19 \pm 0.02$ & $0.12 \pm 0.02$ & $2.00^{(\dagger)}$ & 0.530 .20 & $1.16(49)$ & $10.9 / 13.2$ \\
\hline 30264-01-14-00 & 50945.97 & $0.18 \pm 0.01$ & $0.12 \pm 0.01$ & $2.00^{(\dagger)}$ & 0.630 .18 & $0.93(49)$ & $13.1 / 15.8$ \\
\hline $30264-01-15-00$ & 50952.97 & $0.88 \pm 0.02$ & $0.20 \pm 0.01$ & $2.00^{(\dagger)}$ & 1.070 .10 & $0.95(48)$ & $12.68 / 8.13$ \\
\hline $30264-01-17-00$ & 50966.97 & $0.89 \pm 0.03$ & $0.20 \pm 0.01$ & $2.00^{(\dagger)}$ & 0.690 .10 & $0.95(48)$ & $12.68 / 8.13$ \\
\hline 30264-01-19-00 & 50980.97 & $0.30 \pm 0.03$ & $0.22 \pm 0.01$ & $2.00^{(\dagger)}$ & 0.410 .09 & $1.01(47)$ & $10.58 / 11.62$ \\
\hline $30264-01-20-00$ & 50986.51 & $0.42 \pm 0.05$ & $0.27 \pm 0.01$ & 2.0 & 0.340 .08 & $0.95(47)$ & $8.4 / 8.29$ \\
\hline $93150-03-01-00$ & 54309.72 & $0.92 \pm 0.06$ & $0.46 \pm 0.04$ & $2.00^{(\dagger)}$ & 7.920 .10 & $1.06(45)$ & $7.05 / 5.21$ \\
\hline 9315 & 54309.93 & $0.95 \pm 0.07$ & $0.47 \pm 0$ & $1.99 \pm 0$ & 7.690 .11 & & 5.06 \\
\hline$-01-02$ & 543 & $0.90 \pm$ & $0.50 \pm$ & $1.66=$ & 6.260 .09 & 45) & $5.72 / 4.02$ \\
\hline 93150-03-01-04 & 54314.60 & $0.98 \pm 0.02$ & $0.46 \pm 0.03$ & $1.95 \pm 0.08$ & 7.410 .10 & $1.13(45)$ & $6.58 / 4.83$ \\
\hline $93150-03-01-06$ & 54313.30 & $0.97 \pm 0.05$ & $0.48 \pm 0.04$ & $1.51 \pm 0.06$ & 7.510 .19 & $1.09(45)$ & $6.77 / 4.80$ \\
\hline $93150-03-02-00$ & 54315.78 & $0.96 \pm 0.08$ & $0.43 \pm 0.05$ & $1.60 \pm 0.07$ & 7.780 .16 & $1.11(45)$ & $6.86 / 5.03$ \\
\hline $93150-03-03-03$ & 54619.15 & $0.96 \pm 0.09$ & $0.44 \pm 0.02$ & $1.59 \pm 0.06$ & 5.310 .15 & 0.72 & $4.67 / 3.42$ \\
\hline 93150-03-03-05 & 54621.02 & $0.95 \pm 0.08$ & $0.43 \pm 0.01$ & $1.63 \pm 0.04$ & 3.530 .17 & $0.95(45)$ & $3.10 / 2.29$ \\
\hline $93150-03-04-00$ & 54624.36 & $0.91 \pm 0.07$ & $0.38 \pm 0.02$ & $1.76 \pm 0.05$ & 3.570 .16 & $0.97(45)$ & $3.05 / 2.34$ \\
\hline $93150-03-04-01$ & 54623.85 & $0.89 \pm 0.06$ & $0.28 \pm 0.04$ & $1.61 \pm 0.09$ & 3.270 .18 & $0.79(45)$ & $2.64 / 2.18$ \\
\hline $93150-03-04-02$ & & & & & & & \\
\hline $93150-03-04-04$ & 54627.44 & $0.90 \pm$ & $0.35 \pm 0.02$ & $0.99 \pm 0.08$ & 4.140 .13 & $0.98(45)$ & $3.54 / 2.54$ \\
\hline 93150-03-04-05 & 54628.12 & $0.94 \pm 0.07$ & $0.26 \pm 0.03$ & $1.05 \pm 0.09$ & 4.480 .19 & $0.94(45)$ & $3.65 / 2.86$ \\
\hline $93150-03-05-02$ & 54632.34 & $0.92 \pm 0.06$ & $0.34 \pm 0.02$ & $0.89 \pm 0.08$ & 3.820 .18 & $0.96(45)$ & $3.29 / 2.31$ \\
\hline $93150-03-05-04$ & 54634.58 & $0.91 \pm 0.08$ & $0.43 \pm 0.07$ & $0.81 \pm 0.06$ & 3.220 .19 & $0.98(45)$ & $2.93 / 1.88$ \\
\hline $93150-03-05-05$ & 54635.18 & $0.92 \pm 0.09$ & $0.36 \pm 0$ & $0.85 \pm 0.02$ & 3.270 .13 & $0.96(45)$ & $2.87 / 1.95$ \\
\hline $93150-03-05-06$ & 54636.81 & $0.93 \pm 0.08$ & $0.12 \pm 0.08$ & $0.70 \pm 0.05$ & 3.540 .17 & $0.95(45)$ & $2.85 / 2.25$ \\
\hline $94150-03-01-00$ & 55170.34 & $0.97 \pm 0.09$ & $0.24 \pm 0.08$ & $0.73 \pm 0.02$ & 12.950 .19 & $1.06(45)$ & $11.14 / 7.74$ \\
\hline $94150-03-01-01$ & 55173.36 & $0.97 \pm 0.08$ & $0.24 \pm 0.07$ & $0.73 \pm 0.04$ & 11.860 .18 & $1.00(45)$ & $10.18 / 7.07$ \\
\hline 9415 & & & $0.23 \pm$ & & & & $8.99 / 6.33$ \\
\hline $94150-03-01-03$ & 55175.96 & $0.95 \pm 0.07$ & $0.25 \pm 0.06$ & $0.73 \pm 0.03$ & 7.950 .12 & $1.12(45)$ & $6.85 / 4.73$ \\
\hline 94150-03-01-04 & 55175.19 & $0.98 \pm 0.05$ & $0.25 \pm 0.05$ & $0.74 \pm 0.02$ & 10.450 .13 & $1.13(45)$ & $8.97 / 6.25$ \\
\hline $94150-03-02-00$ & 55176.44 & $0.94 \pm 0.06$ & $0.28 \pm 0.04$ & $0.75 \pm 0.03$ & 7.230 .19 & $1.07(45)$ & $6.31 / 4.28$ \\
\hline $94150-03-02-01$ & 55177.27 & $0.93 \pm 0.08$ & $0.25 \pm 0.09$ & $0.81 \pm 0.03$ & 5.850 .17 & $0.94(45)$ & $5.01 / 3.56$ \\
\hline $94150-03-02-02$ & 55178.19 & $0.95 \pm 0.07$ & $0.25 \pm 0.08$ & $0.82 \pm 0.04$ & 6.010 .13 & $1.09(45)$ & $5.12 / 3.67$ \\
\hline $94150-03-02-03$ & 55179.17 & $0.95 \pm 0.07$ & $0.25 \pm 0.08$ & $0.82 \pm 0.04$ & 6.290 .17 & $1.02(45)$ & $5.38 / 3.83$ \\
\hline $94150-03-02-04$ & 55180.15 & $0.95 \pm 0.07$ & $0.26 \pm 0.08$ & $0.83 \pm 0.04$ & 6.350 .14 & $1.04(45)$ & $5.51 / 3.86$ \\
\hline $94150-03-02-05$ & 55181.19 & $0.96 \pm 0.08$ & $0.27 \pm 0.08$ & $0.83 \pm 0.02$ & 6.420 .18 & $1.06(45)$ & $5.57 / 3.91$ \\
\hline $94150-03-02-06$ & 55182.76 & & & & & $0.97(45)$ & $6.47 / 4.35$ \\
\hline $95149-18-01-02$ & 55293.02 & $0.98 \pm 0.04$ & $0.32 \pm 0.05$ & $0.78 \pm 0.04$ & 7.410 .13 & $0.91(45)$ & $6.61 / 4.40$ \\
\hline $95149-18-01-03$ & 55293.38 & $0.97 \pm 0.05$ & $0.32 \pm 0.09$ & $0.78 \pm 0.05$ & 7.010 .12 & $0.97(45)$ & $6.26 / 4.16$ \\
\hline $95149-18-01-05$ & 55294.28 & $0.98 \pm 0.06$ & $0.26 \pm 0.08$ & $0.80 \pm 0.06$ & 8.520 .11 & $0.98(45)$ & $7.43 / 5.15$ \\
\hline $95149-18-01-08$ & 55293.15 & $0.98 \pm 0.07$ & $0.28 \pm 0.09$ & $0.80 \pm 0.05$ & 7.170 .15 & $0.96(45)$ & $6.30 / 4.31$ \\
\hline $95149-18-01-09$ & 55292.89 & $0.99 \pm 0.04$ & $0.28 \pm 0.05$ & $0.80 \pm 0.06$ & 7.680 .17 & $0.98(45)$ & $6.74 / 4.62$ \\
\hline $95149-18-02-00$ & 55295.77 & $0.98 \pm 0.05$ & $0.29 \pm 0.08$ & $0.78 \pm 0.02$ & 8.020 .15 & $0.94(45)$ & $7.13 / 4.78$ \\
\hline $95149-18-02-01$ & 55296.83 & $0.98 \pm 0.05$ & $0.31 \pm 0.09$ & $0.77 \pm 0.06$ & 6.750 .13 & $0.96(45)$ & $6.06 / 4.00$ \\
\hline $95149-18-02-02$ & 55297.29 & $0.97 \pm 0.08$ & $0.33 \pm 0.07$ & $0.76 \pm 0.04$ & 6.530 .19 & $0.97(45)$ & $5.92 / 3.84$ \\
\hline
\end{tabular}

Notes. Parameter errors correspond to $90 \%$ confidence level. The spectral model is tbabs*bmc; where $N_{\mathrm{H}}$ is fixed at a value $5.0 \times 10^{22} \mathrm{~cm}^{-2}$ (see Sect. 3.2.1). ${ }^{(\dagger)}$ When parameter $\log (A) \gg 1$, this parameter is fixed at 2.0 (see comments in the text). ${ }^{(\dagger \dagger)}$ For normalization parameter $N_{\text {BMC }}=L_{35} / d_{10}^{2}$ (see Eqs. (3) and (B.2)) where $L_{35}$ is the source luminosity in units of $10^{35} \mathrm{erg} \mathrm{s}^{-1}$ and $d_{10}$ is the distance to the source in units of $10 \mathrm{kpc}{ }^{(\dagger \dagger)}$ Spectral fluxes $\left(F_{1} / F_{2}\right)$ in units of $\times 10^{-9} \mathrm{ergs} \mathrm{s}^{-1} \mathrm{~cm}^{-2}$ for (3-10) and (10-20) keV energy ranges, respectively. 
Table B.3. Best-fit parameters of spectral analysis of RXTE observations of M 87 ( $R 1-R 2$ sets) in 3-50 keV energy range.

\begin{tabular}{|c|c|c|c|c|c|c|c|c|c|}
\hline Observational ID & $\begin{array}{l}\text { MJD } \\
\text { (day) }\end{array}$ & $\alpha=\Gamma-1$ & $\begin{array}{l}N_{\mathrm{BMC}}{ }^{(\dagger)} \\
\left(L_{37} / d_{10}^{2}\right)\end{array}$ & $\begin{array}{c}E_{\text {cut }} \\
(\mathrm{keV})\end{array}$ & $\begin{array}{c}E_{\text {fold }} \\
(\mathrm{keV})\end{array}$ & $\begin{array}{c}E_{\text {line }} \\
(\mathrm{keV})\end{array}$ & $\begin{array}{c}\sigma_{\text {line }} \\
(\mathrm{keV})\end{array}$ & $N_{\text {line }}^{(\dagger)}$ & $\chi_{\text {red }}^{2}$ (d.o.f.) \\
\hline $30216-01-01-000$ & 50812.94 & $2.02 \pm 0.06$ & $1.18 \pm 0.08$ & $3.6 \pm 0.1$ & $9.9 \pm 0.8$ & $6.4 \pm 0.1$ & $0.7 \pm 0.2$ & $0.10 \pm 0.01$ & $0.95(40)$ \\
\hline $30216-01-01-010$ & 50813.74 & $2.04 \pm 0.02$ & $1.58 \pm 0.01$ & $7.3 \pm 0.9$ & $4.7 \pm 0.7$ & $6.6 \pm 0.1$ & $0.2 \pm 0.1$ & $0.09 \pm 0.02$ & $0.95(40)$ \\
\hline 30216-01-01-02 & 50814.74 & $2.00 \pm 0.01$ & $1.11 \pm 0.01$ & $6.7 \pm 0.3$ & $4.2 \pm 0.3$ & $6.5 \pm 0.2$ & $0.5 \pm 0.2$ & $0.08 \pm 0.01$ & $0.93(40)$ \\
\hline $30216-01-02-00$ & 50822.61 & $2.01 \pm 0.05$ & $1.13 \pm 0.02$ & $7.0 \pm 0.3$ & $3.6 \pm 0.2$ & $6.4 \pm 0.3$ & $0.4 \pm 0.1$ & $0.07 \pm 0.01$ & $0.98(40)$ \\
\hline $30216-01-02-01$ & 50823.56 & $1.99 \pm 0.06$ & $1.14 \pm 0.02$ & $7.2 \pm 0.4$ & $3.4 \pm 0.3$ & $6.5 \pm 0.1$ & $0.3 \pm 0.1$ & $0.09 \pm 0.01$ & $0.86(40)$ \\
\hline $30216-01-02-020$ & 50824.62 & $2.00 \pm 0.05$ & $1.12 \pm 0.06$ & $6.8 \pm 0.3$ & $3.8 \pm 0.3$ & $6.3 \pm 0.2$ & $0.4 \pm 0.1$ & $0.10 \pm 0.03$ & $0.93(40)$ \\
\hline 30216-01-03-00 & 50832.95 & $2.02 \pm 0.07$ & $1.14 \pm 0.07$ & $7.0 \pm 0.4$ & $3.6 \pm 0.3$ & $6.4 \pm 0.2$ & $0.5 \pm 0.2$ & $0.10 \pm 0.02$ & $0.86(40)$ \\
\hline 30216-01-03-01 & 50833.68 & $2.01 \pm 0.05$ & $1.13 \pm 0.07$ & $6.3 \pm 0.5$ & $5.1 \pm 0.6$ & $6.5 \pm 0.1$ & $0.4 \pm 0.1$ & $0.08 \pm 0.01$ & $0.99(40)$ \\
\hline $30216-01-03-02$ & 50833.97 & $2.00 \pm 0.08$ & $1.15 \pm 0.08$ & $6.7 \pm 0.4$ & $4.2 \pm 0.4$ & $6.5 \pm 0.2$ & $0.4 \pm 0.1$ & $0.09 \pm 0.01$ & $0.96(40)$ \\
\hline 30216-01-03-03 & 50833.29 & $2.00 \pm 0.05$ & $1.14 \pm 0.08$ & $6.2 \pm 0.6$ & $5.2 \pm 0.8$ & $6.3 \pm 0.3$ & $0.7 \pm 0.2$ & $0.10 \pm 0.04$ & $0.95(40)$ \\
\hline 30216-01-03-04 & 50835.56 & $2.03 \pm 0.05$ & $1.19 \pm 0.09$ & $4.6 \pm 0.9$ & $7.2 \pm 0.9$ & $6.5 \pm 0.1$ & $0.4 \pm 0.1$ & $0.10 \pm 0.03$ & $0.98(40)$ \\
\hline $30216-01-04-00$ & 50843.61 & $2.02 \pm 0.06$ & $1.17 \pm 0.09$ & $6.5 \pm 0.8$ & $4.5 \pm 0.5$ & $6.5 \pm 0.2$ & $0.4 \pm 0.1$ & $0.08 \pm 0.02$ & $0.97(40)$ \\
\hline $30216-01-04-01$ & 50844.70 & $2.01 \pm 0.08$ & $1.16 \pm 0.08$ & $6.8 \pm 0.4$ & $4.2 \pm 0.3$ & $6.4 \pm 0.2$ & $0.4 \pm 0.1$ & $0.10 \pm 0.01$ & $0.99(40)$ \\
\hline 30216-01-04-02 & 50845.77 & $2.08 \pm 0.09$ & $1.29 \pm 0.06$ & $7.1 \pm 0.9$ & $4.7 \pm 0.9$ & $6.3 \pm 0.2$ & $0.4 \pm 0.1$ & $0.10 \pm 0.01$ & $0.96(40)$ \\
\hline $30216-01-04-03$ & 50843.89 & $2.09 \pm 0.08$ & $1.31 \pm 0.07$ & $7.3 \pm 1.2$ & $4.5 \pm 0.9$ & $6.5 \pm 0.1$ & $0.6 \pm 0.2$ & $0.08 \pm 0.01$ & $0.98(40)$ \\
\hline 30216-01-04-04 & 50846.55 & $2.05 \pm 0.09$ & $1.79 \pm 0.09$ & $7.1 \pm 1.8$ & $7.8 \pm 1.6$ & $6.4 \pm 0.3$ & $0.4 \pm 0.1$ & $0.09 \pm 0.03$ & $0.97(40)$ \\
\hline $30216-01-04-05$ & 50846.88 & $1.98 \pm 0.08$ & $1.27 \pm 0.06$ & $6.9 \pm 1.0$ & $5.0 \pm 1.8$ & $6.5 \pm 0.2$ & $0.7 \pm 0.2$ & $0.10 \pm 0.01$ & $0.94(40)$ \\
\hline $95145-01-01-00$ & 55296.79 & $0.99 \pm 0.08$ & $0.37 \pm 0.09$ & $2.8 \pm 1.0$ & $3.7 \pm 0.3$ & $6.3 \pm 0.2$ & $0.4 \pm 0.1$ & $0.10 \pm 0.08$ & $0.93(40)$ \\
\hline 95145-01-01-01 & 55298.94 & $0.86 \pm 0.07$ & $0.34 \pm 0.07$ & $3.3 \pm 1.4$ & $3.4 \pm 0.2$ & $6.5 \pm 0.1$ & $0.5 \pm 0.1$ & $0.09 \pm 0.02$ & $0.97(40)$ \\
\hline 95145-01-01-02 & 55299.99 & $0.8 \pm 0.1$ & $0.33 \pm 0.09$ & $2.9 \pm 1.5$ & $3.1 \pm 1.3$ & $6.4 \pm 0.2$ & $0.6 \pm 0.2$ & $0.09 \pm 0.02$ & $1.03(40)$ \\
\hline 95145-01-01-03 & 55301.00 & $0.76 \pm 0.09$ & $0.32 \pm 0.08$ & $3.1 \pm 1.8$ & $3.0 \pm 1.8$ & $6.5 \pm 0.2$ & $0.4 \pm 0.1$ & $0.10 \pm 0.06$ & $0.95(40)$ \\
\hline
\end{tabular}

Notes. Parameter errors correspond to $90 \%$ confidence level. The spectral model is tbabs*bmc*highecut; where $N_{\mathrm{H}}$ is fixed at a value $1.94 \times 10^{20} \mathrm{~cm}^{-2}$ (see Sect. 3.2.1) and the parameter $k T_{\mathrm{s}}$ of the BMC model was fixed at $0.4 \mathrm{keV}$ parameter $\log (A)$ is low variable around 2.0 .

${ }^{(\dagger)}$ For normalization parameter $L_{37}$ is the source luminosity in units of $10^{37} \mathrm{erg} \mathrm{s}^{-1}$ and $d_{10}$ is the distance to the source in units of $10 \mathrm{kpc}$; and the Gaussian component is in units of $10^{-2} \times$ total photons $\mathrm{cm}^{-2} \mathrm{~s}^{-1}$ in line. 